\title{
On LA-Courant Algebroids and Poisson Lie 2-Algebroids
}

\section{Jotz Lean ${ }^{1}$ iD}

Received: 13 March 2020 / Accepted: 28 July 2020 / Published online: 20 August 2020

(C) The Author(s) 2020

\begin{abstract}
This paper reformulates Li-Bland's definition for LA-Courant algebroids, or Poisson Lie 2-algebroids, in terms of split Lie 2-algebroids and self-dual 2-representations. This definition generalises in a precise sense the characterisation of (decomposed) double Lie algebroids via matched pairs of 2-representations. We use the known geometric examples of LA-Courant algebroids in order to provide new examples of Poisson Lie 2-algebroids, and we explain in this general context Roytenberg's equivalence of Courant algebroids with symplectic Lie 2-algebroids. We study further the core of an LA-Courant algebroid and we prove that it carries an induced degenerate Courant algebroid structure. In the nondegenerate case, this gives a new construction of a Courant algebroid from the corresponding symplectic Lie 2-algebroid. Finally we completely characterise VB-Dirac and LA-Dirac structures via simpler objects, that we compare to Li-Bland's pseudo-Dirac structures.
\end{abstract}

Keywords Positively graded manifolds · Courant algebroids · Lie algebroids . Poisson and symplectic structures · Double vector bundles ·

Representations up to homotopy

Mathematics Subject Classification (2010) Primary 58A50 $\cdot$ 17B63 · 53D17

Secondary $70 \mathrm{G} 45 \cdot 53 \mathrm{~B} 05 \cdot 58 \mathrm{H} 99$

\section{Introduction}

In the early nineties Courant algebroids appeared in Poisson geometry as a convenient framework for the study of constrained Hamiltonian systems [1]. They are now also at the base of the definition of generalised complex geometry [6, 7]. The graded geometric description of Courant algebroids as symplectic Lie 2-algebroids are due to

M. Jotz Lean

madeleine.jotz-lean@mathematik.uni-goettingen.de

1 Mathematisches Institut, Georg-August-Universität Göttingen, Göttingen, Germany 
Roytenberg [25] and Severa [26]. This description is the key to the attempts made so far to find the global geometric objects 'integrating' Courant algebroids (see e.g. [16, $21,22,27,28])$. Our goal in $[10,12]$ and this paper is to explain Roytenberg and Severa's description from the more general point of view of VB-Courant algebroids versus Lie 2-algebroids. We show that the symplectic structure of the symplectic Lie 2-algebroid that corresponds to a Courant algebroid could rather be understood as a structure of tangent space, just like the canonical symplectic structure on a cotangent bundle $T^{*} M$ is just the dual Poisson structure to the standard tangent Lie algebroid $T M \rightarrow M$, which happens to be nondegenerate. In order to do that, our main goal here is to understand in detail the equivalence between Poisson Lie 2-algebroids and LA-Courant algebroids [14].

This paper surveys the author's recent equivalences of graded manifolds of degree 2 with metric double vector bundles [10] (see also [2]), of self-dual 2-representations with decomposed metric VB-algebroids [10] (using [4]), and of split Lie 2-algebroids with decomposed VB-Courant algebroids $[12,14]$. We combine all those results to provide an alternative definition for Li-Bland's LA-Courant algebroids, which are equivalent to Poisson Lie 2-algebroids [14]; i.e. Lie 2-algebroids endowed with a Poisson structure of degree -2 that makes the homological vector field a Poisson vector field.

We prove on the one hand that a split Poisson Lie 2-algebroid is equivalent to the matched pair of a self-dual 2-representation with a split Lie 2-algebroid. Here, the self-dual 2-representation is the one that is equivalent to the Poisson structure of degree -2 .

On the other hand, given a Poisson Lie 2-algebroid, a splitting of the underlying [2]-manifold is equivalent to a decomposition of the corresponding metric double vector bundle [10]. The underlying split Lie 2-algebroid is then equivalent to a VB-Courant algebroid structure in this decomposition [12] and the underlying split Poisson structure of degree -2 is equivalent to the decomposition of a (metric) VB-algebroid structure on the other side of the metric double vector bundle [10]. The compatibility of the graded Poisson structure and the Lie 2-algebroid structure is equivalent to the VB-Courant algebroid and the metric VB-algebroid defining together an LA-Courant algebroid (short for Lie algebroid Courant algebroid) [14]. Hence, since LA-Courant algebroids are equivalent to Poisson Lie 2-algebroids [14], we find that a metric double vector bundle with a VB-Courant algebroid structure and a metric VB-algebroid structure define together an LA-Courant algebroid if and only if, in any decomposition, the induced split Lie 2-algebroid and the induced self-dual 2-representation build a matched pair.

The original definition of an LA-Courant algebroid in [14] is inspired from and as technical as Mackenzie's first definition of a double Lie algebroid [20]. In short, a VB-Courant algebroid with a linear Lie algebroid on its other side is an LA-Courant algebroid if a relation defined by the Lie algebroid structure in the tangent prolongation of the VB-Courant algebroid-the underlying geometric structure is a triple vector bundle-is a Dirac structure in this tangent prolongation. In an earlier version of this work [8], we deduced from this definition our equations in the definition of the matched pair of a split Lie 2-algebroid with a self-dual 2-representation. Then we 
found easily that those equations were also equivalent to the homological vector field defining the Lie 2-algebroid to be a Poisson vector field.

However, working out the equations directly from Li-Bland's LA-Courant algebroid condition is very long and technical (see the Appendix B of [8]). In this paper, we prefer therefore using Li-Bland's equivalence [14] in order to prove our characterisation of an LA-Courant algebroid via the matched pair. We complement therefore, with explicit equations, Li-Bland's definition of LA-Courant algebroids. We explain again along the way the parallels between the theory of Lie algebroids, double Lie algebroids and 2-representations on the one hand, and Courant algebroids, LA-Courant algebroids and Lie 2-algebroids on the other hand. We find in particular that a matched pair of 2-representations [5] not only defines a split Lie 2-algebroid [12], but also a split Poisson Lie 2-algebroid - this is in general a different construction. Note here that the five equations that we find show that in the chosen splitting of the underlying Lie 2-algebroid, the Poisson structure defines a morphism from the coadjoint to the adjoint representation up to homotopy of the Lie 2-algebroid [13].

We prove further that the core of an LA-Courant algebroid has an induced degenerate Courant algebroid structure-just like the core of a double Lie algebroid has an induced Lie algebroid structure [5, 20]. This allows us to find a new constructive manner to define the Courant algebroid corresponding to a given symplectic Lie 2-algebroid [25].

Finally, we study VB- and LA-Dirac structures in VB- and LA-Courant algebroids, and in particular, we prove that LA-Dirac structures are double Lie algebroids. More precisely, any double Lie algebroid can be understood as an LA-Dirac structure in an appropriate LA-Courant algebroid; just like any Lie algebroid can be seen as a Dirac structure in the induced Courant algebroid. This shows that the seven equations defining a matched pair of 2-representations [5] can in fact be deduced from the 5 equations defining the matched pair of a split Lie 2-algebroid with a self-dual 2-representation. For completeness, we explain as well how LiBland's pseudo-Dirac structures [15] fit in our description of LA-Dirac structures in the tangent prolongation of a Courant algebroid. Finally, we show how a special Manin pair is associated to any LA-Dirac structure; the study of the geometric meaning of this Manin pair will be the subject of future investigations by the author.

Outline of the Paper In Section 2, we recall some general notions and conventions around dull brackets, Dorfman connections, Courant algebroids, graded manifolds and double Lie algebroids. In Section 2.5 we recall the equivalence of metric double vector bundles with [2]-manifolds, of metric VB-algebroids with Poisson [2]manifolds and with self-dual 2-representations when decomposed. We recall also the equivalence of decompositions of VB-Courant algebroids with split Lie 2-algebroids. In Section 3 we give the definition of the matched pair of a split Lie 2-algebroid with a self-dual 2-representation, and we prove our main theorem, giving a new definition of an LA-Courant algebroid. In Section 4 we study the core of an LACourant algebroid and in Section 5 we describe VB- and LA-Dirac structures in 
decompositions. The text is illustrated with several examples that were prepared in $[9,12]$.

\section{Prerequisites, Notation and Conventions}

We recall in this section some necessary background, and we set our notation convention.

\subsection{General Conventions}

We write $p_{M}: T M \rightarrow M, q_{E}: E \rightarrow M$ for vector bundle maps. For a vector bundle $Q \rightarrow M$ we often identify without further mentioning the vector bundle $\left(Q^{*}\right)^{*}$ with $Q$ via the canonical isomorphism. We write $\langle\cdot, \cdot\rangle$ for the canonical pairing of a vector bundle with its dual; i.e. $\left\langle q_{m}, \tau_{m}\right\rangle=\tau_{m}\left(q_{m}\right)$ for $q_{m} \in Q$ and $\tau_{m} \in Q^{*}$. We use several different pairings; in general, which pairing is used is clear from its arguments. Given a section $\varepsilon$ of $E^{*}$, we always write $\ell_{\varepsilon}: E \rightarrow \mathbb{R}$ for the linear function associated to it, i.e. the function defined by $e_{m} \mapsto\left\langle\varepsilon(m), e_{m}\right\rangle$ for all $e_{m} \in E$.

Let $M$ be a smooth manifold. We denote by $\mathfrak{X}(M)$ and $\Omega^{1}(M)$ the sheaves of local smooth sections of the tangent and the cotangent bundle, respectively. For an arbitrary vector bundle $E \rightarrow M$, the sheaf of local sections of $E$ will be written $\Gamma(E)$.

\subsection{Dull Brackets, Dorfman Connections, Courant Algebroids}

Let $q_{Q}: Q \rightarrow M$ be an anchored vector bundle, with anchor $\rho_{Q}: Q \rightarrow T M$. A dull bracket on sections of $Q$ is here an $\mathbb{R}$-bilinear, skew-symmetric bracket $\llbracket \cdot, \cdot \rrbracket: \Gamma(Q) \times \Gamma(Q) \rightarrow \Gamma(Q)$ that is compatible with the anchor, $\rho_{Q} \llbracket q_{1}, q_{2} \rrbracket=$ $\left[\rho_{Q}\left(q_{1}\right), \rho_{Q}\left(q_{2}\right)\right]$ and that satisfies the Leibniz identity

$$
\llbracket q_{1}, f q_{2} \rrbracket=\rho_{Q}\left(q_{1}\right)(f) q_{2}+f \llbracket q_{1}, q_{2} \rrbracket
$$

for all $f \in C^{\infty}(M)$ and $q_{1}, q_{2} \in \Gamma(Q)$.

Dualising the dull bracket in the sense of derivations, we get a Dorfman connection $\Delta: \Gamma(Q) \times \Gamma\left(Q^{*}\right) \rightarrow \Gamma\left(Q^{*}\right)$ :

$$
\rho_{Q}(q)\left\langle q^{\prime}, \tau\right\rangle=\left\langle\llbracket q, q^{\prime} \rrbracket, \tau\right\rangle+\left\langle q^{\prime}, \Delta_{q} \tau\right\rangle
$$

for all $q, q^{\prime} \in \Gamma(Q)$ and $\tau \in \Gamma\left(Q^{*}\right)$. The Leibniz identities in the two entries of the dull bracket give $\Delta_{q}(f \tau)=f \Delta_{q} \tau+\rho_{Q}(q)(f) \tau$ and $\Delta_{f q} \tau=f \Delta_{q} \tau+\langle q, \tau\rangle \rho_{Q}^{*} \mathbf{d} f$ for $q \in \Gamma(Q), \tau \in \Gamma\left(Q^{*}\right)$ and $f \in C^{\infty}(M)$. The compatibility of the bracket with the anchor reads

$$
\Delta_{q}\left(\rho_{Q}^{*} \mathbf{d} f\right)=\rho_{Q}^{*} \mathbf{d}\left(\rho_{Q}(q)(f)\right)
$$

for $q \in \Gamma(Q)$ and $f \in C^{\infty}(M)$. 
Given an $\mathbb{R}$-bilinear skew-symmetric bracket $[\cdot, \cdot]$ on sections of a vector bundle $E \rightarrow M$, its Jacobiator is the map $\mathrm{Jac}_{[\cdot, \cdot]}: \Gamma(E) \times \Gamma(E) \times \Gamma(E) \rightarrow \Gamma(E)$,

$$
\operatorname{Jac}_{[\cdot, \cdot]}\left(e_{1}, e_{2}, e_{3}\right)=\left[\left[e_{1}, e_{2}\right], e_{3}\right]+\left[\left[e_{2}, e_{3}\right], e_{1}\right]+\left[\left[e_{3}, e_{1}\right], e_{2}\right] .
$$

The Jacobiator of a dull bracket satisfies

$$
\operatorname{Jac}_{\llbracket \cdot, \cdot \rrbracket}\left(q_{1}, q_{2}, q_{3}\right)=R_{\Delta}\left(q_{1}, q_{2}\right)^{*} q_{3},
$$

where $R_{\Delta} \in \Omega^{2}\left(Q, \operatorname{End}\left(Q^{*}\right)\right)$ is the curvature of the dual Dorfman connection: $R_{\Delta}\left(q_{1}, q_{2}\right) \tau=\Delta_{q_{1}} \Delta_{q_{2}} \tau-\Delta_{q_{2}} \Delta_{q_{1}} \tau-\Delta_{\llbracket q_{1}, q_{2} \rrbracket} \tau$.

A dull algebroid $\left(Q, \rho_{Q}, \llbracket \cdot, \cdot \rrbracket\right)$ as above defines as usual a Koszul differential operator $d_{Q}: \Omega^{\bullet}(Q) \rightarrow \Omega^{\bullet+1}(Q)$ with $\mathbf{d}_{Q}(\omega \wedge \eta)=\mathbf{d}_{Q} \omega \wedge \eta+(-1)^{|\eta|} \omega \wedge \mathbf{d}_{Q} \eta$ for all $\omega, \eta \in \Omega^{\bullet}(Q)$.

Let $E$ be a vector bundle over the same base manifold $M$. A linear $Q$-connection on $E$ is a linear connection $\nabla: \Gamma(Q) \times \Gamma(E) \rightarrow \Gamma(E)$. The curvature $R_{\nabla} \in$ $\Omega^{2}(Q, \operatorname{End}(E))$ of $\nabla$ is defined as usual. The dull bracket and the connection define together a differential operator $\mathbf{d}_{\nabla}: \Omega^{\bullet}(Q, E) \rightarrow \Omega^{\bullet+1}(Q, E): \mathbf{d}_{\nabla} e=\nabla . e$ for $e \in \Gamma(E)$, and $\mathbf{d}_{\nabla}(\omega \wedge \eta)=\mathbf{d}_{Q} \omega \wedge \eta+(-1)^{|\eta|} \omega \wedge \mathbf{d}_{\nabla} \eta$ for $\omega \in \Omega^{\bullet}(Q)$ and $\eta \in \Omega^{\bullet}(Q, E)$.

Consider an anchored vector bundle $(\mathrm{E} \rightarrow M, \rho)$. Assume that $\mathrm{E}$ is paired with itself via a pairing $\langle\cdot, \cdot\rangle: \mathrm{E} \times_{M} \mathrm{E} \rightarrow \mathbb{R}$ and that there exists a map $\mathcal{D}: C^{\infty}(M) \rightarrow$ $\Gamma(\mathrm{E})$ such that $\langle\mathcal{D} f, e\rangle=\rho(e)(f)$ for all $f \in C^{\infty}(M)$. Then $\mathrm{E} \rightarrow M$ is a degenerate Courant algebroid over the manifold $M$ if $E$ is in addition equipped with an $\mathbb{R}$-bilinear bracket $\llbracket \cdot, \cdot \rrbracket$ on the smooth sections $\Gamma(E)$ such that the following conditions are satisfied:

(CA1) $\llbracket e_{1}, \llbracket e_{2}, e_{3} \rrbracket \rrbracket=\llbracket \llbracket e_{1}, e_{2} \rrbracket, e_{3} \rrbracket+\llbracket e_{2}, \llbracket e_{1}, e_{3} \rrbracket \rrbracket$,

(CA2) $\rho\left(e_{1}\right)\left\langle e_{2}, e_{3}\right\rangle=\left\langle\llbracket e_{1}, e_{2} \rrbracket, e_{3}\right\rangle+\left\langle e_{2}, \llbracket e_{1}, e_{3} \rrbracket\right\rangle$,

(CA3) $\llbracket e_{1}, e_{2} \rrbracket+\llbracket e_{2}, e_{1} \rrbracket=\mathcal{D}\left\langle e_{1}, e_{2}\right\rangle$,

(CA4) $\rho \llbracket e_{1}, e_{2} \rrbracket=\left[\rho\left(e_{1}\right), \rho\left(e_{2}\right)\right]$,

(CA5) $\llbracket e_{1}, f e_{2} \rrbracket=f \llbracket e_{1}, e_{2} \rrbracket+\left(\rho\left(e_{1}\right) f\right) e_{2}$

for all $e_{1}, e_{2}, e_{3} \in \Gamma(\mathrm{E})$ and $f \in C^{\infty}(M)$. If the pairing $\langle\cdot, \cdot\rangle$ is nondegenerate, then $(\mathrm{E} \rightarrow M, \rho,\langle\cdot, \cdot\rangle, \llbracket \cdot, \cdot \rrbracket)$ is a Courant algebroid $[17,24]$ and Conditions (CA4) and (CA5) follow then from (CA1), (CA2) and (CA3) (see [29] and also [9] for a quicker proof).

\section{$2.3 \mathbb{N}$-Manifolds of Degree 2}

An $\mathbb{N}$-manifold $\mathcal{M}$ of degree 2 and dimension $\left(p ; r_{1}, r_{2}\right)$ is a smooth manifold $M$ of dimension $p$ together a sheaf $C^{\infty}(\mathcal{M})$ of $\mathbb{N}$-graded, graded commutative, associative, unital $C^{\infty}(M)$-algebras over $M$, that is locally freely generated by $r_{1}+r_{2}$ elements $\xi_{1}^{1}, \ldots, \xi_{1}^{r_{1}}, \xi_{2}^{1}, \ldots, \xi_{2}^{r_{2}}$ with $\xi_{i}^{j}$ of degree $i$ for $i=1,2$ and $j \in\left\{1, \ldots, r_{i}\right\}$. We write "[2]-manifold" for "NN-manifold of degree 2". A morphism of $\mathbb{N}$-manifolds 
$\mu: \mathcal{N} \rightarrow \mathcal{M}$ over a smooth map $\mu_{0}: N \rightarrow M$ of the underlying smooth manifolds is a morphism $\mu^{\star}: C^{\infty}(\mathcal{M}) \rightarrow C^{\infty}(\mathcal{N})$ of sheaves of graded algebras over $\mu_{0}^{*}: C^{\infty}(M) \rightarrow C^{\infty}(N)$.

Let $E_{1}$ and $E_{2}$ be smooth vector bundles of finite ranks $r_{1}, r_{2}$ over $M$ and assign the degree $i$ to the fiber coordinates of $E_{i}$, for each $i=1, \ldots, n$. The direct sum $E=E_{1} \oplus E_{2}$ is a graded vector bundle with grading concentrated in degrees 1 and 2 . The [2]-manifold $E_{1}[-1] \oplus E_{2}[-2]$ has the elements of local frames of $E_{i}^{*}$ as local generators of degree $i$, for $i=1,2$, and so dimension $\left(p ; r_{1}, r_{2}\right)$. A [2]-manifold $\mathcal{M}=E_{1}[-1] \oplus E_{2}[-2]$ defined in this manner by a graded vector bundle is called a split [2]-manifold. In other words, we have $C^{\infty}(\mathcal{M})^{0}=C^{\infty}(M), C^{\infty}(\mathcal{M})^{1}=$ $\Gamma\left(E_{1}^{*}\right)$ and $C^{\infty}(\mathcal{M})^{2}=\Gamma\left(E_{2}^{*} \oplus \wedge^{2} E_{1}^{*}\right)$. A morphism $\mu: F_{1}[-1] \oplus F_{2}[-2] \rightarrow$ $E_{1}[-1] \oplus E_{2}[-2]$ of split [2]-manifolds over the bases $M$ and $N$, respectively, consists of a smooth map $\mu_{0}: N \rightarrow M$, three vector bundle morphisms $\mu_{1}: F_{1} \rightarrow E_{1}$, $\mu_{2}: F_{2} \rightarrow E_{2}$ and $\mu_{12}: \wedge^{2} F_{1} \rightarrow E_{2}$ over $\mu_{0}$. The map $\mu^{\star}$ sends a degree 1 function $\xi \in \Gamma\left(E_{1}^{*}\right)$ to $^{1} \mu_{1}^{\star} \xi \in \Gamma\left(F_{1}^{*}\right)$ and a degree 2-function $\xi \in \Gamma\left(E_{2}^{*}\right)$ to $\mu_{2}^{\star} \xi+\mu_{12}^{\star} \xi \in \Gamma\left(F_{2}^{*} \oplus \wedge^{2} F_{1}^{*}\right)$.

\subsection{Double Lie Algebroids and Matched Pairs of 2-Representations}

We refer to Section 2.2 of [10] for the definition of a double vector bundle, and for the necessary background on their linear and core sections, and on their linear splittings and dualisations. Sections 2.3-2.5 of [10] recall the definition of a VBalgebroid, and also the equivalence of 2-term representations up to homotopy (called here "2-representations" for short) with linear decompositions of VB-algebroids [4]. The notation that we use here is the same as in [10]. Of course, we also refer to [4, 19,23 ] for more details on double vector bundles and VB-algebroids.

In this section we only recall the correspondence of decompositions of double Lie algebroids with matched pairs of 2-representations.

If $(D, A, B, M)$ is a VB-algebroid with Lie algebroid structures on $D \rightarrow B$ and $A \rightarrow M$, then the dual vector bundle $D_{B}^{*} \rightarrow B$ has a Lie-Poisson structure (a linear Poisson structure), and the structure on $D_{B}^{*}$ is also Lie-Poisson with respect to $D_{B}^{*} \rightarrow C^{*}[20,3.4]$. Dualising this bundle gives a Lie algebroid structure on $\left(D_{B}^{*}\right)_{C^{*}}^{*} \rightarrow C^{*}$. This equips the double vector bundle $\left(\left(D_{B}^{*}\right)_{C^{*}}^{*} ; C^{*}, A ; M\right)$ with a VB-algebroid structure. Using the isomorphism defined by the non-degenerate pairing $-\langle\cdot, \cdot\rangle: D_{A}^{*} \times C^{*} D_{B}^{*} \rightarrow \mathbb{R}$, (see [19] and [10, Section 2.2.4] for a summary and our sign convention), the double vector bundle $\left(D_{A}^{*} \rightarrow C^{*} ; A \rightarrow M\right)$ is also a VBalgebroid. In the same manner, if $(D \rightarrow A, B \rightarrow M)$ is a VB-algebroid then we use the non-degenerate pairing $\langle\cdot\rangle:, D_{A}^{*} \times C^{*} D_{B}^{*} \rightarrow \mathbb{R}$ to get a VB-algebroid structure on $\left(D_{B}^{*} \rightarrow C^{*} ; B \rightarrow M\right)$.

Let $\Sigma: A \times_{M} B \rightarrow D$ be a linear splitting of $D$ and denote by $\left(\nabla^{B}, \nabla^{C}, R_{A}\right)$ the induced 2-representation of the Lie algebroid $A$ on $\partial_{B}: C \rightarrow B$ (see [4]; this is also recalled in Section 2.5 of [10]). The linear splitting $\Sigma$ induces a linear splitting $\Sigma^{\star}: A \times_{M} C^{*} \rightarrow D_{A}^{*}$ of $D_{A}^{*}$. The 2-representation of $A$ that is associated to this

${ }^{1} \mu_{1}^{\star} \xi \in \Gamma\left(F_{1}^{*}\right)$ is defined by $\left\langle\left(\mu_{1}^{\star} \xi\right)_{p}, e_{p}\right\rangle=\left\langle\xi\left(\mu_{0}(p)\right), \mu_{1}\left(e_{p}\right)\right\rangle$ for all $e_{p} \in F_{1}$. 
splitting is then $\left(\nabla^{C^{*}}, \nabla^{B^{*}},-R_{A}^{*}\right)$ on the complex $\partial_{B}^{*}: B^{*} \rightarrow C^{*}$. This is proved in the appendix of [3].

A double Lie algebroid [20] is a double vector bundle $(D, A, B, M)$ with core $C$, and with Lie algebroid structures on each of $A \rightarrow M, B \rightarrow M, D \rightarrow A$ and $D \rightarrow B$ such that each pair of parallel Lie algebroids gives $D$ the structure of a VBalgebroid, and such that the pair $\left(D_{A}^{*}, D_{B}^{*}\right)$ with the induced Lie algebroid structures on base $C^{*}$ and the pairing $\langle\cdot, \cdot\rangle$, is a Lie bialgebroid.

Consider a double vector bundle $(D ; A, B ; M)$ with core $C$ and a VB-Lie algebroid structure on each of its sides. After the choice of a splitting $\Sigma: A \times_{M} B \rightarrow$ $D$, the Lie algebroid structures on the two sides of $D$ are described by two 2representations [4]. We prove in [5] that $\left(D_{A}^{*}, D_{B}^{*}\right)$ is a Lie bialgebroid over $C^{*}$ if and only if, for any splitting of $D$, the two induced 2-representations form a matched pair as in the following definition [5].

Definition 2.1 Let $\left(A \rightarrow M, \rho_{A},[\cdot, \cdot]\right)$ and $\left(B \rightarrow M, \rho_{B},[\cdot, \cdot]\right)$ be two Lie algebroids and assume that $A$ acts on $\partial_{B}: C \rightarrow B$ up to homotopy via $\left(\nabla^{B}, \nabla^{C}, R_{A}\right)$ and $B$ acts on $\partial_{A}: C \rightarrow A$ up to homotopy via $\left(\nabla^{A}, \nabla^{C}, R_{B}\right){ }^{2}$ Then we say that the two representations up to homotopy form a matched pair if

(m1) $\nabla_{\partial_{A} c_{1}} c_{2}-\nabla_{\partial_{B} c_{2}} c_{1}=-\left(\nabla_{\partial_{A} c_{2}} c_{1}-\nabla_{\partial_{B} c_{1}} c_{2}\right)$,

(m2) $\left[a, \partial_{A} c\right]=\partial_{A}\left(\nabla_{a} c\right)-\nabla_{\partial_{B}} a$,

(m3) $\left[b, \partial_{B} c\right]=\partial_{B}\left(\nabla_{b} c\right)-\nabla_{\partial_{A} c} b$

(m4) $\nabla_{b} \nabla_{a} c-\nabla_{a} \nabla_{b} c-\nabla_{\nabla_{b}} c+\nabla_{\nabla_{a} b} c=R_{B}\left(b, \partial_{B} c\right) a-R_{A}\left(a, \partial_{A} c\right) b$,

(m5) $\partial_{A}\left(R_{A}\left(a_{1}, a_{2}\right) b\right)=-\nabla_{b}\left[a_{1}, a_{2}\right]+\left[\nabla_{b} a_{1}, a_{2}\right]+\left[a_{1}, \nabla_{b} a_{2}\right]+\nabla_{\nabla_{a_{2}} b} a_{1}-$ $\nabla_{\nabla_{a_{1}} b} a_{2}$

(m6) $\partial_{B}\left(R_{B}\left(b_{1}, b_{2}\right) a\right)=-\nabla_{a}\left[b_{1}, b_{2}\right]+\left[\nabla_{a} b_{1}, b_{2}\right]+\left[b_{1}, \nabla_{a} b_{2}\right]+\nabla_{\nabla_{b_{2}} a} b_{1}-$ $\nabla_{\nabla_{b_{1}} a} b_{2}$

for all $a, a_{1}, a_{2} \in \Gamma(A), b, b_{1}, b_{2} \in \Gamma(B)$ and $c, c_{1}, c_{2} \in \Gamma(C)$, and

(m7) $\mathbf{d}_{\nabla^{A}} R_{B}=\mathbf{d}_{\nabla^{B}} R_{A} \in \Omega^{2}\left(A, \wedge^{2} B^{*} \otimes C\right)=\Omega^{2}\left(B, \wedge^{2} A^{*} \otimes C\right)$, where $R_{B}$ is seen as an element of $\Omega^{1}\left(A, \wedge^{2} B^{*} \otimes C\right)$ and $R_{A}$ as an element of $\Omega^{1}\left(B, \wedge^{2} A^{*} \otimes C\right)$.

\subsection{The Equivalence of [2]-Manifolds with Metric Double Vector Bundles}

We begin by summarising the correspondence found in [10] between double vector bundles endowed with a linear metric, and $\mathbb{N}$-manifolds of degree 2 .

A metric double vector bundle is a double vector bundle $(\mathbb{E}, Q, B, M)$ with core $Q^{*}$, equipped with a linear symmetric non-degenerate pairing $\mathbb{E} \times{ }_{B} \mathbb{E} \rightarrow \mathbb{R}$, i.e. such that

(1) $\left\langle\tau_{1}^{\dagger}, \tau_{2}^{\dagger}\right\rangle=0$ for $\tau_{1}, \tau_{2} \in \Gamma\left(Q^{*}\right)$,

(2) $\left\langle\chi, \tau^{\dagger}\right\rangle=q_{B}^{*}\langle q, \tau\rangle$ for $\chi \in \Gamma_{B}^{l}(\mathbb{E})$ linear over $q \in \Gamma(Q)$ and $\tau \in \Gamma\left(Q^{*}\right)$ and

\footnotetext{
${ }^{2}$ For the sake of simplicity, we write in this definition $\nabla$ for all the four connections. It will always be clear from the indexes which connection is meant. We write $\nabla^{A}$ for the $A$-connection induced by $\nabla^{A B}$ and $\nabla^{A C}$ on $\wedge^{2} B^{*} \otimes C$ and $\nabla^{B}$ for the $B$-connection induced on $\wedge^{2} A^{*} \otimes C$.
} 
(3) $\left\langle\chi_{1}, \chi_{2}\right\rangle$ is a linear function on $B$ for $\chi_{1}, \chi_{2} \in \Gamma_{B}^{l}(\mathbb{E})$.

Note that the opposite $(\overline{\mathbb{E}}, Q, B, M)$ of a metric double vector bundle $(\mathbb{E}, B, Q, M)$ is the metric double vector bundle with $\langle\cdot, \cdot\rangle_{\overline{\mathbb{E}}}=-\langle\cdot, \cdot\rangle_{\mathbb{E}}$.

A linear splitting $\Sigma: Q \times_{M} B \rightarrow \mathbb{E}$ is said to be Lagrangian if its image is maximal isotropic in $\mathbb{E} \rightarrow B$. The corresponding horizontal lifts $\sigma_{Q}: \Gamma(Q) \rightarrow \Gamma_{B}^{l}(\mathbb{E})$ and $\sigma_{B}: \Gamma(B) \rightarrow \Gamma_{Q}^{l}(\mathbb{E})$ are then also said to be Lagrangian. By definition, a horizontal lift $\sigma_{Q}: \Gamma(Q) \rightarrow \Gamma_{B}^{l}(\mathbb{E})$ is Lagrangian if and only if $\left\langle\sigma_{Q}\left(q_{1}\right), \sigma_{Q}\left(q_{2}\right)\right\rangle=0$ for all $q_{1}, q_{2} \in \Gamma(Q)$. Showing the existence of a Lagrangian splitting of $\mathbb{E}$ is relatively easy [10]: Note that a general linear decomposition $\Sigma$ of a metric double vector bundle defines as follows a section $\Lambda$ of $S^{2}(Q) \otimes B^{*}$ :

$$
\left\langle\sigma_{Q}\left(q_{1}\right), \sigma_{Q}\left(q_{2}\right)\right\rangle=\ell_{\Lambda\left(q_{1}, q_{2}\right)}
$$

for all $q_{1}, q_{2} \in \Gamma(Q)$. In particular, $\Lambda(q, \cdot): Q \rightarrow B^{*}$ is a morphism of vector bundles for each $q \in \Gamma(Q)$. Define a new horizontal lift $\sigma_{Q}^{\prime}: \Gamma(Q) \rightarrow \Gamma_{B}^{l}(\mathbb{E})$ by

$$
\sigma_{Q}^{\prime}(q)=\sigma_{Q}(q)-\frac{1}{2} \widetilde{\Lambda(q, \cdot)^{*}}
$$

for all $q \in \Gamma(Q)$. It is easy to check that the corresponding linear decomposition $\Sigma^{\prime}$ is Lagrangian.

Further, if $\Sigma^{1}$ and $\Sigma^{2}: Q \times{ }_{M} B \rightarrow \mathbb{E}$ are Lagrangian, then the change of splitting $\phi_{12} \in \Gamma\left(Q^{*} \otimes Q^{*} \otimes B^{*}\right)$ defined by $\Sigma^{2}(q, b)=\Sigma^{1}(q, b)+\widetilde{\phi(q, b)}$ for all $(q, b) \in$ $Q \times{ }_{M} B$, is a section of $Q^{*} \wedge Q^{*} \otimes B^{*}$.

Example 2.2 Let $E \rightarrow M$ be a vector bundle endowed with a symmetric nondegenerate pairing $\langle\cdot, \cdot\rangle: E \times_{M} E \rightarrow \mathbb{R}$ (a metric vector bundle). Then $E \simeq E^{*}$ and the tangent prolongation $T E$ is a metric double vector bundle $(T E, E, T M, M)$ with pairing $T E \times_{T M} T E \rightarrow \mathbb{R}$ the tangent of the pairing $E \times_{M} E \rightarrow \mathbb{R}$. In particular, we have $\left\langle T e_{1}, T e_{2}\right\rangle_{T E}=\ell_{\mathbf{d}\left\langle e_{1}, e_{2}\right\rangle},\left\langle T e_{1}, e_{2}^{\dagger}\right\rangle_{T E}=p_{M}^{*}\left\langle e_{1}, e_{2}\right\rangle$ and $\left\langle e_{1}^{\dagger}, e_{2}^{\dagger}\right\rangle_{T E}=0$ for $e_{1}, e_{2} \in \Gamma(E)$.

Recall from [10, Example 3.11] that linear splittings of $T E$ are equivalent to linear connections $\nabla: \mathfrak{X}(M) \times \Gamma(E) \rightarrow \Gamma(E)$. The Lagrangian splittings of $T E$ are exactly the linear splittings that correspond to metric connections, i.e. linear connections $\nabla: \mathfrak{X}(M) \times \Gamma(E) \rightarrow \Gamma(E)$ that preserve the metric: $\left\langle\nabla \cdot e_{1}, e_{2}\right\rangle+\left\langle e_{1}, \nabla . e_{2}\right\rangle=$ $\mathbf{d}\left\langle e_{1}, e_{2}\right\rangle$ for $e_{1}, e_{2} \in \Gamma(E)$.

Let $(\mathbb{E}, B, Q, M)$ be a metric double vector bundle. Define $\mathcal{C}(\mathbb{E}) \subseteq \Gamma_{Q}^{l}(\mathbb{E})$ as the $C^{\infty}(M)$-submodule of linear sections with isotropic image in $\mathbb{E}$. After the choice of a Lagrangian splitting $\Sigma: Q \times{ }_{M} B \rightarrow \mathbb{E}, \mathcal{C}(\mathbb{E})$ can be written $\mathcal{C}(\mathbb{E}):=\sigma_{B}(\Gamma(B))+\{\tilde{\omega} \mid$ $\left.\omega \in \Gamma\left(Q^{*} \wedge Q^{*}\right)\right\}$. This shows that $\mathcal{C}(\mathbb{E})$ together with $\Gamma_{Q}^{c}(\mathbb{E}) \simeq \Gamma\left(Q^{*}\right)$ span $\mathbb{E}$ as a vector bundle over $Q$. 
We define ${ }^{3}$ a morphism $\Omega: \mathbb{F} \rightarrow \mathbb{E}$ of metric double vector bundles as a pair of maps $\omega^{\star}: \mathcal{C}(\mathbb{E}) \rightarrow \mathcal{C}(\mathbb{F}), \omega_{P}^{\star}: \Gamma\left(Q^{*}\right) \rightarrow \Gamma\left(P^{*}\right)$ together with a smooth map $\omega_{0}: N \rightarrow M$ such that

$$
\begin{aligned}
& \omega^{\star}\left(\widetilde{\tau_{1} \wedge \tau_{2}}\right)=\omega_{P}^{\star} \widetilde{\tau_{1} \wedge \omega_{P}^{\star}} \tau_{2}, \\
& \text { (2) } \omega^{\star}\left(q_{O}^{*} f \cdot \chi\right)=q_{P}^{*}\left(\omega_{0}^{*} f\right) \cdot \omega^{\star}(\chi) \text { and } \\
& \text { (3) } \omega_{P}^{\star}(f \cdot \tau)=\omega_{0}^{*} f \cdot \omega_{P}^{\star} \tau
\end{aligned}
$$

for all $\tau, \tau_{1}, \tau_{2} \in \Gamma\left(Q^{*}\right), f \in C^{\infty}(M)$ and $\chi \in \mathcal{C}(\mathbb{E})$. A morphism $\Omega: Q_{1} \times_{M_{1}}$ $Q_{1} \times M_{1} B_{1}^{*} \rightarrow Q_{2} \times M_{2} Q_{2} \times{ }_{M_{2}} B_{2}^{*}$ of decomposed metric double vector bundles is then described by $\omega_{Q}: Q_{1} \rightarrow Q_{2}, \omega_{B^{*}}: B_{1}^{*} \rightarrow B_{2}^{*}$ and $\omega_{12}: Q_{1} \wedge Q_{1} \rightarrow B_{2}^{*}$, all morphisms of vector bundles over a smooth map $\omega_{0}: M_{1} \rightarrow M_{2}$. For $b \in \Gamma\left(B_{1}\right)$ the isotropic section $b^{l} \in \Gamma_{Q_{2}}^{l}\left(B_{2} \times{ }_{M_{2}} Q_{2} \times_{M_{2}} Q_{2}^{*}\right), b^{l}\left(q_{m}\right)=\left(b(m), q_{m}, 0_{m}^{Q_{2}^{*}}\right)$, is sent by $\omega^{\star}$ to the isotropic section $\left(\omega_{B}^{\star}(b)\right)^{l}+\widetilde{\omega_{12}^{\star}(b)} \in \Gamma_{Q_{1}}^{l}\left(B_{1} \times M_{1} Q_{1} \times{ }_{M_{1}} Q_{1}^{*}\right)$.

We write MDVB for the obtained category of metric double vector bundles. In [10] we established an equivalence between the category of involutive double vector bundles and the category of [2]-manifolds. We also proved there that there is a (contravariant) dualisation equivalence of the categry of involutive double vector bundles with MDVB. This yields the following theorem.

Theorem 2.3 [10] There is a (contravariant) equivalence between the category of [2]-manifolds and the category of metric double vector bundles.

This equivalence establishes in particular an equivalence between split [2]manifold $\mathcal{M}=Q[-1] \oplus B^{*}[-2]$ and the decomposed metric double vector bundle $\left(Q \times_{M} B \times_{M} Q^{*}, B, Q, M\right)$ with the obvious linear metric over $B$.

We quickly describe the functors between the two categories. To construct the geometrisation functor $\mathcal{G}:$ [2]-Man $\rightarrow$ MDVB, take a [2]-manifold and considers its local trivialisations. Changes of local trivialisation define a set of cocycle conditions, that correspond exactly to cocycle conditions for a double vector bundle atlas. The local trivialisations can hence be collated to a double vector bundle, which naturally inherits an involution. See [10] for more details, and remark that this construction is as simple as the construction of a vector bundle from a locally free and finitely generated sheaf of $C^{\infty}(M)$-modules. Conversely, the algebraisation functor $\mathcal{A}$ sends a metric double vector bundle $\mathbb{E}$ to the [2]-manifold defined as follows: the functions of degree 1 are the sections of $\Gamma_{Q}^{c}(\mathbb{E}) \simeq \Gamma\left(Q^{*}\right)$, and the functions of degree 2 are the elements of $\mathcal{C}(\mathbb{E})$. The multiplication of two core sections $\tau_{1}, \tau_{2} \in \Gamma\left(Q^{*}\right)$ is the core-linear section $\widetilde{\tau_{1} \wedge \tau_{2}} \in \mathcal{C}(\mathbb{E})$.

\footnotetext{
${ }^{3}$ A metric double vector bundle ( $\mathbb{E}, B, Q, M$ ) is dual (over $Q$ ) to an involutive double vector bundle [10]. A morphism $\Omega: \mathbb{F} \rightarrow \mathbb{E}$ of metric double vector bundles is defined as a relation $\Omega \subseteq \overline{\mathbb{F}} \times \mathbb{E}$ that is the dual of a morphism of involutive double vector bundles $\omega: \mathbb{F}_{P}^{*} \rightarrow \mathbb{E}_{Q}^{*}[10]$. The characterisation given here is proved in [10].
} 


\subsubsection{Metric VB-Algebroids and Poisson [2]-Manifolds}

The correspondence above of split 2-manifolds with decomposed metric double vector bundles induces a correspondence of split Poisson [2]-manifolds with decomposed metric VB-algebroids. This bijection extends to an equivalence of Poisson [2]-manifolds with metric VB-algebroids [10], but we only need the split objects here.

Consider a metric double vector bundle $(\mathbb{E}, B, Q, M)$ with a linear Lie algebroid on $\mathbb{E} \rightarrow Q$ over a Lie algebroid structure on $B \rightarrow M$. Then $\mathbb{E}$ is a metric VBalgebroid [10] if the bijection
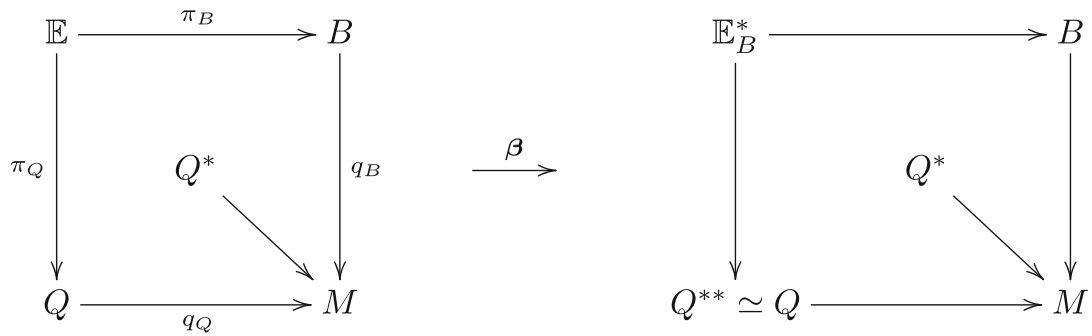

defined by the pairing is a morphism of VB-algebroids, where $\mathbb{E}_{B}^{*} \rightarrow Q^{* *}$ is equipped with the dual Lie algebroid structure to the one on $\mathbb{E} \rightarrow Q$ [10].

Take a decomposed metric double vector bundle $\mathbb{E}=Q \times_{M} B \times_{M} Q^{*}$ with a linear Lie algebroid structure on $Q \times{ }_{M} B \times{ }_{M} Q^{*} \rightarrow Q$ over a Lie algebroid $B \rightarrow M$. Let $\left(\nabla Q, \nabla^{*}, R\right)$ be the corresponding 2-representation of the Lie algebroid $B$ on $\partial_{Q}: Q^{*} \rightarrow Q$. Then $Q \times_{M} B \times_{M} Q^{*}$ is a decomposed metric VB-algebroid if and only if the 2-representation is dual to itself [10]:

$$
\left(\nabla^{Q}\right)^{*}=\nabla^{Q^{*}} \quad \partial_{Q}^{*}=\partial_{Q}, \quad R\left(b_{1}, b_{2}\right)^{*}=-R\left(b_{1}, b_{2}\right) \quad \forall b_{1}, b_{2} \in \Gamma(B) .
$$

That is, the dual decomposed VB-algebroid $\mathbb{E}_{B}^{*}=Q^{* *} \times_{M} B \times_{M} Q^{*} \rightarrow Q^{* *}$ is canonically isomorphic to $\mathbb{E} \rightarrow Q$ via the canonical isomorphism of $Q$ with $Q^{* *}$ [10]. Note that $R\left(b_{1}, b_{2}\right)^{*}=-R\left(b_{1}, b_{2}\right)$ for all $b_{1}, b_{2} \in \Gamma(B)$ is equivalent to $R \in \Omega^{2}\left(B, Q^{*} \wedge Q^{*}\right)$.

Note that a Poisson bracket of degree -2 on a [2]-manifold $\mathcal{M}$ is an $\mathbb{R}$-bilinear map $\{\cdot, \cdot\}: C^{\infty}(\mathcal{M}) \times C^{\infty}(\mathcal{M}) \rightarrow$ $C^{\infty}(\mathcal{M})$ of the graded sheaves of functions, such that $|\{\xi, \eta\}|=|\xi|+|\eta|-2$ for homogeneous elements $\xi, \eta \in C_{\mathcal{M}}^{\infty}(U)$. The bracket is graded skew-symmetric; $\{\xi, \eta\}=-(-1)^{|\xi|}|\eta|\{\eta, \xi\}$ and satisfies the graded Leibniz and Jacobi identities

$$
\left\{\xi_{1}, \xi_{2} \cdot \xi_{3}\right\}=\left\{\xi_{1}, \xi_{2}\right\} \cdot \xi_{3}+(-1)^{\left|\xi_{1}\right|\left|\xi_{2}\right|} \xi_{2} \cdot\left\{\xi_{1}, \xi_{3}\right\}
$$

and

$$
\left\{\xi_{1},\left\{\xi_{2}, \xi_{3}\right\}\right\}=\left\{\left\{\xi_{1}, \xi_{2}\right\}, \xi_{3}\right\}+(-1)^{\left|\xi_{1}\right|\left|\xi_{2}\right|}\left\{\xi_{2},\left\{\xi_{1}, \xi_{3}\right\}\right\}
$$

for homogeneous $\xi_{1}, \xi_{2}, \xi_{3} \in C_{\mathcal{M}}^{\infty}(U)$. A morphism $\mu: \mathcal{N} \rightarrow \mathcal{M}$ of Poisson [2]manifolds satisfies $\mu^{\star}\left\{\xi_{1}, \xi_{2}\right\}=\left\{\mu^{\star} \xi_{1}, \mu^{\star} \xi_{2}\right\}$ for all $\xi_{1}, \xi_{2} \in C_{\mathcal{M}}^{\infty}(U), U$ open in $M$. 
Via the identification of the underlying metric double vector bundle $\mathbb{E}=Q \times_{M}$ $B \times{ }_{M} Q^{*}$ with the [2]-manifold $Q[-1] \oplus B^{*}[-2]$, the metric VB-algebroid structure is equivalent to a degree -2 Poisson structure on $Q[-1] \oplus B^{*}[-2]$ (see [10]):

$$
\begin{aligned}
\left\{f_{1}, f_{2}\right\} & =0, \quad\{\tau, f\}=0, \quad\left\{\tau_{1}, \tau_{2}\right\}=\left\langle\partial_{Q} \tau_{1}, \tau_{2}\right\rangle \\
\{b, f\} & =\rho_{B}(b)(f), \quad\{b, \tau\}=\nabla_{b}^{*} \tau, \quad\left\{b_{1}, b_{2}\right\}=\left[b_{1}, b_{2}\right]-R\left(b_{1}, b_{2}\right) .
\end{aligned}
$$

This identification is compatible with changes of splittings of the [2]-manifolds and changes of decomposition of metric VB-algebroids: The category of Poisson [2]manifolds is equivalent to the category of metric VB-algebroids [10].

Example 2.4 Consider a metric vector bundle $E \rightarrow M$ and a metric connection $\nabla: \mathfrak{X}(M) \times \Gamma(E) \rightarrow \Gamma(E)$. Since $\nabla=\nabla^{*}$ when $E^{*}$ is identified with $E$ via the non-degenerate pairing, the 2-representation $\left(\operatorname{Id}_{E}: E \rightarrow E, \nabla, \nabla, R_{\nabla}\right)$ is self-dual. The metric VB-algebroid structure on $T E \rightarrow E$ is just the standard Lie algebroid structure on the tangent bundle of $E$. See [10] for more details.

\subsubsection{VB-Courant Algebroids and Lie 2-Algebroids}

A VB-Courant algebroid [14] is a metric double vector bundle $(\mathbb{E} \rightarrow B,\langle\cdot, \cdot\rangle)$ with side $Q$ and core $Q^{*}$, together with a linear anchor $\Theta: \mathbb{E} \rightarrow T B$ and a linear Courant algebroid bracket on sections of $\mathbb{E} \rightarrow B$.

A decomposed VB-Courant algebroid can be described as [12] a decomposed metric VB-algebroid $\mathbb{E}=Q \times{ }_{M} B \times{ }_{M} Q^{*} \rightarrow B$ together with an anchor $\rho_{Q}: Q \rightarrow$ $M$, a vector bundle map $\partial_{B}: Q^{*} \rightarrow B$, a dull bracket $\llbracket \cdot, \cdot \rrbracket: \Gamma(Q) \times \Gamma(Q) \rightarrow \Gamma(Q)$, a linear connection $\nabla: \Gamma(Q) \times \Gamma(B) \rightarrow \Gamma(B)$ and a vector valued 3-form $\omega \in$ $\Omega^{3}\left(Q, B^{*}\right)$, such that [12]

(i) $\nabla_{\partial_{B}^{*} \beta_{1}}^{*} \beta_{2}+\nabla_{\partial_{B}^{*} \beta_{2}}^{*} \beta_{1}=0$ for all $\beta_{1}, \beta_{2} \in \Gamma\left(B^{*}\right)$,

(ii) $\llbracket q, \partial_{B}^{*} \beta \rrbracket=\partial_{B}^{*}\left(\nabla_{q}^{*} \beta\right)$ for $q \in \Gamma(Q)$ and $\beta \in \Gamma\left(B^{*}\right)$,

(iii) $\mathrm{Jac}_{\llbracket \cdot, \rrbracket}=\partial_{B}^{*} \circ \omega \in \Omega^{3}(Q, Q)$,

(iv) $R_{\nabla}\left(q_{1}, q_{2}\right) b=\partial_{B}\left\langle\mathbf{i}_{q_{2}} \mathbf{i}_{q_{1}} \omega, b\right\rangle$ for $q \in \Gamma(Q)$ and $\beta \in \Gamma\left(B^{*}\right)$, and

(v) $\mathbf{d}_{\nabla * \omega}=0$.

The equation

$$
\rho_{Q} \circ \partial_{B}^{*}=0
$$

follows easily from (1) and (ii), and (ii) is equivalent to

$$
\partial_{B}\left(\Delta_{q} \tau\right)=\nabla_{q}\left(\partial_{B} \tau\right)
$$

for all $q \in \Gamma(Q)$ and $\tau \in \Gamma\left(Q^{*}\right)$. Further, (4) and (iii) yield together

$$
\left\langle\mathbf{i}_{q_{2}} \mathbf{i}_{q_{2}} \omega, \partial_{B} \tau\right\rangle=R_{\Delta}\left(q_{1}, q_{2}\right) \tau
$$

for $q_{1}, q_{2} \in \Gamma(Q)$ and $\tau \in \Gamma\left(Q^{*}\right)$. The linear Courant algebroid structure on $Q \times_{M}$ $B \times{ }_{M} Q^{*} \rightarrow B$ is given by the anchor $\Theta: \mathbb{E} \rightarrow T B$ defined by

$$
\Theta(q, 0)=\widehat{\nabla_{q}} \in \mathfrak{X}^{l}(B), \quad \Theta\left(\tau^{\dagger}\right)=\left(\partial_{B} \tau\right)^{\uparrow} \in \mathfrak{X}^{c}(B),
$$


and the bracket defined by $\llbracket \tau_{1}^{\dagger}, \tau_{2}^{\dagger} \rrbracket=0$, $\llbracket(q, 0), \tau^{\dagger} \rrbracket=\Delta_{q} \tau^{\dagger}$ where $\Delta$ is the Dorfman connection that is dual to the dull bracket, and $\llbracket\left(q_{1}, 0\right),\left(q_{2}, 0\right) \rrbracket=$ $\left(\llbracket q_{1}, q_{2} \rrbracket,-\mathbf{i}_{q_{2}} \mathbf{i}_{q_{1}} \omega\right)$. for all $q, q_{1}, q_{2} \in \Gamma(Q)$ and all $\tau, \tau_{1}, \tau_{2} \in \Gamma\left(Q^{*}\right)$.

Example 2.5 Jotz Lean [12] We consider here a Courant algebroid $(\mathrm{E} \rightarrow$ $M, \rho, \llbracket \cdot, \cdot \rrbracket,\langle\cdot, \cdot\rangle)$. We use the pairing to identify $\mathrm{E}$ with $\mathrm{E}^{*}$. After the choice of a metric connection on $\mathrm{E}$ and so of a Lagrangian decomposition $I_{\nabla}: T \mathrm{E} \rightarrow$ $\mathrm{E} \times{ }_{M} T M \times_{M} \mathrm{E}$ (see Example 2.2), the VB-Courant algebroid structure on $(T \mathrm{E} \rightarrow$ $T M, \mathrm{E} \rightarrow M)$ is described by $\partial_{T M}=\rho: \mathrm{E} \rightarrow T M$, the Dorfman connection $\Delta^{\text {bas }}: \Gamma(\mathrm{E}) \times \Gamma(\mathrm{E}) \rightarrow \Gamma(\mathrm{E})$,

$$
\Delta_{e}^{\mathrm{bas}} e^{\prime}=\llbracket e, e^{\prime} \rrbracket+\nabla_{\rho\left(e^{\prime}\right)} e,
$$

which we call the basic Dorfman connection associated to $\nabla$. The dual dull bracket is given by

$$
\llbracket e, e^{\prime} \rrbracket_{\Delta}^{\text {bas }}=\llbracket e, e^{\prime} \rrbracket-\rho^{*}\left\langle\nabla . e, e^{\prime}\right\rangle
$$

for all $e, e^{\prime} \in \Gamma(\mathrm{E})$. The linear connection is $\nabla^{\text {bas }}: \Gamma(\mathrm{E}) \times \mathfrak{X}(M) \rightarrow \mathfrak{X}(M)$,

$$
\nabla_{e}^{\text {bas }} X=[\rho(e), X]+\rho\left(\nabla_{X} e\right) .
$$

The basic curvature $\omega_{\Delta}^{\text {bas }} \in \Omega^{3}\left(\mathrm{E}, T^{*} M\right)$ is defined by

$$
\begin{aligned}
\omega_{\Delta}^{\mathrm{bas}}\left(e_{1}, e_{2}, \cdot\right) X= & -\nabla_{X} \llbracket e_{1}, e_{2} \rrbracket+\llbracket \nabla_{X} e_{1}, e_{2} \rrbracket+\llbracket e_{1}, \nabla_{X} e_{2} \rrbracket \\
& +\nabla_{\nabla_{e_{2}}}{ }^{\text {bas }} e_{1}-\nabla_{\nabla_{e_{1}}} e_{2}-\beta^{-1}\left\langle\nabla_{\nabla^{\text {bas }} X} e_{1}, e_{2}\right\rangle \in \Gamma(\mathrm{E})
\end{aligned}
$$

for all $e_{1}, e_{2} \in \Gamma(\mathrm{E})$ and $X \in \mathfrak{X}(M)$.

A homological vector field $\mathcal{Q}$ on a positively graded manifold $\mathcal{M}$ is a derivation of degree 1 of $C^{\infty}(\mathcal{M})$ such that $\mathcal{Q}^{2}=\frac{1}{2}[\mathcal{Q}, \mathcal{Q}]$ vanishes [24]. If the graded manifold is a [2]-manifold, then the pair $(\mathcal{M}, \mathcal{Q})$ is a Lie 2-algebroid [28].

Consider the split [2]-manifold $Q[-1] \oplus B^{*}[-2]$ corresponding to the underlying decomposed metric double vector bundle $Q \times_{M} B \times_{M} Q^{*}$. The linear Courant algebroid structure defines as follows a homological vector field $\mathcal{Q}$ on $C^{\infty}(Q[-1 \mid \oplus$ $\left.B^{*}[-2]\right)($ see [12]):

for $f \in C^{\infty}(M)$,

$$
\mathcal{Q}(f)=\rho_{Q}^{*} \mathbf{d} f \in \Gamma\left(Q^{*}\right)
$$

for $\tau \in \Gamma\left(Q^{*}\right)$ and

$$
\mathcal{Q}(\tau)=\mathbf{d}_{Q} \tau+\partial_{B} \tau \in \Omega^{2}(Q) \oplus \Gamma(B)
$$

$$
\mathcal{Q}(b)=\mathbf{d}_{\nabla} b-\langle\omega, b\rangle \in \Omega^{1}(Q, B) \oplus \Omega^{3}(Q) .
$$

for $b \in \Gamma(B)$. The tuple $\left(\partial_{B}^{*}: B^{*} \rightarrow Q, \rho_{Q}, \llbracket \cdot, \cdot \rrbracket, \nabla, \omega\right)$, is a split Lie 2-algebroid $[12,28]$. Note that conversely any homological vector field on $\mathcal{M}=Q[-1] \oplus$ $B^{*}[-2]$ defines as in (16), (17) and (18) a split Lie 2-algebroid

The category of Lie 2-algebroid is equivalent via the correspondence described above to the category of VB-Courant algebroids [12, 14]. Note that a morphism $\mu:\left(\mathcal{M}_{1}, \mathcal{Q}_{1}\right) \rightarrow\left(\mathcal{M}_{2}, \mathcal{Q}_{2}\right)$ of Lie 2-algebroids is a morphism $\mu: \mathcal{M}_{1} \rightarrow \mathcal{M}_{2}$ of the underlying [2]-manifolds, such that

$$
\mu^{\star} \circ \mathcal{Q}_{2}=\mathcal{Q}_{1} \circ \mu^{\star}: C^{\infty}\left(\mathcal{M}_{2}\right) \rightarrow C^{\infty}\left(\mathcal{M}_{1}\right) .
$$


We refer to Section 3.5 of [12] for the characterisation of a morphism of split Lie 2-algebroids in terms of its components $\left(\partial_{B}^{*}: B^{*} \rightarrow Q, \rho_{Q}, \llbracket \cdot, \cdot \rrbracket, \nabla, \omega\right)$.

\section{LA-Courant Algebroids vs Poisson Lie 2-Algebroids}

In this section, we prove that a split Poisson Lie 2-algebroid is equivalent to the matched pair of a split Lie 2-algebroid with a self-dual 2-representation.

Take a double vector bundle $(\mathbb{E}, B, Q, M)$ with core $Q^{*}$, with a VB-Lie algebroid structure on $(\mathbb{E} \rightarrow Q, B \rightarrow M)$ and a VB-Courant algebroid structure on $(\mathbb{E} \rightarrow B, Q \rightarrow M)$. In this section we show that the double vector bundle is an LA-Courant algebroid [14] if and only if the VB-algebroid is metric and the self-dual 2-representation defined by any Lagrangian decomposition of $\mathbb{E}$ and the VB-algebroid side forms a matched pair with the split Lie 2-algebroid describing the Courant algebroid side.

We begin with the following definition.

Definition 3.1 Let $\left(B \rightarrow M, \rho_{B},[\cdot, \cdot]\right)$ be a Lie algebroid and let $\left(Q \rightarrow M, \rho_{Q}\right)$ be an anchored vector bundle. Assume that $B$ acts on $\partial_{Q}: Q^{*} \rightarrow Q$ up to homotopy via a self-dual 2-representation $\left(\nabla, \nabla^{*}, R\right)$, and let $\left(\partial_{B}: Q^{*} \rightarrow B, \llbracket \cdot, \cdot \rrbracket, \nabla, \omega\right)^{4}$ be a split Lie 2 -algebroid. Then we say that the 2-representation and the split Lie 2-algebroid form a matched pair if

(M1) $\partial_{Q}\left(\Delta_{q} \tau\right)=\nabla_{\partial_{B} \tau} q+\llbracket q, \partial_{Q} \tau \rrbracket+\partial_{B}^{*}\langle\tau, \nabla . q\rangle$,

(M2) $\partial_{B}\left(\nabla_{b}^{*} \tau\right)=\left[b, \partial_{B} \tau\right]+\nabla_{\partial_{Q} \tau} b$,

(M3) $\partial_{B} R\left(b_{1}, b_{2}\right) q=-\nabla_{q}\left[b_{1}, b_{2}\right]+\left[\nabla_{q} b_{1}, b_{2}\right]+\left[b_{1}, \nabla_{q} b_{2}\right]+\nabla_{\nabla_{b_{2}}} b_{1}-\nabla_{\nabla_{b_{1}} q} b_{2}$,

(M4) $\partial_{Q}\left\langle\mathbf{i}_{q_{2}} \mathbf{i}_{q_{1}} \omega, b\right\rangle=-\nabla_{b} \llbracket q_{1}, q_{2} \rrbracket+\llbracket q_{1}, \nabla_{b} q_{2} \rrbracket+\llbracket \nabla_{b} q_{1}, q_{2} \rrbracket+\nabla_{\nabla_{q_{2}}} q_{1}-$ $\nabla_{\nabla_{q_{1}} b} q_{2}+\partial_{B}^{*}\left\langle R(\cdot, b) q_{1}, q_{2}\right\rangle$.

(M5) $\mathbf{d}_{\nabla Q} \omega=\mathbf{d}_{\nabla B} R \in \Omega^{2}\left(B, \wedge^{3} Q^{*}\right) \simeq \Omega^{3}\left(Q, \wedge^{2} B^{*}\right)$, where $\omega$ is seen as an element of $\Omega^{1}\left(B, \wedge^{3} Q^{*}\right)$ and $R$ is understood as an element of $\Omega^{2}\left(Q, \wedge^{2} B^{*}\right)$.

Remark 3.2 (1) (M5) is

$$
\begin{aligned}
& \nabla_{b_{2}}^{*}\left\langle\mathbf{i}_{q_{2}} \mathbf{i}_{q_{1}} \omega, b_{1}\right\rangle-\nabla_{b_{1}}^{*}\left\langle\mathbf{i}_{q_{2}} \mathbf{i}_{q_{1}} \omega, b_{2}\right\rangle+\left\langle\mathbf{i}_{q_{2}} \mathbf{i}_{q_{1}} \omega,\left[b_{1}, b_{2}\right]\right\rangle \\
& +\left\langle\mathbf{i}_{q_{2}} \mathbf{i}_{\nabla_{b_{1}} q_{1}} \omega, b_{2}\right\rangle+\left\langle\mathbf{i}_{\nabla_{b_{1}} q_{2}} \mathbf{i}_{q_{1}} \omega, b_{2}\right\rangle-\left\langle\mathbf{i}_{q_{2}} \mathbf{i}_{\nabla_{b_{2}} q_{1}} \omega, b_{1}\right\rangle-\left\langle\mathbf{i}_{\nabla_{b_{2}} q_{2}} \mathbf{i}_{q_{1}} \omega, b_{1}\right\rangle \\
& +\Delta_{q_{1}} R\left(b_{1}, b_{2}\right) q_{2}-\Delta_{q_{2}} R\left(b_{1}, b_{2}\right) q_{1}-R\left(b_{1}, b_{2}\right) \llbracket q_{1}, q_{2} \rrbracket \\
& -R\left(\nabla_{q_{1}} b_{1}, b_{2}\right) q_{2}-R\left(b_{1}, \nabla_{q_{1}} b_{2}\right) q_{2}+R\left(\nabla_{q_{2}} b_{1}, b_{2}\right) q_{1}+R\left(b_{1}, \nabla_{q_{2}} b_{2}\right) q_{1} \\
= & \left\langle\left(R\left(b_{1}, \nabla . b_{2}\right)+R\left(\nabla . b_{1}, b_{2}\right)\right) q_{1}, q_{2}\right\rangle-\rho_{Q}^{*} \mathbf{d}\left\langle R\left(b_{1}, b_{2}\right) q_{1}, q_{2}\right\rangle
\end{aligned}
$$

for all $q_{1}, q_{2} \in \Gamma(Q)$ and $b_{1}, b_{2} \in \Gamma(B)$.

(2) The equality $\rho_{Q} \circ \partial_{Q}=\rho_{B} \circ \partial_{B}$ follows from (M1) if $Q$ has positive rank, and from (M2) if $B$ has positive rank. If both $Q$ and $B$ have rank zero, then $\rho_{Q} \circ \partial_{Q}=\rho_{B} \circ \partial_{B}$ is trivially satisfied.

\footnotetext{
${ }^{4}$ For the sake of simplicity, we write in this definition $\nabla$ for two different connections, unless it is not clear from the indexes which connection is meant.
} 
(3) The equation

$$
\left[\rho_{Q}(q), \rho_{B}(b)\right]=\rho_{B}\left(\nabla_{q} b\right)-\rho_{Q}\left(\nabla_{b} q\right)
$$

follows from (M3) if $B$ has positive rank, and from (M4) if $Q$ has positive rank. If both $Q$ and $B$ have rank zero, then it is trivially satisfied.

(4) If $\rho_{Q} \circ \partial_{Q}=\rho_{B} \circ \partial_{B}$, then (M1) is equivalent to

$$
\left(\Delta_{\partial_{Q} \tau_{1}} \tau_{2}-\nabla_{\partial_{B} \tau_{2}}^{*} \tau_{1}\right)+\left(\Delta_{\partial_{Q} \tau_{2}} \tau_{1}-\nabla_{\partial_{B} \tau_{1}}^{*} \tau_{2}\right)=\rho_{Q}^{*} \mathbf{d}\left\langle\tau_{1}, \partial_{Q} \tau_{2}\right\rangle
$$

for all $\tau_{1}, \tau_{2} \in \Gamma\left(Q^{*}\right)$.

(5) Assuming $\left[\rho_{Q}(q), \rho_{B}(b)\right]=\rho_{B}\left(\nabla_{q} b\right)-\rho_{Q}\left(\nabla_{b} q\right)$ for all $b \in \Gamma(B)$ and $q \in$ $\Gamma(Q),(\mathrm{M} 4)$ is equivalent to

$$
\left\langle\mathbf{i}_{\partial_{Q} \tau} \mathbf{i}_{q} \omega, b\right\rangle-R\left(b, \partial_{B} \tau\right) q=\Delta_{q} \nabla_{b}^{*} \tau-\nabla_{b}^{*} \Delta_{q} \tau+\Delta_{\nabla_{b} q} \tau-\nabla_{\nabla_{q} b}^{*} \tau-\left\langle\nabla_{\nabla . b} q, \tau\right\rangle
$$

for all $b \in \Gamma(B), q \in \Gamma(Q)$ and $\tau \in \Gamma\left(Q^{*}\right)$.

\subsection{Poisson Lie 2-Algebroids via Matched Pairs}

We begin this subsection with the definition of a Poisson Lie 2-algebroid.

Definition 3.3 Let $\mathcal{M}$ be a Poisson [2]-manifold with algebra of functions $C^{\infty}(\mathcal{M})$ and degree -2 Poisson bracket $\{\cdot, \cdot\}$. Assume that $\mathcal{M}$ has in addition a Lie 2algebroid structure, i.e. that it is endowed with a homological vector field $\mathcal{Q}$. Then $(\mathcal{M}, \mathcal{Q},\{\cdot, \cdot\})$ is a Poisson Lie 2-algebroid if the homological vector field preserves the Poisson structure, i.e. if

$$
\mathcal{Q}\left\{\xi_{1}, \xi_{2}\right\}=\left\{\mathcal{Q}\left(\xi_{1}\right), \xi_{2}\right\}+(-1)^{\operatorname{deg} \xi_{1}}\left\{\xi_{1}, \mathcal{Q}\left(\xi_{2}\right)\right\}
$$

for all $\xi_{1}, \xi_{2} \in \mathcal{A}$.

A morphism of Poisson Lie 2-algebroids is a morphism of the underlying [2]manifold that is a morphism of Poisson [2]-manifolds and a morphism of Lie 2algebroids.

The main theorem of this section shows that matched pairs as in Definition 3.1 are equivalent to split Poisson Lie 2-algebroids.

Theorem 3.4 Let $\mathcal{M}=Q[-1] \oplus B^{*}[-2]$ be a split [2]-manifold endowed with a homological vector field $\mathcal{Q}$ and a Poisson bracket $\{\cdot, \cdot\}$ of degree -2 . Let $\left(\partial_{B}: Q^{*} \rightarrow\right.$ $B, \llbracket \cdot, \cdot \rrbracket, \nabla, \omega)$ be the components of $\mathcal{Q}$, and let $\left(\partial_{Q}: Q^{*} \rightarrow Q, \nabla^{*}, \nabla, R\right)$ be the self-dual 2-representation of $B$ that is equivalent to the Poisson bracket.

Then $(\mathcal{M}, \mathcal{Q},\{\cdot, \cdot\})$ is a Poisson Lie 2-algebroid if and only if the self dual 2representation and the split Lie 2-algebroid form a matched pair. 
Proof The idea of this proof is very simple, but requires rather long computations. We will leave some of the detailed verifications to the reader. We check (23) in coordinates, by using the formulae found in (16), (17), (18) and (10) for $\mathcal{Q}$ and $\{\cdot, \cdot\}$, respectively.

First we have $\mathcal{Q}(f)=\rho_{Q}^{*} \mathbf{d} f \in \Gamma\left(Q^{*}\right)$ and $\{f, g\}=0$ for $f, g \in C^{\infty}(M)$. This yields $\{\mathcal{Q}(f), g\}+\{f, \mathcal{Q}(g)\}=\left\{\rho_{Q}^{*} \mathbf{d} f, g\right\}+\left\{f, \rho_{Q}^{*} \mathbf{d} g\right\}=0=\mathcal{Q}\{f, g\}$ by the graded skew-symmetry and $\{\tau, f\}=0$ for $\tau \in \Gamma\left(Q^{*}\right)$. Then we have for $\tau \in \Gamma\left(Q^{*}\right)$ :

$$
\begin{aligned}
\{\mathcal{Q}(\tau), f\}-\{\tau, \mathcal{Q}(f)\} & =\left\{\mathbf{d}_{Q} \tau+\partial_{B} \tau, f\right\}-\left\{\tau, \rho_{Q}^{*} \mathbf{d} f\right\} \\
& =0+\rho_{B}\left(\partial_{B} \tau\right)(f)-\rho_{Q}\left(\partial_{Q} \tau\right)(f) .
\end{aligned}
$$

But we also have $\mathcal{Q}\{\tau, f\}=\mathcal{Q}(0)=0$. Hence, $\{\mathcal{Q}(\tau), f\}-\{\tau, \mathcal{Q}(f)\}=\mathcal{Q}\{\tau, f\}$ is equivalent to $\rho_{B}\left(\partial_{B} \tau\right)(f)=\rho_{Q}\left(\partial_{Q} \tau\right)(f)$.

In a similar manner, we have $\mathcal{Q}\{b, f\}=\rho_{Q}^{*} \mathbf{d}\left(\rho_{B}(b)(f)\right)$ for $b \in \Gamma(B)$ and $\{\mathcal{Q}(b), f\}+\{b, \mathcal{Q}(f)\}$ is

$$
\begin{aligned}
& \left\{\mathbf{d}_{\nabla} b-\langle\omega, b\rangle, f\right\}+\left\{b, \rho_{Q}^{*} \mathbf{d} f\right\}=\left\{\mathbf{d}_{\nabla} b, f\right\}+\nabla_{b}^{*}\left(\rho_{Q}^{*} \mathbf{d} f\right) \\
= & \{\nabla . b, f\}+\nabla_{b}^{*}\left(\rho_{Q}^{*} \mathbf{d} f\right)=\rho_{B}(\nabla . b)(f)+\nabla_{b}^{*}\left(\rho_{Q}^{*} \mathbf{d} f\right) \in \Omega^{1}(Q) .
\end{aligned}
$$

Hence, $\mathcal{Q}\{b, f\}=\{\mathcal{Q}(b), f\}+\{b, \mathcal{Q}(f)\}$ if and only if

$$
\rho_{Q}(q) \rho_{B}(b)(f)=\rho_{B}\left(\nabla_{q} b\right)(f)+\rho_{B}(b) \rho_{Q}(q)(f)-\rho_{Q}\left(\nabla_{b} q\right)(f)
$$

for all $q \in \Gamma(Q)$. Since $f$ was arbitrary, this is $(20)$ : $\left[\rho_{Q}(q), \rho_{B}(b)\right]=\rho_{B}\left(\nabla_{q} b\right)-$ $\rho_{Q}\left(\nabla_{b} q\right)$. Then we have $\mathcal{Q}\{b, \tau\}=\mathcal{Q}\left(\nabla_{b}^{*} \tau\right)=\partial_{B}\left(\nabla_{b}^{*} \tau\right)+\mathbf{d}_{Q}\left(\nabla_{b}^{*} \tau\right) \in \Gamma(B) \oplus$ $\Omega^{2}(Q)$. The Poisson bracket $\{\mathcal{Q}(b), \tau\}$ is $\left\{\mathbf{d}_{\nabla} b-\langle\omega, b\rangle, \tau\right\}$. A simple computation shows that $\{\eta, \tau\}=(-1)^{k+1} \mathbf{i}_{\partial_{Q} \tau} \eta$ for $\eta \in \Omega^{k}(Q)$. Hence, $\{\langle\omega, b\rangle, \tau\}=$ $(-1)^{4} \mathbf{i}_{\partial_{Q} \tau}\langle\omega, b\rangle=\mathbf{i}_{\partial_{Q} \tau}\langle\omega, b\rangle$. The bracket $\left\{\mathbf{d}_{\nabla} b, \tau\right\}$ equals $\nabla_{\partial_{Q} \tau} b+\psi_{b, \tau}$, with $\psi_{b, \tau} \in \Omega^{2}(Q)$ the form defined by

$$
\psi_{b, \tau}\left(q_{1}, q_{2}\right)=\left\langle\nabla_{\nabla_{q_{1}}}^{*} \tau, q_{2}\right\rangle-\left\langle\nabla_{\nabla_{q_{2}}}^{*} \tau, q_{1}\right\rangle
$$

for $q_{1}, q_{2} \in \Gamma(Q)$. The Poisson bracket $\{b, \mathcal{Q}(\tau)\}=\left\{b, \mathbf{d}_{Q} \tau+\partial_{B} \tau\right\}=\left\{b, \mathbf{d}_{Q} \tau\right\}+$ $\left[b, \partial_{B} \tau\right]-R\left(b, \partial_{B} \tau\right)$ simplifies to $\nabla_{b}^{*}\left(\mathbf{d}_{Q} \tau\right)+\left[b, \partial_{B} \tau\right]-R\left(\partial_{B} \tau, b\right)$ because $\{b, \eta\}=$ $\nabla_{b} \eta \in \Omega^{k}(Q)$ for all $\eta \in \Omega^{k}(Q)$. By comparing the $\Gamma(B)$ and the $\Omega^{2}(Q)$-terms, we find that

$$
\mathcal{Q}\{b, \tau\}=\{\mathcal{Q}(b), \tau\}+\{b, \mathcal{Q}(\tau)\}
$$

if and only if $\partial_{B} \nabla_{b}^{*} \tau=\nabla_{\partial_{Q} \tau} b+\left[b, \partial_{B} \tau\right]$, which is (M2) and

$$
\mathbf{d}_{Q}\left(\nabla_{b}^{*} \tau\right)=-\mathbf{i}_{\partial_{Q} \tau}\langle\omega, b\rangle+\psi_{b, \tau}+\nabla_{b}^{*}\left(\mathbf{d}_{Q} \tau\right)+R\left(\partial_{B} \tau, b\right) .
$$

On $q_{1}, q_{2} \in \Gamma(Q)$, the 2 -form $\mathbf{d}_{Q}\left(\nabla_{b}^{*} \tau\right)+\mathbf{i}_{\partial_{Q} \tau}\langle\omega, b\rangle-\psi_{b, \tau}-\nabla_{b}^{*}\left(\mathbf{d}_{Q} \tau\right)-R\left(\partial_{B} \tau, b\right)$ is

$$
\begin{aligned}
& \left(\left[\rho_{Q}\left(q_{1}\right), \rho_{B}(b)\right]-\rho_{B}\left(\nabla_{q_{1}} b\right)+\rho_{Q}\left(\nabla_{b} q_{1}\right)\right)\left\langle\tau, q_{2}\right\rangle \\
& +\left(\left[\rho_{B}(b), \rho_{Q}\left(q_{2}\right)\right]+\rho_{B}\left(\nabla_{q_{2}} b\right)-\rho_{Q}\left(\nabla_{b} q_{2}\right)\right)\left\langle\tau, q_{1}\right\rangle \\
& +\left\langle\tau, \partial_{Q}^{*}\left\langle\mathbf{i}_{q_{2}} \mathbf{i}_{q_{1}} \omega, b\right\rangle+\nabla_{b} \llbracket q_{1}, q_{2} \rrbracket+\nabla_{\nabla_{q_{1}} b} q_{2}-\nabla_{\nabla_{q_{2}} b q_{1}}\right\rangle \\
& -\left\langle\tau, \llbracket \nabla_{b} q_{1}, q_{2} \rrbracket+\llbracket q_{1}, \nabla_{b} q_{2} \rrbracket+\partial_{B}^{*}\left\langle R(\cdot, b) q_{1}, q_{2}\right\rangle\right\rangle .
\end{aligned}
$$


Hence if (20) holds, then (24) is (M4). Next we study the equation $\mathcal{Q}\left\{\tau_{1}, \tau_{2}\right\}=$ $\left\{\mathcal{Q}\left(\tau_{1}\right), \tau_{2}\right\}-\left\{\tau_{1}, \mathcal{Q}\left(\tau_{2}\right)\right\}$ for $\tau_{1}, \tau_{2} \in \Gamma\left(Q^{*}\right)$. The left hand side is $\rho_{Q}^{*} \mathbf{d}\left\langle\tau_{1}, \partial_{Q} \tau_{2}\right\rangle$. The right-hand side is $\left\{\mathcal{Q}\left(\tau_{1}\right), \tau_{2}\right\}+\left\{\mathcal{Q}\left(\tau_{2}\right), \tau_{1}\right\}$. The equality

$$
\begin{aligned}
\left\{\mathcal{Q}\left(\tau_{1}\right), \tau_{2}\right\} & =\left\{\mathbf{d}_{Q} \tau_{1}+\partial_{B} \tau_{1}, \tau_{2}\right\}=-\mathbf{i}_{\partial_{Q} \tau_{2}} \mathbf{d}_{Q} \tau_{1}+\nabla_{\partial_{B} \tau_{1}}^{*} \tau_{2} \\
& =\rho_{Q}^{*} \mathbf{d}\left\langle\tau_{1}, \partial_{Q} \tau_{2}\right\rangle-\Delta_{\partial Q} \tau_{2} \tau_{1}+\nabla_{\partial_{B} \tau_{1}}^{*} \tau_{2}
\end{aligned}
$$

shows hence that $\mathcal{Q}\left\{\tau_{1}, \tau_{2}\right\}=\left\{\mathcal{Q}\left(\tau_{1}\right), \tau_{2}\right\}+\left\{\mathcal{Q}\left(\tau_{2}\right), \tau_{1}\right\}$ if and only if (21). Recall from Remark 3.2 that together with $\rho_{B} \circ \partial_{B}=\rho_{Q} \circ \partial_{Q}$, this is equivalent to (M1).

Finally, we choose $b_{1}, b_{2} \in \Gamma(B)$ and we study the equation $\mathcal{Q}\left\{b_{1}, b_{2}\right\}=$ $\left\{\mathcal{Q}\left(b_{1}\right), b_{2}\right\}+\left\{b_{1}, \mathcal{Q}\left(b_{2}\right)\right\}=\left\{\mathcal{Q}\left(b_{1}\right), b_{2}\right\}-\left\{\mathcal{Q}\left(b_{2}\right), b_{1}\right\}$. The left-hand side is

$$
\mathcal{Q}\left(\left[b_{1}, b_{2}\right]-R\left(b_{1}, b_{2}\right)\right)=\underbrace{\mathbf{d}_{\nabla}\left[b_{1}, b_{2}\right]}_{\in \Omega^{1}(Q, B)}-\underbrace{\left\langle\omega,\left[b_{1}, b_{2}\right]\right\rangle-\mathbf{d}_{Q}\left(R\left(b_{1}, b_{2}\right)\right)}_{\in \Omega^{3}(Q)}+\underbrace{\partial_{B} R\left(b_{1}, b_{2}\right)}_{\in \Omega^{1}(Q, B)} .
$$

Note that in the expression $\mathbf{d}_{Q}\left(R\left(b_{1}, b_{2}\right)\right)$, the object $R\left(b_{1}, b_{2}\right)$ is understood as an element of $\Omega^{2}(Q)$, and in the expression $\partial_{B} R\left(b_{1}, b_{2}\right)$, it is understood as a morphism $Q \rightarrow Q^{*}$. The Poisson bracket $\left\{\mathcal{Q}\left(b_{1}\right), b_{2}\right\}$ is

$$
\left\{\mathbf{d}_{\nabla} b_{1}-\left\langle\omega, b_{1}\right\rangle, b_{2}\right\}=\underbrace{\nabla_{\nabla_{b_{2}}} \cdot b_{1}+\left[\nabla \cdot b_{1}, b_{2}\right]}_{\in \Omega^{1}(Q, B)}-\underbrace{R\left(\nabla \cdot b_{1}, b_{2}\right)+\nabla_{b_{2}}\left\langle\omega, b_{1}\right\rangle}_{\in \Omega^{3}(Q)}
$$

The projection to $\Omega^{1}(Q, B)$ of $\mathcal{Q}\left\{b_{1}, b_{2}\right\}=\left\{\mathcal{Q}\left(b_{1}\right), b_{2}\right\}-\left\{\mathcal{Q}\left(b_{2}\right), b_{1}\right\}$ is

$$
\mathbf{d}_{\nabla}\left[b_{1}, b_{2}\right]+\partial_{B} R\left(b_{1}, b_{2}\right)=\nabla_{\nabla_{b_{2}}} \cdot b_{1}+\left[\nabla \cdot b_{1}, b_{2}\right]-\nabla_{\nabla_{b_{1}}} \cdot b_{2}-\left[\nabla \cdot b_{2}, b_{1}\right],
$$

which is (M3). The projection to $\Omega^{3}(Q)$ of $\mathcal{Q}\left\{b_{1}, b_{2}\right\}=\left\{\mathcal{Q}\left(b_{1}\right), b_{2}\right\}-\left\{\mathcal{Q}\left(b_{2}\right), b_{1}\right\}$ is

$$
\begin{aligned}
-\left\langle\omega,\left[b_{1}, b_{2}\right]\right\rangle-\mathbf{d}_{Q}\left(R\left(b_{1}, b_{2}\right)\right)= & -R\left(\nabla . b_{1}, b_{2}\right)+\nabla_{b_{2}}\left\langle\omega, b_{1}\right\rangle \\
& +R\left(\nabla . b_{2}, b_{1}\right)-\nabla_{b_{1}}\left\langle\omega, b_{2}\right\rangle,
\end{aligned}
$$

that is,

$$
\left(\mathbf{d}_{\nabla} \omega\right)\left(b_{1}, b_{2}\right)=\mathbf{d}_{Q}\left(R\left(b_{1}, b_{2}\right)\right)-R\left(\nabla . b_{1}, b_{2}\right)+R\left(\nabla . b_{2}, b_{1}\right) .
$$

The right-hand side of this equation is easily calculated to be the pairing of $\left(\mathbf{d}_{\nabla^{B}} R\right) \in$ $\Omega^{3}\left(Q, \wedge^{2} B^{*}\right)$ with $\left(b_{1}, b_{2}\right)$. Hence, the projection to $\Omega^{3}(Q)$ of $\mathcal{Q}\left\{b_{1}, b_{2}\right\}=$ $\left\{\mathcal{Q}\left(b_{1}\right), b_{2}\right\}-\left\{\mathcal{Q}\left(b_{2}\right), b_{1}\right\}$ is (M5).

\subsection{LA-Courant Algebroids and Equivalence of Categories}

Li-Bland's definition of an LA-Courant algebroid [14] requires the consideration of triple vector bundles [18]. This is explained in this section.

\subsubsection{The LA-Courant Algebroid Condition}

A Dirac structure with support in a Courant algebroid $\mathrm{E} \rightarrow M$ is a subbundle $D \rightarrow S$ over a sub-manifold $S$ of $M$, such that $D(s)$ is maximal isotropic in $\mathrm{E}(s)$ for all $s \in S$ and

$$
\left.e_{1}\right|_{S} \in \Gamma_{S}(D),\left.e_{2}\right|_{S} \in \Gamma_{S}(D) \quad \Rightarrow \quad \llbracket e_{1},\left.e_{2} \rrbracket\right|_{S} \in \Gamma_{S}(D)
$$


for all $e_{1}, e_{2} \in \Gamma(\mathrm{E})$.

Later we will need the following lemma in Section 5. We leave the proof to the reader.

Lemma 3.5 Let $\mathrm{E} \rightarrow M$ be a Courant algebroid and $D \rightarrow S$ a subbundle with $S$ a sub-manifold of $M$. Assume that $D \rightarrow S$ is spanned by the restrictions to $S$ of a family $\mathcal{S} \subseteq \Gamma(\mathrm{E})$ of sections of $\mathrm{E}$. Then $D$ is a Dirac structure with support $S$ if and only if

(1) $\rho_{\mathrm{E}}(e)(s) \in T_{S} S$ for all $e \in \mathcal{S}$ and $s \in S$,

(2) $D_{s}$ is Lagrangian in $\mathbb{E}_{s}$ for all $s \in S$ and

(3) $\llbracket e_{1},\left.e_{2} \rrbracket\right|_{S} \in \Gamma_{S}(D)$ for all $e_{1}, e_{2} \in \mathcal{S}$.

Consider a Lie algebroid $\left(q_{A}: A \rightarrow M, \rho_{A},[\cdot, \cdot]\right)$. In [14] Li-Bland defines a relation $\Pi_{A} \subseteq T A \times T A$ by

$$
\begin{aligned}
T_{m} b \rho_{A}(a)(m)+\left.{ }_{A} \frac{d}{d t}\right|_{t=0} b(m)+t([b, a]+c)(m) & \\
& \sim_{\Pi_{A}} T_{m} a \rho_{A}(b)(m)+\left.{ }_{A} \frac{d}{d t}\right|_{t=0} a(m)+t c(m)
\end{aligned}
$$

for all $a, b, c \in \Gamma(A)$. (Note that in [14], the relation is defined in a different manner. Checking that this alternative definition is correct is rather long. Details can be obtained in the appendix of [8]).

Now consider a double vector bundle $(\mathbb{E}, B, Q, M)$ endowed with a VB-Lie algebroid structure $(\mathbf{b},[\cdot, \cdot])$ on $\mathbb{E} \rightarrow Q$ and a linear metric $\langle\cdot, \cdot\rangle$ on $\mathbb{E} \rightarrow B$ (hence, $\mathbb{E}$ has core $Q^{*}$ ). Let $\rho_{B}: B \rightarrow T M$ be the anchor of the induced Lie algebroid structure on $B$.

The relation $\Pi_{\mathbb{E}}$ defined as above by the Lie algebroid structure on $\mathbb{E}$ over $Q$ is then a relation $\Pi_{\mathbb{E}}$ of the triple vector bundles [14]

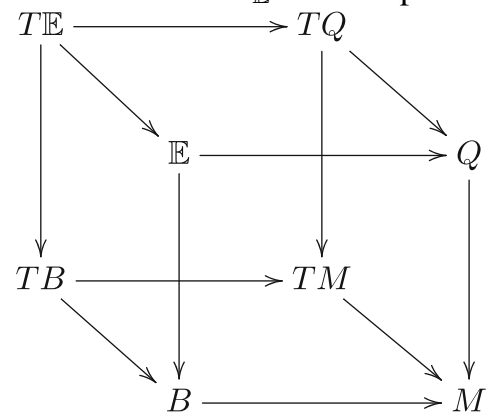

and

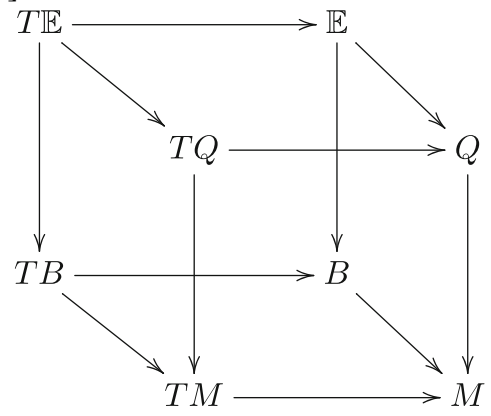

Li-Bland's definition [14] is the following.

Definition 3.6 Let $(\mathbb{E}, Q, B, M)$ be a double vector bundle with a VB-Courant algebroid structure (over $B$ ) and a VB-algebroid structure $(\mathbb{E} \rightarrow Q, B \rightarrow M)$. Then $(\mathbb{E}, B, Q, M)$ is an LA-Courant algebroid if $\Pi_{\mathbb{E}}$ is a Dirac structure with support of the Courant algebroid $\overline{T \mathbb{E}} \times T \mathbb{E}$. 
We have the following theorem.

Theorem 3.7 Let $(\mathbb{E}, Q, B, M)$ be a double vector bundle with a VB-Courant algebroid structure on $(\mathbb{E} \rightarrow B, Q \rightarrow M)$ and a VB-Lie algebroid structure on $(\mathbb{E} \rightarrow Q, B \rightarrow M)$. Then in particular, $\mathbb{E}$ is a metric double vector bundle with the linear metric underlying the linear Courant algebroid structure on $\mathbb{E} \rightarrow B$. Choose a Lagrangian decomposition $\Sigma: B \times_{M} Q \rightarrow \mathbb{E}$ of $\mathbb{E}$. Then $(\mathbb{E}, Q, B, M)$ is an LA-Courant algebroid if and only if

(1) the linear Lie algebroid structure on $\mathbb{E} \rightarrow Q$ is compatible in the sense of Section 2.5.1 with the linear metric, and

(2) the self-dual 2-representation and the split Lie 2-algebroid obtained from the Lagrangian splitting form a matched pair as in Definition 3.1.

The proof of this theorem is very long and technical (see the appendix of [8]), showing that Li-Bland's definition of an LA-Courant algebroid is, although very elegant, rather hard to handle in practice. Hence our result provides a new definition of LA-Courant algebroids, that is easier to use for computations.

Further, we now explain how this theorem shows that LA-Courant algebroids are equivalent to Poisson Lie 2-algebroids. This has already been found by Li-Bland in [14]. First, morphisms of LA-Courant algebroids are morphisms of metric double vector bundles that preserve the Courant algebroid structure and the Lie algebroid structure [14]. Hence, the category of LA-Courant algebroids is a full subcategory of the intersection of the category of metric VB-algebroids and the category of VBCourant algebroids.

On the other hand, Definition 3.3 shows that the category of Poisson Lie 2algebroids is a full subcategory of the intersection of the categories of Poisson [2]-manifolds and of Lie 2-algebroids.

This, Theorem 3.4, and Theorem 3.7 show that the equivalences of the categories of metric VB-algebroids and of Poisson [2]-manifolds and of the categories of VBCourant algebroids and Lie 2-algebroids restrict to an equivalence of the category of LA-Courant algebroids with the category of Poisson Lie 2-algebroids.

\subsection{Examples of LA-Courant Algebroids and Poisson Lie 2-Algebroids}

Next we discuss some classes of Examples of LA-Courant algebroids, and the corresponding Poisson Lie 2-algebroids.

\subsubsection{The Tangent Double of a Courant Algebroid}

Let $\mathrm{E} \rightarrow M$ be a Courant algebroid and choose a metric connection $\nabla: \mathfrak{X}(M) \times$ $\Gamma(\mathrm{E}) \rightarrow \Gamma(\mathrm{E})$. We have seen in Examples 2.2 and 2.4 that the triple $\left(\nabla, \nabla, R_{\nabla}\right)$ is then the self dual $T M$-representation up to homotopy describing ( $T \mathrm{E} \rightarrow \mathrm{E}, T M \rightarrow$ $M)$ after the choice of the Lagrangian decomposition $\Sigma^{\nabla}: \mathrm{E} \times{ }_{M} M \times{ }_{M} \mathrm{E} \rightarrow T \mathrm{E}$. We have also seen in Example 2.5 that the split Lie 2-algebroid encoding the Courant algebroid side $(T \mathrm{E} \rightarrow T M, \mathrm{E} \rightarrow T M)$ is $\left(\rho_{\mathrm{E}}: \mathrm{E} \rightarrow T M, \llbracket \cdot, \cdot \rrbracket_{\Delta^{\text {bas }}}, \nabla^{\text {bas }}, \omega_{\Delta}^{\text {bas }}\right)$. 
A straightforward computation resembling the one in [5, Section 3.2] for the tangent double of a Lie algebroid shows that this 2-representation and this split Lie 2-algebroid are matched, and so that $T E$ is an LA-Courant algebroid (see also [14]).

The Poisson structure on the [2]-manifold corresponding to $T E$ is, via the equivalence of [2]-manifolds with metric double vector bundles, just the Poisson structure that is dual to the Lie algebroid $T \mathrm{E} \rightarrow \mathrm{E}$. Hence, it is symplectic (see [10], in particular Section 4.5.1).

Hence, the class of LA-Courant algebroids that is equivalent to the symplectic Lie 2-algebroids is just the class of tangent prolongations of Courant algebroids.

\subsubsection{The Standard Courant Algebroid over a Lie Algebroid}

Let $A$ be a Lie algebroid. Then $T A \oplus T^{*} A$ is a double vector bundle with sides $A$ and $T M \oplus A^{*}$ and with core $A \oplus T^{*} M$. It has a linear Courant algebroid structure on $T A \oplus_{A} T^{*} A \rightarrow A$ (see [12]) and a metric VB-algebroid structure $\left(T A \oplus_{A} T^{*} A \rightarrow\right.$ $\left.T M \oplus A^{*}, A \rightarrow M\right)$ (see [10]).

Set $\partial_{A}=\operatorname{pr}_{A}: A \oplus T^{*} M \rightarrow A$, consider a skew-symmetric dull bracket $\llbracket \cdot, \rrbracket$ on $\Gamma\left(T M \oplus A^{*}\right)$, with $T M \oplus A^{*}$ anchored by $\operatorname{pr}_{T M}$, and let $\Delta: \Gamma\left(T M \oplus E^{*}\right) \times$ $\Gamma\left(A \oplus T^{*} M\right) \rightarrow \Gamma\left(A \oplus T^{*} M\right)$ be the dual Dorfman connection. This Dorfman connection is equivalent to a Lagrangian splitting of the metric double vector bundle $T A \oplus T^{*} A[9,10]$. It also defines as follows a split Lie 2-algebroid structure on the vector bundles $\left(T M \oplus A^{*}, \mathrm{pr}_{T M}\right)$ and $A^{*}[12]$.

Let $\nabla: \Gamma\left(T M \oplus A^{*}\right) \times \Gamma(A) \rightarrow \Gamma(A)$ be the ordinary linear connection defined by $\nabla=\operatorname{pr}_{A} \circ \Delta \circ \iota_{A}$. The vector bundle map $l=\operatorname{pr}_{A}^{*}: A^{*} \rightarrow T M \oplus A^{*}$ is just the canonical inclusion. Define $\omega \in \Omega^{3}\left(T M \oplus A^{*}, A^{*}\right)$ by $\omega\left(v_{1}, v_{2}, v_{3}\right)=$ $\mathrm{Jac}_{\llbracket \cdot, \cdot \rrbracket}\left(v_{1}, v_{2}, v_{3}\right)$.

The objects $l, \llbracket \cdot, \cdot \rrbracket, \nabla^{*}, \omega$ define a split Lie 2-algebroid; the standard split Lie 2 -algebroid defined by the dull bracket (or equivalently by the dual Dorfman connection).

We give in $[9,10]$ the self-dual 2-representation $\left(\left(\rho, \rho^{*}\right): A \oplus T^{*} M \rightarrow T M \oplus\right.$ $\left.A^{*}, \nabla^{\text {bas }}, \nabla^{\text {bas }}, R_{\Delta}^{\text {bas }}\right)$ of $A$ that is defined by the VB-algebroid $\left(T A \oplus T^{*} A \rightarrow T M \oplus\right.$ $\left.A^{*}, A \rightarrow M\right)$ and any such Dorfman connection: The connections $\nabla^{\text {bas }}: \Gamma(A) \times$ $\Gamma\left(A \oplus T^{*} M\right) \rightarrow \Gamma\left(A \oplus T^{*} M\right)$ and $\nabla^{\text {bas }}: \Gamma(A) \times \Gamma\left(T M \oplus A^{*}\right) \rightarrow \Gamma\left(T M \oplus A^{*}\right)$ are

$$
\nabla_{a}^{\mathrm{bas}}(X, \alpha)=\left(\rho, \rho^{*}\right)\left(\Omega_{(X, \alpha)} a\right)+£_{a}(X, \alpha) \quad \text { and } \quad \nabla_{a}^{\mathrm{bas}}(b, \theta)=\Omega_{\left(\rho, \rho^{*}\right)(b, \theta)} a+£_{a}(b, \theta),
$$

where $\Omega: \Gamma\left(T M \oplus A^{*}\right) \times \Gamma(A) \rightarrow \Gamma\left(A \oplus T^{*} M\right)$ is defined by

$$
\Omega_{(X, \alpha)} a=\Delta_{(X, \alpha)}(a, 0)-(0, \mathbf{d}\langle\alpha, a\rangle)
$$

and for $a \in \Gamma(A)$, the derivations $£_{a}$ over $\rho(a)$ are defined by:

$$
£_{a}: \Gamma\left(A \oplus T^{*} M\right) \rightarrow \Gamma\left(A \oplus T^{*} M\right), \quad £_{a}(b, \theta)=\left([a, b], £_{\rho(a)} \theta\right)
$$

and

$$
£_{a}: \Gamma\left(T M \oplus A^{*}\right) \rightarrow \Gamma\left(T M \oplus A^{*}\right), \quad £_{a}(X, \alpha)=\left([\rho(a), X], £_{a} \alpha\right) .
$$


The basic curvature $R_{\Delta}^{\text {bas }}: \Gamma(A) \times \Gamma(A) \times \Gamma\left(T M \oplus A^{*}\right) \rightarrow \Gamma\left(A \oplus T^{*} M\right)$ is given by

$$
\begin{gathered}
R_{\Delta}^{\mathrm{bas}}(a, b)(X, \xi)=-\Omega_{(X, \xi)}[a, b]+£_{a}\left(\Omega_{(X, \xi)} b\right)-£_{b}\left(\Omega_{(X, \xi)} a\right) \\
+\Omega_{\nabla_{b}^{\mathrm{bas}}(X, \xi)} a-\Omega_{\nabla_{a}^{\mathrm{bas}}(X, \xi)} b .
\end{gathered}
$$

A straightforward computation, that also resembles much the one in [5, Section 3.2] for the tangent double of a Lie algebroid, shows that the Dorfman 2-representation and the self-dual 2-representation form a matched pair. Hence, $T A \oplus_{A} T^{*} A$ is an LA-Courant algebroid.

\subsubsection{The LA-Courant Algebroid Defined by a Double Lie Algebroid}

More generally, let

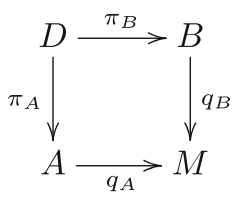

(with core $C$ ) be a double Lie algebroid. Then the pair $\left(D, D_{B}^{*}\right)$ of vector bundles over $B$ is a Lie bialgebroid, with $D_{B}^{*}$ endowed with the trivial Lie algebroid structure. We get a linear Courant algebroid $D \oplus_{B}\left(D_{B}^{*}\right)$ over $B$ with side $A \oplus C^{*}$

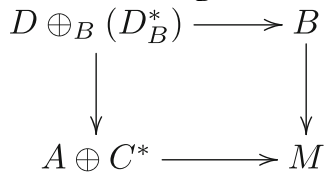

and core $C \oplus A^{*}$. The Courant algebroid structure is linear, see [12], Section 4.4.2. Recall also from there that a linear decomposition $\Sigma: A \times{ }_{M} B \times{ }_{M} C \rightarrow D$ defines a Lagrangian decomposition $\widetilde{\Sigma}$ of the metric double vector bundle $D \oplus_{B}\left(D_{B}^{*}\right)$. Further, the linear decomposition $\Sigma$ of $D$ yields a matched pair of 2-representations as in Section 2.4.

In the Lagrangian decomposition, the linear Courant algebroid structure is equivalent to the split Lie 2-algebroid $\left(\partial_{B} \circ \operatorname{pr}_{C}: C \oplus A^{*} \rightarrow B, \Delta, \nabla, R\right)$ defined by

$$
\begin{gathered}
\Delta: \quad \Gamma\left(A \oplus C^{*}\right) \times \Gamma\left(C \oplus A^{*}\right) \rightarrow \Gamma\left(C \oplus A^{*}\right) \\
\Delta_{(a, \gamma)}(c, \alpha)=\left(\nabla_{a} c, f_{a} \alpha+\left\langle\nabla_{\cdot}^{*} \gamma, c\right\rangle\right), \\
\nabla: \quad \Gamma\left(A \oplus C^{*}\right) \times \Gamma(B) \rightarrow \Gamma(B), \quad \nabla_{(a, \gamma)} b=\nabla_{a} b
\end{gathered}
$$

with $A \oplus C^{*}$ anchored by $\rho_{A}$, and $\omega \in \Omega^{3}\left(A \oplus C^{*}, B^{*}\right)$ defined by

$$
\mathbf{i}_{\left(a_{2}, \gamma_{2}\right)} \mathbf{i}_{\left(a_{1}, \gamma_{1}\right)} \omega=\left(R\left(a_{1}, a_{2}\right),\left\langle\gamma_{2}, R\left(a_{1}, \cdot\right)\right\rangle+\left\langle\gamma_{1}, R\left(\cdot, a_{2}\right)\right\rangle\right)
$$

as a section of $\operatorname{Hom}\left(B, C \oplus A^{*}\right)$.

The direct sum $D \oplus_{B} D_{B}^{*}$ over $B$ has also a VB-algebroid structure $\left(D \oplus_{B} D_{B}^{*} \rightarrow\right.$ $\left.A \oplus C^{*}, B \rightarrow M\right)$ with core $C \oplus A^{*}$. The linear decomposition $\tilde{\Sigma}: B \times_{M}(A \oplus$ $\left.C^{*}\right) \times_{M}\left(C \oplus A^{*}\right) \rightarrow D \oplus_{B}\left(D_{B}^{*}\right)$ defines the 2-representation of $B$

$$
\left(\partial_{A} \oplus \partial_{A}^{*}: C \oplus A^{*} \rightarrow A \oplus C^{*}, \nabla^{A} \oplus \nabla^{C^{*}}, \nabla^{C} \oplus \nabla^{A^{*}}, R \oplus\left(-R^{*}\right)\right),
$$


see [10], Section 4.5.2.

A straightforward computation shows that the matched pair conditions for the 2representations describing the sides of $D$ imply that the 2-representation (28) and the split Lie 2-algebroid (25)-(27) form a matched pair. Hence, $\left(D \oplus_{B}\left(D_{B}^{*}\right), A \oplus\right.$ $\left.C^{*}, B, M\right)$ has a natural LA-Courant algebroid structure. In the same manner, $\left(D \oplus_{A}\right.$ $\left.\left(D_{A}^{*}\right), B \oplus C^{*}, A, M\right)$ has a natural LA-Courant algebroid structure. Hence, we get the following theorem.

Theorem 3.8 Consider a matched pair of 2-representations with the usual notation. Then the split [2]-manifold $\left(A \oplus C^{*}\right)[-1] \oplus B^{*}[-2]$ endowed with the semi-direct Lie 2-algebroid structure in (25)-(27) and the Poisson bracket defined by (28), is a split Poisson Lie 2-algebroid.

By symmetry, the split [2]-manifold $\left(B \oplus C^{*}\right)[-1] \oplus A^{*}[-2]$ also inherits a split Poisson Lie 2-algebroid structure.

In the case of the double Lie algebroid $T A$, for $A \rightarrow M$ a Lie algebroid, the two LA-Courant algebroids obtained in this manner are $T A \oplus_{A} T^{*} A$ described in Section 3.3.2, and the tangent prolongation as in Section 3.3.1 of the Courant algebroid $A \oplus A^{*} \rightarrow M$; 【( $\left.a_{1}, \alpha_{1}\right),\left(a_{2}, \alpha_{2}\right) \rrbracket=\left(\left[a_{1}, a_{2}\right], £_{a_{1}} \alpha_{2}-\mathbf{i}_{a_{2}} \mathbf{d} \alpha_{1}\right)$ for $a_{1}, a_{2} \in \Gamma(A)$ and $\alpha_{1}, \alpha_{2} \in \Gamma\left(A^{*}\right)$.

\section{The Core of an LA-Courant Algebroid}

We prove in this section that the core of an LA-Courant algebroid inherits a natural structure of degenerate Courant algebroid. We discuss some examples and we deduce a new way of describing the equivalence between Courant algebroids and symplectic Lie 2-algebroids.

Theorem 4.1 Let $(\mathbb{E}, B, Q, M)$ be an LA-Courant algebroid and choose a Lagrangian splitting. Then the core $Q^{*}$ inherits the structure of a degenerate Courant algebroid over $M$, with the anchor $\rho_{Q^{*}}=\rho_{Q} \partial_{Q}$, the map $\mathcal{D}=\rho_{Q}^{*} \mathbf{d}: C^{\infty}(M) \rightarrow$ $\Gamma\left(Q^{*}\right)$, the pairing defined by $\left\langle\tau_{1}, \tau_{2}\right\rangle_{Q^{*}}=\left\langle\tau_{1}, \partial_{Q} \tau_{2}\right\rangle$ and the bracket defined by

$$
\llbracket \tau_{1}, \tau_{2} \rrbracket_{Q^{*}}=\Delta_{\partial_{Q} \tau_{1}} \tau_{2}-\nabla_{\partial_{B} \tau_{2}}^{*} \tau_{1}
$$

for all $\tau_{1}, \tau_{2} \in \Gamma\left(Q^{*}\right)$. This structure does not depend on the choice of the Lagrangian splitting, and the map $\partial_{B}: Q^{*} \rightarrow B$ is compatible with the brackets and the anchors: $\rho_{B} \partial_{B}=\rho_{Q^{*}}$ and

$$
\partial_{B} \llbracket \tau_{1}, \tau_{2} \rrbracket Q^{*}=\left[\partial_{B} \tau_{1}, \partial_{B} \tau_{2}\right]
$$

for all $\tau_{1}, \tau_{2} \in \Gamma\left(Q^{*}\right)$.

Proof Theorem 3.7 states that the 2-representation is self-dual and that the 2representation and the split Lie 2-algebroid defined by a Lagrangian splitting form a matched pair. Hence, by Section 2.5.1, the pairing $\langle\cdot, \cdot\rangle_{Q^{*}}$ is symmetric. The map 
$\rho_{Q}^{*} \mathbf{d}: C^{\infty}(M) \rightarrow \Gamma\left(Q^{*}\right)$ satisfies $\left\langle\tau, \rho_{Q}^{*} \mathbf{d} f\right\rangle_{Q^{*}}=\left\langle\partial_{Q} \tau, \rho_{Q}^{*} \mathbf{d} f\right\rangle=\left\langle\rho_{Q} \partial_{Q} \tau, \mathbf{d} f\right\rangle=$ $\left(\rho_{Q} \circ \partial_{Q}\right)(\tau)(f)$ for all $\tau \in \Gamma\left(Q^{*}\right)$ and $f \in C^{\infty}(M)$. We check (CA1)-(CA5) in the definition of a degenerate Courant algebroid (see Page 5). Condition (CA5) is immediate by definition of the bracket. Condition (CA3) is exactly (21). Note that (M2) and (12) imply

$$
\begin{aligned}
\partial_{B} \llbracket \tau_{1}, \tau_{2} \rrbracket Q^{*} & =\partial_{B}\left(\Delta_{\partial} \tau_{1} \tau_{2}-\nabla_{\partial_{B} \tau_{2}}^{*} \tau_{1}\right) \\
& =\nabla_{\partial_{Q} \tau_{1}} \partial_{B} \tau_{2}-\left[\partial_{B} \tau_{2}, \partial_{B} \tau_{1}\right]-\nabla_{\partial_{Q} \tau_{1}} \partial_{B} \tau_{2}=\left[\partial_{B} \tau_{1}, \partial_{B} \tau_{2}\right] .
\end{aligned}
$$

This and $\rho_{Q} \circ \partial_{Q}=\rho_{B} \circ \partial_{B}$ (see Remark 3.2) imply the last claim of the theorem. In the same manner (M1) and $\nabla \circ \partial_{Q}=\partial_{Q} \circ \nabla^{*}$ (by Definition of a 2-representation) imply the equation

$$
\partial_{Q} \llbracket \tau_{1}, \tau_{2} \rrbracket_{Q^{*}}=\llbracket \partial_{Q} \tau_{1}, \partial_{Q} \tau_{2} \rrbracket_{\Delta}+\partial_{B}^{*}\left\langle\tau_{2}, \nabla . \partial_{Q} \tau_{1}\right\rangle .
$$

The compatibility of the bracket with the anchor (CA4) follows then immediately from (30) with (11), or from (29) with $\rho_{Q} \circ \partial_{Q}=\rho_{B} \circ \partial_{B}$. Next we check (CA2) using (30) and $\nabla \circ \partial_{Q}=\partial_{Q} \circ \nabla^{*}$. We have

$$
\begin{gathered}
\rho_{Q} \partial_{Q}\left(\tau_{1}\right)\left\langle\tau_{2}, \tau_{3}\right\rangle_{Q^{*}}-\left\langle\llbracket \tau_{1}, \tau_{2} \rrbracket_{Q^{*}}, \tau_{3}\right\rangle_{Q^{*}}-\left\langle\tau_{2}, \llbracket \tau_{1}, \tau_{3} \rrbracket_{Q^{*}}\right\rangle_{Q^{*}} \\
=\left\langle\tau_{2}, \llbracket \partial_{Q} \tau_{1}, \partial_{Q} \tau_{3} \rrbracket_{\Delta}\right\rangle+\left\langle\nabla_{\partial_{B} \tau_{2}}^{*} \tau_{1}, \partial_{Q} \tau_{3}\right\rangle-\left\langle\tau_{2}, \partial_{Q} \llbracket \tau_{1}, \tau_{3} \rrbracket_{Q^{*}}\right\rangle=0
\end{gathered}
$$

since $\left\langle\nabla_{\partial_{B} \tau_{2}}^{*} \tau_{1}, \partial_{Q} \tau_{3}\right\rangle=\left\langle\partial_{Q} \nabla_{\partial_{B} \tau_{2}}^{*} \tau_{1}, \tau_{3}\right\rangle=\left\langle\nabla_{\partial_{B} \tau_{2}}^{*}\left(\partial_{Q} \tau_{1}\right), \tau_{3}\right\rangle$. Finally we check the Jacobi identity (CA1). Using (29) and (30), we have for $\tau_{1}, \tau_{2}, \tau_{3} \in \Gamma\left(Q^{*}\right)$ :

$$
\begin{aligned}
& \llbracket \llbracket \tau_{1}, \tau_{2} \rrbracket Q^{*}, \tau_{3} \rrbracket Q^{*}+\llbracket \tau_{2}, \llbracket \tau_{1}, \tau_{3} \rrbracket Q^{*} \rrbracket Q^{*}-\llbracket \tau_{1}, \llbracket \tau_{2}, \tau_{3} \rrbracket Q^{*} \rrbracket Q^{*} \\
& =\Delta_{\llbracket \partial_{Q} \tau_{1}, \partial_{Q} \tau_{2} \rrbracket+\partial_{B}^{*}\left\langle\tau_{2}, \nabla . \partial_{Q} \tau_{1}\right\rangle} \tau_{3}-\nabla_{\partial_{B} \tau_{3}}^{*}\left(\Delta_{\partial_{Q} \tau_{1}} \tau_{2}-\nabla_{\partial_{B} \tau_{2}}^{*} \tau_{1}\right) \\
& +\Delta_{\partial_{Q} \tau_{2}}\left(\Delta_{\partial_{Q} \tau_{1}} \tau_{3}-\nabla_{\partial_{B} \tau_{3}}^{*} \tau_{1}\right)-\nabla_{\left[\partial_{B} \tau_{1}, \partial_{B} \tau_{3}\right]}^{*} \tau_{2} \\
& -\Delta_{\partial_{Q} \tau_{1}}\left(\Delta_{\partial Q} \tau_{2} \tau_{3}-\nabla_{\partial_{B} \tau_{3}}^{*} \tau_{2}\right)+\nabla_{\left[\partial_{B} \tau_{2}, \partial_{B} \tau_{3}\right]}^{*} \tau_{1} \\
& =R_{\nabla}\left(\partial_{B} \tau_{3}, \partial_{B} \tau_{2}\right) \tau_{1}+\nabla_{\partial_{B} \tau_{2}}^{*} \nabla_{\partial_{B} \tau_{3}}^{*} \tau_{1}-R_{\Delta}\left(\partial_{Q} \tau_{1}, \partial_{Q} \tau_{2}\right) \tau_{3}+\Delta_{\partial_{B}^{*}\left\langle\tau_{2}, \nabla . \partial_{Q} \tau_{1}\right\rangle} \tau_{3} \\
& -\nabla_{\partial_{B} \tau_{3}}^{*} \Delta_{\partial_{Q} \tau_{1}} \tau_{2}+\Delta_{\partial_{Q} \tau_{1}} \nabla_{\partial_{B} \tau_{3}}^{*} \tau_{2}-\Delta_{\partial_{Q} \tau_{2}} \nabla_{\partial_{B} \tau_{3}}^{*} \tau_{1}-\nabla_{\left[\partial_{B} \tau_{1}, \partial_{B} \tau_{3}\right]}^{*} \tau_{2} .
\end{aligned}
$$

Using the equalities $R_{\Delta}\left(\partial_{Q} \tau_{1}, \partial_{Q} \tau_{2}\right) \tau_{3}=\left\langle\mathbf{i}_{\partial_{Q} \tau_{2}} \mathbf{i}_{\partial Q} \tau_{1} \omega, \partial_{B} \tau_{3}\right\rangle$ by (13), $R_{\nabla}\left(\partial_{B} \tau_{3}, \partial_{B} \tau_{2}\right) \tau_{1}=R\left(\partial_{B} \tau_{3}, \partial_{B} \tau_{2}\right) \partial_{Q} \tau_{1}$ by the definition of a 2-representation, and (22), this is

$$
\begin{aligned}
& -\Delta_{\nabla_{\partial_{B} \tau_{3}} \partial_{Q} \tau_{1}} \tau_{2}+\nabla_{\nabla_{\partial_{Q} \tau_{1} \partial_{B} \tau_{3}}^{*}} \tau_{2}+\left\langle\nabla_{\nabla . \partial_{B} \tau_{3}} \partial_{Q} \tau_{1}, \tau_{2}\right\rangle \\
& \quad+\nabla_{\partial_{B} \tau_{2}}^{*} \nabla_{\partial_{B} \tau_{3}}^{*} \tau_{1}+\Delta_{\partial_{B}^{*}\left\langle\tau_{2}, \nabla . \partial_{Q} \tau_{1}\right\rangle} \tau_{3}-\nabla_{\left[\partial_{B} \tau_{1}, \partial_{B} \tau_{3}\right]}^{*} \tau_{2}-\Delta_{\partial_{Q} \tau_{2}} \nabla_{\partial_{B} \tau_{3}}^{*} \tau_{1} .
\end{aligned}
$$

By (21), we can replace

$$
\begin{aligned}
& -\Delta_{\nabla_{\partial_{B} \tau_{3}} \partial_{Q} \tau_{1}} \tau_{2}+\nabla_{\partial_{B} \tau_{2}}^{*} \nabla_{\partial_{B} \tau_{3}}^{*} \tau_{1}-\Delta_{\partial_{Q} \tau_{2}} \nabla_{\partial_{B} \tau_{3}}^{*} \tau_{1} \\
& =-\Delta_{\partial_{Q}\left(\nabla_{\partial_{B} \tau_{3}}^{*} \tau_{1}\right)} \tau_{2}+\nabla_{\partial_{B} \tau_{2}}^{*}\left(\nabla_{\partial_{B} \tau_{3}}^{*} \tau_{1}\right)-\Delta_{\partial_{Q} \tau_{2}}\left(\nabla_{\partial_{B} \tau_{3}}^{*} \tau_{1}\right)
\end{aligned}
$$

by

$$
-\nabla_{\partial_{B}\left(\nabla_{\partial_{B} \tau_{3}}^{*} \tau_{1}\right)}^{*} \tau_{2}-\rho_{Q}^{*} \mathbf{d}\left\langle\tau_{2}, \partial_{Q} \nabla_{\partial_{B} \tau_{3}}^{*} \tau_{1}\right\rangle
$$


and we get

$$
\begin{aligned}
& \nabla_{\nabla_{\partial} \mathcal{Q}_{1} \partial_{B} \tau_{3}-\left[\partial_{B} \tau_{1}, \partial_{B} \tau_{3}\right]-\partial_{B}\left(\nabla_{\partial_{B} \tau_{3}} \tau_{1}\right)}^{*} \tau_{2}+\left\langle\nabla_{\nabla . \partial_{B} \tau_{3}} \partial_{Q} \tau_{1}, \tau_{2}\right\rangle \\
& \quad+\Delta_{\partial_{B}^{*}}\left\langle\tau_{2}, \nabla . \partial_{Q} \tau_{1}\right\rangle \tau_{3}-\rho_{Q}^{*} \mathbf{d}\left\langle\tau_{2}, \partial_{Q} \nabla_{\partial_{B} \tau_{3}}^{*} \tau_{1}\right\rangle
\end{aligned}
$$

Since $\nabla_{\partial_{Q} \tau_{1}} \partial_{B} \tau_{3}-\left[\partial_{B} \tau_{1}, \partial_{B} \tau_{3}\right]-\partial_{B}\left(\nabla_{\partial_{B} \tau_{3}} \tau_{1}\right)=0$ by (M2), we finally get

$$
\left\langle\nabla_{\nabla . \partial_{B} \tau_{3}} \partial_{Q} \tau_{1}, \tau_{2}\right\rangle+\Delta_{\partial_{B}^{*}\left\langle\tau_{2}, \nabla . \partial_{Q} \tau_{1}\right\rangle} \tau_{3}-\rho_{Q}^{*} \mathbf{d}\left\langle\tau_{2}, \partial_{Q} \nabla_{\partial_{B}} \tau_{3} \tau_{1}\right\rangle .
$$

We write $\beta:=\left\langle\nabla . \partial_{Q} \tau_{1}, \tau_{2}\right\rangle \in \Gamma\left(B^{*}\right)$. Since $\rho_{Q} \circ \partial_{Q}=\rho_{B} \circ \partial_{B}$ and $\nabla \circ \partial_{Q}=\partial_{Q} \circ \nabla$, we find $\beta=\left\langle\partial_{Q} \nabla^{*} \tau_{1}, \tau_{2}\right\rangle=\left\langle\nabla^{*} \tau_{1}, \partial_{Q} \tau_{2}\right\rangle \in \Gamma\left(B^{*}\right)$. To see that (31), which is a section of $Q^{*}$, vanishes, we evaluate it on an arbitrary $q \in \Gamma(Q)$. We use (12) and the definition of a 2-representation and we get

$$
\begin{aligned}
& \left\langle\nabla_{\nabla_{q} \partial_{B} \tau_{3}} \partial_{Q} \tau_{1}, \tau_{2}\right\rangle+\left\langle\Delta_{\partial_{B}^{*} \beta} \tau_{3}, q\right\rangle-\rho_{Q}(q)\left\langle\tau_{2}, \partial_{Q} \nabla_{\partial_{B} \tau_{3}}^{*} \tau_{1}\right\rangle \\
= & \left\langle\nabla_{\partial_{B} \Delta_{q} \tau_{3}} \partial_{Q} \tau_{1}, \tau_{2}\right\rangle+\left\langle\Delta_{\partial_{B}^{*} \beta} \tau_{3}, q\right\rangle-\rho_{Q}(q)\left\langle\tau_{2}, \partial_{Q} \nabla_{\partial_{B} \tau_{3}}^{*} \tau_{1}\right\rangle \\
= & \left\langle\beta, \partial_{B} \Delta_{q} \tau_{3}\right\rangle+\left\langle\Delta_{\partial_{B}^{*} \beta} \tau_{3}, q\right\rangle-\rho_{Q}(q)\left\langle\beta, \partial_{B} \tau_{3}\right\rangle=-\left\langle\llbracket q, \partial_{B}^{*} \beta \rrbracket_{\Delta}, \tau_{3}\right\rangle+\left\langle\Delta_{\partial_{B}^{*} \beta} \tau_{3}, q\right\rangle .
\end{aligned}
$$

Since the Dorfman connection $\Delta$ is dual to the skew-symmetric dull bracket $\llbracket \cdot \cdot, \rrbracket_{\Delta}$, this is $\rho_{Q}\left(\partial_{B}^{*} \beta\right)\left\langle q, \tau_{3}\right\rangle$. Because $\rho_{Q} \circ \partial_{B}^{*}=0$ by (11), we can conclude.

We finally prove that the degenerate Courant algebroid structure does not depend on the choice of the Lagrangian splitting. Clearly the pairing and anchor are independent of the splitting, so we only need to check that the bracket remains the same if we choose a different Lagrangian splitting. Assume that $\Sigma_{1}, \Sigma_{2}: B \times_{M} Q \times_{M} Q^{*} \rightarrow \mathbb{E}$ are two Lagrangian decompositions. Then there is $\phi \in \Gamma\left(B^{*} \otimes Q^{*} \wedge Q^{*}\right)$ such that for all $\left(b_{m}, q_{m}, \tau_{m}\right) \in B \times \times_{M} Q \times_{M} Q^{*}, \Sigma_{1}\left(b_{m}, q_{m}, \tau_{m}\right)=\Sigma_{2}\left(b_{m}, q_{m}, \tau_{m}\right){ }_{B}$ $\left(0_{b_{m}}^{\mathbb{E}}+{ }_{Q} \phi\left(b_{m}, q_{m}\right)\right)$. Then by Remark 2.12 of [5], we have $\nabla_{b}^{2} \tau=\nabla_{b}^{1} \tau+\phi\left(b, \partial_{Q} \tau\right)$ for all $b \in \Gamma(B)$ and $\tau \in \Gamma\left(Q^{*}\right)$. By Proposition 4.7 in [12], we have $\Delta_{q}^{2} \tau=$ $\Delta_{q}^{1} \tau+\phi\left(\partial_{B} \tau, q\right)$ for all $q \in \Gamma(Q)$ and $\tau \in \Gamma\left(Q^{*}\right)$. Then $\Delta_{\partial_{Q} \tau_{1}}^{2} \tau_{2}-\nabla_{\partial_{B} \tau_{2}}^{2} \tau_{1}=$ $\Delta_{\partial_{Q} \tau_{1}}^{1} \tau_{2}+\phi\left(\partial_{B} \tau_{2}, \partial_{Q} \tau_{1}\right)-\nabla_{\partial_{B} \tau_{2}}^{1} \tau_{1}-\phi\left(\partial_{B} \tau_{2}, \partial_{Q} \tau_{1}\right)=\Delta_{\partial_{Q} \tau_{1}}^{1} \tau_{2}-\nabla_{\partial_{B} \tau_{2}}^{1} \tau_{1}$.

Example 4.2 (Tangent Courant algebroid) Consider the example described in Section 3.3.1. The degenerate Courant algebroid structure on the core $E$ of $T E$ is just the initial Courant algebroid structure on $\mathrm{E}$ since $\Delta_{e_{1}} e_{2}=\llbracket e_{1}, e_{2} \rrbracket+\nabla_{\rho\left(e_{2}\right)} e_{1}$ by definition and so

$$
\Delta_{e_{1}} e_{2}-\nabla_{\rho\left(e_{2}\right)} e_{1}=\llbracket e_{1}, e_{2} \rrbracket .
$$

We have hence proved that the Courant algebroid associated to a symplectic Lie 2-algebroid can be defined directly from any of the splittings of the Lie 2-algebroid, and so does not need to be obtained as a derived bracket.

Theorem 4.3 Let $\mathcal{M}$ be a symplectic Lie 2-algebroid over a base manifold $M$. Then the corresponding Courant algebroid is defined as follows. Choose any splitting $\mathcal{M} \simeq Q[-1] \oplus T^{*} M[-2]$ of the underlying symplectic [2]-manifold. Then $Q \simeq Q^{*}$ via $\partial_{Q}$ and $Q^{*}$ inherits a nondegenerate pairing given by $\left\langle\tau_{1}, \partial_{Q} \tau_{2}\right\rangle$ for $\tau_{1}, \tau_{2} \in \Gamma\left(Q^{*}\right)$. The morphism $\partial_{T M}: Q^{*} \rightarrow T M$ of the split Lie 2-algebroid structure on $Q[-1] \oplus T^{*} M[-2]$ defines an anchor on $Q^{*}$. We have further a bracket 
$\llbracket \cdot, \cdot \rrbracket Q^{*}$ defined on $\Gamma\left(Q^{*}\right)$ by $\llbracket \tau_{1}, \tau_{2} \rrbracket Q^{*}=\Delta_{\partial_{Q} \tau_{1}} \tau_{2}-\left\{\partial_{T M} \tau_{2}, \tau_{1}\right\}$ and that does not depend on the choice of the splitting. This anchor, pairing and bracket define a Courant algebroid structure on $Q^{*}$.

Note that the Courant algebroid structure is transported to $Q$ by $\boldsymbol{\beta}=\partial_{Q}: Q^{*} \rightarrow$ $Q$ for our result to be consistent with the construction in Section 3.3.1.

Example 4.4 (Core of the standard Courant algebroid over a Lie algebroid) Consider now the example discussed in Section 3.3.2; namely the standard LA-Courant algebroid

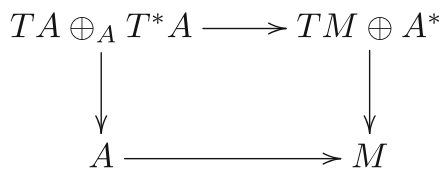

over a Lie algebroid $A$. The degenerate Courant algebroid structure on the core $A \oplus$ $T^{*} M$ of $T A \oplus T^{*} A$ is here given by $\rho_{A \oplus T^{*} M}(a, \theta)=\rho_{A}(a)$,

$$
\left\langle\left(a_{1}, \theta_{1}\right),\left(a_{2}, \theta_{2}\right)\right\rangle_{A \oplus T^{*} M}=\left\langle\left(a_{1}, \theta_{1}\right),\left(\rho_{A}, \rho_{A}^{*}\right)\left(a_{2}, \theta_{2}\right)\right\rangle
$$

and the bracket defined by

$$
\llbracket\left(a_{1}, \theta_{1}\right),\left(a_{2}, \theta_{2}\right) \rrbracket_{A \oplus T^{*} M}=\left(\left[a_{1}, a_{2}\right], £_{\rho_{A}\left(a_{1}\right)} \theta_{2}-\mathbf{i}_{\rho_{A}\left(a_{2}\right)} \mathbf{d} \theta_{1}\right)
$$

for all $a, a_{1}, a_{2} \in \Gamma(A)$ and $\theta, \theta_{1}, \theta_{2} \in \Omega^{1}(M)$. To see this, use Lemma 5.16 in [9] or the next example; this degenerate Courant algebroid plays a crucial role in the infinitesimal description of Dirac groupoids [11], i.e. in the definition of Dirac bialgebroids.

Example 4.5 (LA-Courant algebroid associated to a double Lie algebroid) More generally, the LA-Courant algebroids (and the corresponding Poisson Lie 2-algebroids) considered in Section 3.3.3 and Theorem 4.1 yield the following application.

A matched pair of 2-representations as in Section 2.4 defines two degenerate Courant algebroids. The first one is $C \oplus A^{*} \rightarrow M$ with the anchor $\rho_{C \oplus A^{*}}: C \oplus A^{*} \rightarrow$ $T M$ defined by $\rho_{C \oplus A^{*}}=\rho_{A} \circ \operatorname{pr}_{A} \circ\left(\partial_{A} \oplus \partial_{A}^{*}\right)=\rho_{B} \circ \partial_{B} \circ \operatorname{pr}_{C}$. The pairing is defined by

$$
\left\langle\left(c_{1}, \alpha_{1}\right),\left(c_{2}, \alpha_{2}\right)\right\rangle_{C \oplus A^{*}}=\left\langle\alpha_{1}, \partial_{A} c_{2}\right\rangle+\left\langle\alpha_{2}, \partial_{A} c_{1}\right\rangle
$$

for all $\alpha_{1}, \alpha_{2} \in \Gamma\left(A^{*}\right)$ and $c_{1}, c_{2} \in \Gamma(C)$, and the bracket by

$$
\begin{aligned}
\llbracket\left(c_{1}, \alpha_{1}\right),\left(c_{2}, \alpha_{2}\right) \rrbracket_{C \oplus A^{*}} & =\Delta_{\left(\partial_{A} c_{1}, \partial_{A}^{*} \alpha_{1}\right)}\left(c_{2}, \alpha_{2}\right)-\nabla_{\partial_{B} c_{2}}\left(c_{1}, \alpha_{1}\right) \\
& =\left(\nabla_{\partial_{A} c_{1}} c_{2}-\nabla_{\partial_{B} c_{2}} c_{1}, £_{\partial_{A} c_{1}} \alpha_{2}+\left\langle\nabla^{*} \partial_{A}^{*} \alpha_{1}, c_{2}\right\rangle-\nabla_{\partial_{B} c_{2}}^{*} \alpha_{1}\right) \\
& =\left(\left[c_{1}, c_{2}\right], £_{\partial_{A} c_{1}} \alpha_{2}-\mathbf{i}_{\partial_{A} c_{2}} \mathbf{d}_{A} \alpha_{1}\right) .
\end{aligned}
$$

Note that the restriction to $\Gamma(C)$ of the Courant bracket is the Lie algebroid bracket induced on $C$ by the matched pair, see Section 2.4.

The second degenerate Courant algebroid is $C \oplus B^{*} \rightarrow M$ with the anchor $\rho_{C \oplus B^{*}}: C \oplus B^{*} \rightarrow T M$ defined by $\rho_{B} \circ \operatorname{pr}_{B} \circ\left(\partial_{B} \oplus \partial_{B}^{*}\right)=\rho_{B} \circ \partial_{B} \circ \operatorname{pr}_{C}$, the pairing 
defined by

$$
\left\langle\left(c_{1}, \beta_{1}\right),\left(c_{2}, \beta_{2}\right)\right\rangle_{C \oplus B^{*}}=\left\langle\beta_{1}, \partial_{B} c_{2}\right\rangle+\left\langle\beta_{2}, \partial_{B} c_{1}\right\rangle
$$

for all $\beta_{1}, \beta_{2} \in \Gamma\left(B^{*}\right)$ and $c_{1}, c_{2} \in \Gamma(C)$, and the bracket

$$
\llbracket\left(c_{1}, \beta_{1}\right),\left(c_{2}, \beta_{2}\right) \rrbracket_{C \oplus B^{*}}=\left(\nabla_{\partial_{B} c_{1}} c_{2}-\nabla_{\partial_{A} c_{2}} c_{1}, £_{\partial_{B} c_{1}} \beta_{2}-\mathbf{i}_{\partial_{B} c_{2}} \mathbf{d}_{B} \beta_{1}\right) .
$$

Here again, by $(\mathrm{m} 1)$, the restriction to $\Gamma(C)$ of the Courant bracket is the Lie algebroid bracket induced on $C$ by the matched pair, as in Section 2.4.

\section{VB-Dirac Structures, LA-Dirac Structures and Pseudo Dirac Structures}

In this section, we study isotropic subalgebroids of metric VB-algebroids and Dirac structures in VB- and LA-Courant algebroids. While we paid attention in the preceding sections to bridge [2]-geometric objects to geometric structures on metric double vector bundles, we are here more interested in classifications of VB-Dirac structures via the simple geometric descriptions that we found before for VB-Courant algebroids and LA-Courant algebroids.

\subsection{VB-Dirac Structures}

Let $(\mathbb{E}, B, Q, M)$ be a VB-Courant algebroid with core $Q^{*}$ and anchor $\Theta: \mathbb{E} \rightarrow T B$. Let $D$ be a double vector subbundle structure over $B^{\prime} \subseteq B$ and $U \subseteq Q$ and with core $K$. Choose a linear splitting $\Sigma: B \times{ }_{M} Q \rightarrow \mathbb{E}$ that is adapted ${ }^{5}$ to $D$, i.e. such that $\Sigma\left(B^{\prime} \times_{M} U\right) \subseteq D$. Then $D$ is spanned as a vector bundle over $B^{\prime}$ by the sections $\left.\sigma_{Q}(u)\right|_{B^{\prime}}$ for all $u \in \Gamma(U)$ and $\left.\tau^{\dagger}\right|_{B^{\prime}}$ for all $\tau \in \Gamma(K)$.

We get immediately the following proposition.

Proposition 5.1 In the situation described above, the double subbundle $D \subseteq \mathbb{E}$ over $B^{\prime}$ is isotropic if and only if $K \subseteq U^{\circ}$ and $\Lambda$ as in (5) sends $U \otimes U$ to $B^{\prime \circ}$.

Proposition 5.2 In the situation described above, $D$ is maximal isotropic if and only if $U=K^{\circ}$ and $\Lambda$ sends $U \otimes U$ to $B^{\prime \circ}$.

\footnotetext{
${ }^{5}$ Since $D$ and $\mathbb{E}$ are both double vector bundles, there exist two decompositions $\mathbb{I}_{D}: B^{\prime} \times_{M} U \times{ }_{M} K \rightarrow D$ and $\mathbb{I}: B \times{ }_{M} Q \times_{M} Q^{*} \rightarrow \mathbb{E}$. Let $\iota: D \rightarrow \mathbb{E}$ be the double vector bundle inclusion, over $\iota_{U}: U \rightarrow Q$ and $\iota_{B^{\prime}}: B^{\prime} \rightarrow B$, and with core morphism $\iota_{K}: K \rightarrow Q^{*}$. Then the map $\mathbb{I}^{-1} \circ \iota \circ \mathbb{I}_{D}: B^{\prime} \times_{M} U \times_{M}$ $K \rightarrow B \times_{M} Q \times_{M} Q^{*}$ defines a morphism $\phi \in \Gamma\left(B^{\prime *} \otimes U^{*} \otimes Q^{*}\right)$ by $\left(\mathbb{I}^{-1} \circ \iota \circ \mathbb{I}_{D}\right)\left(b_{m}, u_{m}, k_{m}\right)=$ $\left(\iota_{B}\left(b_{m}\right), \iota_{U}\left(u_{m}\right), \iota_{K}\left(k_{m}\right)+\phi\left(b_{m}, u_{m}\right)\right)$. Using local basis sections of $B$ and $Q$ adapted to $B^{\prime}$ and $U$ and a partition of unity on $M$, extend $\phi$ to $\hat{\phi} \in \Gamma\left(B^{*} \otimes Q \otimes Q^{*}\right)$. Then define a new decomposition $\tilde{\mathbb{I}}^{-1}: \mathbb{E} \rightarrow$ $B \times_{M} Q \times_{M} Q^{*}$ by $\tilde{\mathbb{I}}^{-1}(e)=\mathbb{I}^{-1}(e)+_{B}\left(b_{m}, 0_{m}^{Q},-\hat{\phi}\left(b_{m}, q_{m}\right)\right)=\mathbb{I}^{-1}(e)+_{Q}\left(0_{m}^{B}, q_{m},-\hat{\phi}\left(b_{m}, q_{m}\right)\right)$ for $e \in \mathbb{E}$ with $\pi_{B}(e)=b_{m}$ and $\pi_{Q}(e)=q_{m}$. Then $\left(\tilde{\mathbb{I}}^{-1} \circ \iota \mathbb{I}_{D}\right)\left(b_{m}, u_{m}, k_{m}\right)=\left(\iota_{B}\left(b_{m}\right), \iota_{U}\left(u_{m}\right), \iota_{K}\left(k_{m}\right)\right)$ for all $\left(b_{m}, u_{m}, k_{m}\right) \in B^{\prime} \times_{M} U \times_{M} K$. The corresponding linear splitting $\tilde{\Sigma}: B \times_{M} Q \rightarrow \mathbb{E}, \tilde{\Sigma}\left(b_{m}, q_{m}\right)=$ $\tilde{\mathbb{I}}\left(b_{m}, q_{m}, 0_{m}^{Q^{*}}\right)$ sends $\left(\iota_{B^{\prime}}\left(b_{m}\right), \iota_{U}\left(u_{m}\right)\right)$ to $\iota\left(\mathbb{I}_{D}\left(b_{m}, u_{m}, 0_{m}^{K}\right) \in \iota(D)\right.$.
} 
Now we can prove that if $D$ is maximal isotropic, then there exists a Lagrangian splitting of $\mathbb{E}$ that is adapted to $D$.

Corollary 5.3 Let $(\mathbb{E}, B, Q, M)$ be a metric double vector bundle and $D \subseteq \mathbb{E} a$ maximal isotropic double subbundle. Then there exists a Lagrangian splitting that is adapted to $D$.

Proof As before, let $U \subseteq Q$ and $B^{\prime} \subseteq B$ be the sides of $D$. Then by Proposition 5.2 the core of $D$ is the vector bundle $U^{\circ} \subseteq Q^{*}$. Choose a linear splitting $\Sigma: Q \times{ }_{M} B \rightarrow$ $\mathbb{E}$ that is adapted to $D$. Then $D$ is spanned as a vector bundle over $B^{\prime}$ by the sections $\left.\sigma_{Q}(u)\right|_{B^{\prime}}$ for all $u \in \Gamma(U)$ and $\left.\tau^{\dagger}\right|_{B^{\prime}}$ for all $\tau \in \Gamma\left(U^{\circ}\right)$. As in (6), transform $\Sigma$ into a new Lagrangian linear splitting $\Sigma^{\prime}$. We need to show that $\left.\sigma_{Q}^{\prime}(u)\right|_{B^{\prime}}-\left.\sigma_{Q}(u)\right|_{B^{\prime}}$ is equivalent to a section of $B^{\prime *} \otimes U^{\circ}$ for all $u \in \Gamma(U)$. But $\sigma_{Q}^{\prime}(u)-\sigma_{Q}(u)=\widetilde{\frac{1}{2}} \widetilde{\Lambda(u, \cdot)}$ by construction and, since $D$ is isotropic, we have $\left.\Lambda\left(u, \widetilde{u^{\prime}}\right)\right|_{B^{\prime}}=0$ for all $u, u^{\prime} \in$ $\Gamma(U)$.

Remark 5.4 Consider a Courant algebroid E $\rightarrow M$ and its tangent double $T$ E. Recall from Example 2.2 that Lagrangian splittings of $T E$ are equivalent to metric connections $\mathfrak{X}(M) \times \Gamma(\mathrm{E}) \rightarrow \Gamma(\mathrm{E})$. Let $\nabla$ be such a metric connection, that is adapted to a maximally isotropic double subbundle $D$ over the sides $T M$ and $U \subseteq \mathrm{E}$. Define $[\nabla]: \mathfrak{X}(M) \times \Gamma(U) \rightarrow \Gamma\left(\mathrm{E} / U^{\perp}\right)$ by $[\nabla]_{X} u=\overline{\nabla_{X} u} \in \Gamma\left(\mathrm{E} / U^{\perp}\right)$. A second metric connection $\nabla^{\prime}: \mathfrak{X}(M) \times \Gamma(\mathrm{E}) \rightarrow \Gamma(\mathrm{E})$ is adapted to $D$ if and only if $\nabla_{X} u-\nabla_{X}^{\prime} u \in \Gamma\left(U^{\perp}\right)$ for all $X \in \mathfrak{X}(M)$ and for all $u \in \Gamma(U)$. Hence, if and only if $[\nabla]=\left[\nabla^{\prime}\right]$. We call $[\nabla]$ the invariant part of the metric connection adapted to $D$.

The existence of Lagrangian splittings of $\mathbb{E}$ adapted to maximal isotropic double subbundles $D$ will now be used to study the involutivity of $D$.

Note that in a very early version of this work, we studied VB-Courant algebroids via general (not necessarily Lagrangian) linear splittings. We found some more general objects than split Lie 2-algebroids; involving also $\Lambda \in S^{2}\left(Q, B^{*}\right)$ defined in (5). The study of the involutivity of general (not necessarily isotropic) double subbundles $D$ of $\mathbb{E}$ is therefore also possible in this more general framework, and yields very similar results.

Proposition 5.5 Let $(\mathbb{E}, B, Q, M)$ be a $V B$-Courant algebroid and $D \subseteq \mathbb{E}$ a maximal isotropic double subbundle. Choose a Lagrangian splitting of $\mathbb{E}$ that is adapted to $D$ and consider the corresponding split Lie 2-algebroid, denoted as usual. Then D is a Dirac structure in $\mathbb{E}$ with support $B^{\prime}$ if and only if

(1) $\partial_{B}\left(U^{\circ}\right) \subseteq B^{\prime}$,

(2) $\nabla_{u} b \in \Gamma\left(B^{\prime}\right)$ for all $u \in \Gamma(U)$ and $b \in \Gamma\left(B^{\prime}\right)$,

(3) $\llbracket u_{1}, u_{2} \rrbracket \in \Gamma(U)$ for all $u_{1}, u_{2} \in \Gamma(U)$,

(4) $\mathbf{i}_{u_{2}} \mathbf{i}_{u_{1}} \omega$ restricts to a section of $\Gamma\left(\operatorname{Hom}\left(B^{\prime}, U^{\circ}\right)\right)$ for all $u_{1}, u_{2} \in \Gamma(U)$.

A Dirac double subbundle $D$ of a VB-Courant algebroid $\mathbb{E}$ as in the proposition is called a VB-Dirac structure. 
Proof This is easy to prove using Lemma 3.5 on sections $\sigma_{Q}(u)$ and $\tau^{\dagger}$, for $u \in \Gamma(U)$ and $\tau \in \Gamma\left(U^{\circ}\right)$. Their anchors and Courant brackets can be described by

$$
\begin{aligned}
\Theta\left(\sigma_{Q}(u)\right) & =\widehat{\nabla_{u}} \in \mathfrak{X}^{l}(B), \quad \Theta\left(\tau^{\dagger}\right)=\left(\partial_{B} \tau\right)^{\uparrow} \in \mathfrak{X}^{c}(B), \\
\llbracket \sigma_{Q}\left(u_{1}\right), \sigma_{Q}\left(u_{2}\right) \rrbracket & =\sigma_{Q}\left(\llbracket u_{1}, u_{2} \rrbracket\right)-{\widehat{\mathbf{i}_{u_{2}} \mathbf{i}_{u_{1}}} \omega,}^{\Theta}, \\
\llbracket \sigma_{Q}(u), \tau^{\dagger} \rrbracket & =\left(\Delta_{u} \tau\right)^{\dagger}, \quad \llbracket \tau_{1}^{\dagger}, \tau_{2}^{\dagger} \rrbracket=0
\end{aligned}
$$

for all $u, u_{1}, u_{2} \in \Gamma(U)$ and $\tau, \tau_{1}, \tau_{2} \in \Gamma\left(U^{\circ}\right)$. The vector field $\widehat{\nabla_{u}}$ is tangent to $B^{\prime}$ on $B^{\prime}$ if and only if for all $\beta \in \Gamma\left(\left(B^{\prime}\right)^{\circ}\right), \widehat{\nabla_{u}}\left(\ell_{\beta}\right)=\ell_{\nabla_{u}^{*} \beta}$ vanishes on $B^{\prime}$. That is, if and only if, for all $\beta \in \Gamma\left(\left(B^{\prime}\right)^{\circ}\right), \nabla_{u}^{*} \beta$ is again a section of $\left(B^{\prime}\right)^{\circ}$. This yields (2). The vector field $\left(\partial_{B} \tau\right)^{\uparrow}$ is tangent to $B^{\prime}$ if and only if $\partial_{B} \tau \in \Gamma\left(B^{\prime}\right)$. This yields (1). Next, $\llbracket \sigma_{Q}(u), \tau^{\dagger} \rrbracket=\left(\Delta_{u} \tau\right)^{\dagger}$ is a section of $D$ over $B^{\prime}$ if and only if $\Delta_{u} \tau \in \Gamma\left(U^{\circ}\right)$. Since $\Delta_{u} \tau \in \Gamma\left(U^{\circ}\right)$ for all $u \in \Gamma(U)$ and $\tau \in \Gamma\left(U^{\circ}\right)$ if and only if $\llbracket u_{1}, u_{2} \rrbracket \in \Gamma(U)$ for all $u_{1}, u_{2} \in \Gamma(U)$, this is (3). Further, $\sigma_{Q}\left(\llbracket u_{1}, u_{2} \rrbracket\right)$ takes then values in $D$ over $B^{\prime}$, and so $\llbracket \sigma_{Q}\left(u_{1}\right), \sigma_{Q}\left(u_{2}\right) \rrbracket$ takes values in $D$ over $B^{\prime}$ if and only if $\mathbf{i}_{u_{2}} \mathbf{i}_{u_{1}} \omega$ restricts to a morphism $B^{\prime} \rightarrow U^{\circ}$. This is (4).

We get the following result for VB-Dirac structures (with support $B$ ) in $\mathbb{E}$.

Corollary 5.6 Let $(\mathbb{E}, B, Q, M)$ be a VB-Courant algebroid and $(D, B, U, M) \subseteq$ $\mathbb{E}$ a maximal isotropic double subbundle. Choose a Lagrangian splitting of $\mathbb{E}$ that is adapted to $D$ and consider the corresponding split Lie 2-algebroid, denoted as usual. If $D$ is a Dirac structure in $\mathbb{E} \rightarrow B$, then $U$ inherits a Lie algebroid structure with bracket $\llbracket \cdot, \cdot \rrbracket \mid \Gamma_{\Gamma}(U) \times \Gamma(U)$ and anchor $\left.\rho_{Q}\right|_{U}$. This Lie algebroid structure does not depend on the choice of Lagrangian splitting.

Proof By (3) in Proposition 5.5, $\llbracket \cdot, \cdot \rrbracket$ restricts to a bracket on sections of $U$. For $u_{1}, u_{2}, u_{3}, \mathrm{Jac}_{\llbracket \cdot, \cdot \rrbracket}\left(u_{1}, u_{2}, u_{3}\right)=\partial_{B}^{*} \omega\left(u_{1}, u_{2}, u_{3}\right)=0$ since $\omega\left(u_{1}, u_{2}, u_{3}\right)=0 \in$ $\Gamma\left(B^{*}\right)$ by (4) in Proposition 5.5. Hence, $U$ with the bracket $\llbracket \cdot, \cdot \rrbracket \mid \Gamma(U) \times \Gamma(U)$ and the anchor $\left.\rho_{Q}\right|_{U}$ is a Lie algebroid.

If $\phi \in \Gamma\left(Q^{*} \wedge Q^{*} \otimes B^{*}\right)$ is the tensor defined as in the proof of Theorem 4.1 by a change of Lagrangian splitting adapted to $D$, then, by Proposition 4.7 in [12],

$$
\llbracket u, u^{\prime} \rrbracket_{1}=\llbracket u, u^{\prime} \rrbracket_{2}+\partial_{B}^{*} \phi\left(u, u^{\prime}\right)
$$

for all $u, u^{\prime}$. But since both splittings $\Sigma^{1}, \Sigma^{2}: B \times_{M} Q \rightarrow \mathbb{E}$ are adapted to $D$, we know that $\sigma_{Q}^{1}(u)$ and $\sigma_{Q}^{2}(u)$ have values in $D$, and their difference $\sigma_{Q}^{1}(u)-$ $\sigma_{Q}^{2}(u)=\widetilde{\phi(u)}$ is a core-linear section of $D \rightarrow B$. Hence it must takes values in $U^{\circ}$, and $\phi\left(u, u^{\prime}\right)$ must so vanish for all $u, u^{\prime} \in \Gamma(U)$. As a consequence, $\llbracket u, u^{\prime} \rrbracket_{1}=$ $\llbracket u, u^{\prime} \rrbracket_{2}$.

The following two corollaries are now easy to prove. The first one was already given in [14].

Corollary 5.7 Let $(\mathcal{M}, \mathcal{Q})$ be a Lie 2-algebroid, and $(\mathbb{E} \rightarrow B, Q \rightarrow M)$ the corresponding VB-Courant algebroid. Then VB-Dirac structures in $\mathbb{E}$ are equivalent to wide Lie 1-subalgebroids of $(\mathcal{M}, \mathcal{Q})$. 
Proof A wide Lie subalgebroid of $(\mathcal{M}, \mathcal{Q})$ is a wide [1]-submanifold $U[-1]$ of $\mathcal{M}$ such that $\mathcal{Q}_{U}\left(\mu^{\star} \xi\right)=\mu^{\star}(\mathcal{Q}(\xi)), \xi \in C^{\infty}(\mathcal{M})$, defines a Lie algebroid structure $\mathcal{Q}_{U}$ on $U$. Here, $\mu: U[-1] \rightarrow \mathcal{M}$ is the submanifold inclusion.

In a splitting $Q[-1] \oplus B^{*}[-2]$ of $\mathcal{M}$, the homological vector field $\mathcal{Q}$ is given by (16)-(18). Choose an open subset $V$ of $M$ with a local frame $\left(u_{1}, \ldots, u_{r}, q_{r+1}, \ldots, q_{l}\right)$ of $Q$ over $V$ such that $\left(u_{1}, \ldots, u_{r}\right)$ is a local frame for $U$ over $V$. Let $\left(\tau_{1}, \ldots, \tau_{l}\right)$ be the dual smooth frame for $Q^{*}$ over $V$. Then we have $\mathcal{Q}_{U}(f)=\mu^{\star}\left(\rho_{Q}^{*} \mathbf{d} f\right)=\rho_{Q}^{*} \mathbf{d} f+U^{\circ}$ for all $f \in C^{\infty}(M)$. This translates easily to $\rho_{U}=\left.\rho_{Q}\right|_{U}$. Then we have $\mathcal{Q}_{U}\left(\tau_{i}+U^{\circ}\right)=\mathcal{Q}_{U}\left(\mu^{\star} \tau_{i}\right)=\mu^{\star}\left(\mathcal{Q}\left(\tau_{k}\right)\right)=$ $-\sum_{i<j}^{r}\left\langle\llbracket u_{i}, u_{j} \rrbracket, \tau_{k}\right\rangle \bar{\tau}_{i} \bar{\tau}_{j}$ for $k=1, \ldots, r$. This shows that the bracket on $U$ must be the restriction to $\Gamma(U)$ of the dull bracket on $\Gamma(Q)$. Finally $0=\mathcal{Q}_{U}\left(\mu^{\star} b\right)=$ $\mu^{\star}(\mathcal{Q}(b))=-\sum_{i<j<k<r} \omega\left(u_{i}, u_{j}, u_{k}\right)(b) \bar{\tau}_{i} \bar{\tau}_{j} \bar{\tau}_{k}$ for all $b \in \Gamma(B)$ shows that $\omega\left(u_{1}, u_{2}, u_{3}\right)$ must be zero for all $u_{1}, u_{2}, u_{3} \in \Gamma(U)$. This is equivalent to (3) in Proposition 5.5 (with $B^{\prime}=B$ ). Note that since $B^{\prime}=B$, (1) and (2) in Proposition 5.5 are trivially satisfied. Hence we can conclude.

The Lie algebroid structure on $U$ is the base Lie algebroid from the VB-algebroid $D \rightarrow B$ in the following corollary. The proof is immediate.

Corollary 5.8 A VB-Dirac structure $(D, B, U, M)$ in a VB-Courant algebroid inherits a linear Lie algebroid structure: $(D \rightarrow B, U \rightarrow M)$ is a VB-algebroid.

\subsection{LA-Dirac Structures}

Assume now that $(\mathbb{E} \rightarrow Q, B \rightarrow M)$ is a metric VB-algebroid, and take a maximal isotropic double subbundle $D$ of $\mathbb{E}$ over the sides $U \subseteq Q$ and $B^{\prime} \subseteq B$. We will study conditions on the self-dual 2-representation defined by a Lagrangian splitting and the linear Lie algebroid structure on $\mathbb{E} \rightarrow Q$, and on $Q$ and on $B^{\prime}$, for $D$ to be an isotropic subalgebroid of $\mathbb{E} \rightarrow Q$ over $U$.

Note the similarity of the following result with Proposition 5.5.

Proposition 5.9 Let $(\mathbb{E}, B, Q, M)$ be a metric VB-algebroid and $\left(D, B^{\prime}, U, M\right) \subseteq \mathbb{E}$ a maximal isotropic double subbundle. Choose a Lagrangian splitting of $\mathbb{E}$ that is adapted to $D$ and consider the corresponding self-dual 2-representation, denoted as usual. Then $D \rightarrow U$ is a subalgebroid of $\mathbb{E} \rightarrow Q$ if and only if

(1) $\partial_{Q}\left(U^{\circ}\right) \subseteq U$,

(2) $\nabla_{b} u \in \Gamma(U)$ for all $u \in \Gamma(U)$ and $b \in \Gamma\left(B^{\prime}\right)$,

(3) $\left[b_{1}, b_{2}\right] \in \Gamma\left(B^{\prime}\right)$ for all $b_{1}, b_{2} \in \Gamma\left(B^{\prime}\right)$,

(4) $R\left(b_{1}, b_{2}\right)$ restricts to a section of $\Gamma\left(\operatorname{Hom}\left(U, U^{\circ}\right)\right)$ for all $b_{1}, b_{2} \in \Gamma\left(B^{\prime}\right)$.

Proof This proof is very similar to the proof of Proposition 5.5, and left to the reader.

Now let $(\mathbb{E}, Q, B, M)$ be an LA-Courant algebroid. A VB-Dirac structure $\left(D, U, B^{\prime}, M\right)$ in $\mathbb{E}$ is an LA-Dirac structure if $\left(D \rightarrow U, B^{\prime} \rightarrow M\right)$ is also a 
subalgebroid of $(\mathbb{E} \rightarrow Q, B \rightarrow M)$. We deduce from Propositions 5.5 and 5.9 a characterisation of LA-Dirac structures.

Proposition 5.10 Let $(\mathbb{E}, B, Q, M)$ be an LA-Courant algebroid and $\left(D, B^{\prime}, U, M\right)$ a maximal isotropic double subbundle of $\mathbb{E}$. Choose a Lagrangian splitting of $\mathbb{E}$ that is adapted to $D$ and consider the corresponding matched self-dual 2-representation and split Lie 2-algebroid. Then $D \rightarrow U$ is an LA-Dirac structure in $\mathbb{E}$ if and only if

(1) $\partial_{B}\left(U^{\circ}\right) \subseteq B^{\prime}$ and $\partial_{Q}\left(U^{\circ}\right) \subseteq U$,

(2) $\nabla_{u} b \in \Gamma\left(B^{\prime}\right)$ for all $u \in \Gamma(U)$ and $b \in \Gamma\left(B^{\prime}\right)$,

(3) $\nabla_{b} u \in \Gamma(U)$ for all $u \in \Gamma(U)$ and $b \in \Gamma\left(B^{\prime}\right)$,

(4) $\llbracket u_{1}, u_{2} \rrbracket \in \Gamma(U)$ for all $u_{1}, u_{2} \in \Gamma(U)$,

(5) $\left[b_{1}, b_{2}\right] \in \Gamma\left(B^{\prime}\right)$ for all $b_{1}, b_{2} \in \Gamma\left(B^{\prime}\right)$,

(6) $\mathbf{i}_{u_{2}} \mathbf{i}_{u_{1}} \omega$ restricts to a section of $\Gamma\left(\operatorname{Hom}\left(B^{\prime}, U^{\circ}\right)\right)$ for all $u_{1}, u_{2} \in \Gamma(U)$,

(7) $R\left(b_{1}, b_{2}\right)$ restricts to a section of $\Gamma\left(\operatorname{Hom}\left(U, U^{\circ}\right)\right)$ for all $b_{1}, b_{2} \in \Gamma\left(B^{\prime}\right)$.

Hence, we also have the following result.

Corollary 5.11 VB-subalgebroids $(D \rightarrow U, B \rightarrow M)$ of a metric VB-algebroid $(\mathbb{E} \rightarrow Q, B \rightarrow M)$ are equivalent to wide coisotropic [1]-submanifolds of the corresponding Poisson [2]-manifold.

LA-Dirac structures $(D \rightarrow U, B \rightarrow M)$ in an LA-Courant algebroid $(\mathbb{E} \rightarrow$ $Q, B \rightarrow M)$ are equivalent to wide coisotropic Lie subalgebroids of the corresponding Poisson Lie 2-algebroid.

Proof Let $U[-1]$ be a [1]-submanifold of a Poisson [2]-manifold $(\mathcal{M},\{\cdot, \cdot\})$. Then $U[-1]$ is coisotropic if and only if $\mu^{\star}(\xi)=\mu^{\star}(\eta)=0$ imply $\mu^{\star}(\{\xi, \eta\})=0$ for all $\xi, \eta \in C^{\infty}(\mathcal{M})$, where $\mu: Q[-1] \rightarrow \mathcal{M}$ is the inclusion. In a local splitting, we find easily that this implies $\partial_{Q}\left(U^{\circ}\right) \subseteq U, \nabla_{b}^{*} \tau \in \Gamma\left(U^{\circ}\right)$ for all $b \in \Gamma(B)$ and $\tau \in \Gamma\left(U^{\circ}\right)$, and the restriction to $U$ of $R\left(b_{1}, b_{2}\right)$ has image in $U^{\circ}$. By Proposition 5.9, we can conclude. The second claim follows with Corollary 5.7.

As a corollary of Theorem 3.7, Propositions 5.5 and 5.9, we get the following theorem.

Theorem 5.12 Let $(\mathbb{E}, B, Q, M)$ be an LA-Courant algebroid and $(D, U, B, M) \subseteq$ $\mathbb{E}$ a (wide) LA-Dirac structure in $\mathbb{E}$.

Then $D$ is a double Lie algebroid with the VB-algebroid structure in Corollary 5.8 and the VB-algebroid structure $(D \rightarrow U, B \rightarrow M)$.

Proof Let us study the two linear Lie algebroid structures on $D$. Choose as before a linear splitting $\Sigma: B \times{ }_{M} Q \rightarrow \mathbb{E}$ that restricts to a linear splitting $\Sigma_{D}: U \times{ }_{M} B \rightarrow D$ of $D$. The LA-Courant algebroid structure of $\mathbb{E}$ is then encoded as in Sections 2.5.2 
and 2.5.1, respectively, by a split Lie 2-algebroid $\left(\partial_{B}: Q^{*} \rightarrow B, \rho_{Q}: Q \rightarrow\right.$ $T M, \llbracket \cdot, \cdot \rrbracket, \nabla, \omega)$ and by a self-dual 2-representation $\left(\nabla, \nabla^{*}, R\right)$ of the Lie algebroid $B$ on $\partial_{Q}=\partial_{Q}^{*}: Q^{*} \rightarrow Q$. By Theorem 3.7, the Dorfman 2-representation and the 2-representation form a matched pair as in Definition 3.1.

By Proposition 5.5 and Corollary 5.6, the restriction to $\Gamma(U)$ of the dull bracket on $\Gamma(Q)$ that is dual to $\Delta$ defines a Lie algebroid structure on $U,\left.\omega\right|_{U \otimes U \otimes Q}$ can be seen as an element of $\Omega^{2}\left(U, \operatorname{Hom}\left(B, U^{\circ}\right)\right)$ and since $\Delta_{u} \tau \in \Gamma\left(U^{\circ}\right)$ for all $u \in$ $\Gamma(U)$ and $\tau \in \Gamma\left(U^{\circ}\right)$, the Dorfman connection $\Delta$ restricts to a map $\Delta^{D}: \Gamma(U) \times$ $\Gamma\left(U^{\circ}\right) \rightarrow \Gamma\left(U^{\circ}\right)$. Since $\Delta_{u}^{D}(f \tau)=f \Delta_{u}^{D} \tau+\rho_{Q}(u)(f) \tau$ and $\Delta_{f u}^{D} \tau=f \Delta_{u}^{D} \tau+$ $\langle u, \tau\rangle \rho_{Q}^{*} \mathbf{d} f=f \Delta_{u} \tau$ for $f \in C^{\infty}(M)$, we find that this restriction is in fact an ordinary connection. Since $\omega\left(u_{1}, u_{2}, u_{3}\right)$ vanishes for all $u_{1}, u_{2}, u_{3} \in \Gamma(U)$, it is then easy to see that the restrictions to sections of $U$ and $U^{\circ}$ of (12), (13) and of (iv) and (v) in the definition of a split Lie 2-algebroid define an ordinary 2-representation. By (32), this 2-representation $\left(\partial_{B}: U^{\circ} \rightarrow B, \nabla, \Delta^{D},\left.\omega\right|_{U \otimes U \otimes Q}\right)$ of the Lie algebroid $U$ on $\partial_{B}: U^{\circ} \rightarrow B$ encodes the VB-algebroid structure that $D \rightarrow B$ inherits from the Courant algebroid $\mathbb{E} \rightarrow B$.

In a similar manner, we find using Proposition 5.9 that the self-dual 2representation $\left(\partial_{Q}: Q^{*} \rightarrow Q, \nabla, \nabla^{*}, R \in \Omega^{2}\left(B, Q^{*} \wedge Q^{*}\right)\right)$ restricts to a 2representation $\left(\partial_{Q}: U^{\circ} \rightarrow U, \nabla^{U}: \Gamma(B) \times \Gamma(U) \rightarrow \Gamma(U), \nabla^{U^{\circ}}: \Gamma(B) \times \Gamma\left(U^{\circ}\right) \rightarrow\right.$ $\left.\Gamma\left(U^{\circ}\right), R \in \Omega^{2}\left(B, \operatorname{Hom}\left(U, U^{\circ}\right)\right)\right)$ of $B$.

A study of the restrictions to sections of $U$ and $U^{\circ}$ of the equations in Definition 3.1 shows then that (M1) restricts to (m2) in Section 2.4 since $\partial_{B}^{*}\left\langle\tau, \nabla^{U} \cdot u\right\rangle=0$ for all $u \in \Gamma(U)$ and $\tau \in \Gamma\left(U^{\circ}\right)$. The equations (M2), and (M3) immediately yield (m3) and (m6), respectively. (M4) restricts to (m5) since $\left\langle R(\cdot, b) u_{1}, u_{2}\right\rangle=0$ for all $u_{1}, u_{2} \in \Gamma(U)$ and $b \in \Gamma(B)$. (M5) restricts to (m7) since the right-hand side of (M5) in (1) of Remark 3.2 vanishes. Finally, (21) restricts to (m1) and (22) restricts to (m4) since $\left\langle\nabla_{\nabla . b} u, \tau\right\rangle=0$ for all $b \in \Gamma(B), u \in \Gamma(U)$ and $\tau \in \Gamma\left(U^{\circ}\right)$. Thus, the two 2-representations describing the sides of $D$ given the splitting $\Sigma_{D}$ form a matched pair, which implies that $D$ is a double Lie algebroid (see [5] or Section 2.4 for a quick summary of this paper).

Note finally that with a different approach as the one adopted in this paper, we could deduce the main result in [5] from our Theorem 3.7. Once one has 'directly' proved that for each double Lie algebroid $(D, A, B, M)$ with core $C$, the direct sum over $B$ of $D$ and $D_{B}^{*}$ defines an LA-Courant algebroid $\left(D \oplus_{B}\left(D_{B}^{*}\right), A \oplus C^{*}, B, M\right)$ as in Section 3.3.3, then one can use the last theorem to deduce the equations in Section 2.4 from the ones in Definition 3.1 and in Remark 3.2: by construction, the double vector subbundle $D$ of $D \oplus_{B}\left(D_{B}^{*}\right)$ is a VB-Dirac structure in $D \oplus_{B}\left(D_{B}^{*}\right) \rightarrow B$ and a linear Lie subalgebroid in $D \oplus_{B}\left(D_{B}^{*}\right) \rightarrow A \oplus C^{*}$. Instead, we have chosen to use the main theorem in [5] to prove that $\left(D \oplus_{B}\right.$ $\left.\left(D_{B}^{*}\right), A \oplus C^{*}, B, M\right)$ is an LA-Courant algebroid, see Section 3.3.3. By the complexity of Li-Bland's definition of an LA-Courant algebroid, this is the most simple approach. 


\subsection{Pseudo-Dirac Structures}

We explain here the notion of pseudo-Dirac structures that was introduced in [14, 15] and we compare it with our approach to VB- and LA-Dirac structures in the tangent of a Courant algebroid. Consider a VB-Courant algebroid $\mathbb{E}$ with core $Q^{*}$, and a double vector subbundle in $\mathbb{E}$ with core $K$, as in the following diagrams.
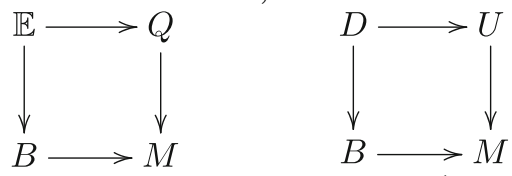

Consider the restriction $\left.\mathbb{E}\right|_{U}$ of $\mathbb{E}$ to $U$; i.e. $\left.\mathbb{E}\right|_{U}=\pi_{Q}^{-1}(U)$. This is a double vector bundle with sides $B$ and $U$ and with core $Q^{*}$. The total quotient of $\left.\mathbb{E}\right|_{U}$ by $D$ is the map q from
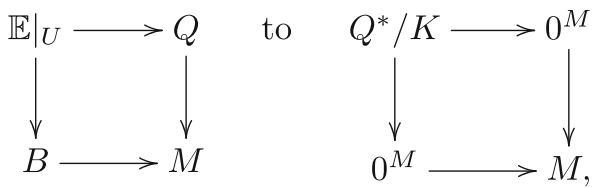

defined by

$$
\mathrm{q}(e)=\bar{\tau} \Leftrightarrow e-\tau^{\dagger} \in D .
$$

After the choice of a linear splitting of $\mathbb{E}$ that is adapted to $D$, we know that each element of $\left.\mathbb{E}\right|_{U}$ can be written $\sigma_{Q}(u)\left(b_{m}\right)+\tau^{\dagger}\left(b_{m}\right)$ for some $u \in \Gamma(U)$, $\tau \in \Gamma\left(Q^{*}\right)$ and $b_{m} \in B$. The image of $\sigma_{Q}(u)\left(b_{m}\right)+\tau^{\dagger}\left(b_{m}\right)$ under $\mathrm{q}$ is then simply $\bar{\tau}(m)$. Conversely it is easy to see that $D$ can be recovered from $q$. Recall that if $e_{1}=\sigma_{Q}\left(u_{1}\right)\left(b_{m}\right)+\tau_{1}^{\dagger}\left(b_{m}\right)$ and $e_{2}=\sigma_{Q}\left(u_{2}\right)\left(b_{m}\right)+\tau_{2}^{\dagger}\left(b_{m}\right) \in \mathbb{E}$, then

$$
\begin{aligned}
\left\langle e_{1}, e_{2}\right\rangle & =\left\langle\sigma_{Q}\left(u_{1}\right)\left(b_{m}\right)+\tau_{1}^{\dagger}\left(b_{m}\right), \sigma_{Q}\left(u_{2}\right)\left(b_{m}\right)+\tau_{2}^{\dagger}\left(b_{m}\right)\right\rangle \\
& =\ell_{\Lambda\left(u_{1}, u_{2}\right)}\left(b_{m}\right)+\left\langle u_{1}(m), \tau_{2}(m)\right\rangle+\left\langle u_{2}(m), \tau_{1}(m)\right\rangle .
\end{aligned}
$$

In particular, $\left\langle e_{1}, e_{2}\right\rangle=\left\langle\pi_{Q}\left(e_{1}\right), \mathrm{q}\left(e_{2}\right)\right\rangle+\left\langle\pi_{Q}\left(e_{2}\right), \mathrm{q}\left(e_{1}\right)\right\rangle$ for all $e_{1},\left.e_{2} \in \mathbb{E}\right|_{U}$ if and only if $\left.\Lambda\right|_{U \otimes U}$ vanishes and $K=U^{\circ}$, i.e. if and only if $D$ is maximal isotropic (Proposition 5.2).

Now we recall Li-Bland's definition of a pseudo-Dirac structure [15].

Definition 5.13 Let $\mathrm{E} \rightarrow M$ be a Courant algebroid. A pseudo-Dirac structure is a pair $\left(U, \nabla^{p}\right)$ consisting of a subbundle $U \subseteq \mathrm{E}$ together with a map $\nabla^{p}: \Gamma(U) \rightarrow$ $\Omega^{1}\left(M, U^{*}\right)$ satisfying

(1) $\nabla^{p}(f u)=f \nabla^{p} u+\mathbf{d} f \otimes\langle u, \cdot\rangle$,

(2) $\mathbf{d}\left\langle u_{1}, u_{2}\right\rangle=\left\langle\nabla^{p} u_{1}, u_{2}\right\rangle+\left\langle u_{1}, \nabla^{p} u_{2}\right\rangle$,

(3) $\llbracket u_{1}, u_{2} \rrbracket_{p}:=\llbracket u_{1}, u_{2} \rrbracket_{\mathrm{E}}-\rho^{*}\left\langle\nabla^{p} u_{1}, u_{2}\right\rangle$ defines a bracket $\Gamma(U) \times \Gamma(U) \rightarrow$ $\Gamma(U)$,

(4) and

$$
\begin{aligned}
& \left(\left\langle\llbracket u_{1}, u_{2} \rrbracket_{p}, \nabla^{p} u_{3}\right\rangle+\mathbf{i}_{\rho\left(u_{1}\right)} \mathbf{d}\left\langle\nabla^{p} u_{2}, u_{3}\right\rangle\right)+\text { c.p. } \\
& \quad+\mathbf{d}\left(\left\langle\nabla_{\rho\left(u_{1}\right)}^{p} u_{2}-\nabla_{\rho\left(u_{2}\right)}^{p} u_{1}, u_{3}\right\rangle-\left\langle\llbracket u_{1}, u_{2} \rrbracket_{p}, u_{3}\right\rangle\right)=0
\end{aligned}
$$


for all $u_{1}, u_{2}, u_{3} \in \Gamma(U)$ and $f \in C^{\infty}(M)$.

Consider the tangent double ( $T \mathrm{E}, T M$, E, $M$ ) where $\mathrm{E}$ is a Courant algebroid over $M$. Choose a linear (wide) Dirac structure $D$ in $T \mathrm{E}$, over the side $U \subseteq \mathrm{E}$ and a metric connection $\nabla: \mathfrak{X}(M) \times \Gamma(\mathrm{E}) \rightarrow \Gamma(\mathrm{E})$ that is adapted to $D$. Li-Bland defines the pseudo-Dirac structure associated to $D[15]$ as the map $\nabla^{p}: \Gamma(U) \rightarrow \Omega^{1}\left(M, U^{*}\right)$ that is defined by $\nabla^{p} u=\mathrm{q} \circ T u$ for all $u \in \Gamma(U)$. By definition of $\sigma_{\mathrm{E}}^{\nabla}$, we have $T u=\sigma_{\mathrm{E}}^{\nabla}(u)+\widetilde{\nabla \cdot u}$ and we find that $\nabla^{p} u\left(v_{m}\right)=\overline{\nabla_{v_{m}} u}=[\nabla]_{v_{m}} u$. The pseudoDirac structure is nothing else than the invariant part of the metric connection that is adapted to $D$ (Remark 5.4). Condition (2) in Definition 5.13 is then

$$
\mathbf{d}\left\langle u_{1}, u_{2}\right\rangle=\left\langle T u_{1}, T u_{2}\right\rangle_{T \mathrm{E}}=\left\langle u_{1}, \nabla^{p} u_{2}\right\rangle+\left\langle u_{2}, \nabla^{p} u_{1}\right\rangle
$$

for all $u_{1}, u_{2} \in \Gamma(U)$ and Condition (1) is

$$
\nabla^{p}(\varphi \cdot u)=\overline{\nabla \cdot(\varphi \cdot u)}=\varphi \cdot \overline{\nabla \cdot u}+\mathbf{d} \varphi \otimes \bar{u}=\varphi \cdot \nabla^{p} u+\mathbf{d} \varphi \otimes \bar{u} .
$$

The bracket $\llbracket \cdot \cdot, \cdot \rrbracket_{p}$ is then

$$
\llbracket u_{1}, u_{2} \rrbracket_{p}=\llbracket u_{1}, u_{2} \rrbracket_{\mathrm{E}}-\rho^{*}\left\langle\nabla^{p} u_{1}, u_{2}\right\rangle=\llbracket u_{1}, u_{2} \rrbracket_{\mathrm{E}}-\rho^{*}\left\langle\nabla \cdot u_{1}, u_{2}\right\rangle=\llbracket u_{1}, u_{2} \rrbracket_{\nabla},
$$

the bracket defined in (14). Finally, a straightforward computation shows that the lefthand side of (33) equals $R_{\Delta}^{\text {bas }}\left(u_{1}, u_{2}\right)^{*} u_{3} \in \Gamma\left(B^{*}\right)$, which is zero by Proposition 5.5. Li-Bland proves that the bracket $\llbracket \cdot, \cdot \rrbracket_{p}$ defines a Lie algebroid structure on $U$. More explicitly, he finds that the left-hand side $\Psi\left(u_{1}, u_{2}, u_{3}\right)$ of (33) defines a tensor $\Psi \in$ $\Omega^{3}\left(U, T^{*} M\right)$ that is related as follows to the Jacobiator of $\llbracket \cdot, \cdot \rrbracket_{p}: \mathrm{Jac}_{\llbracket \cdot, \cdot \rrbracket_{p}}=\left(\boldsymbol{\beta}^{-1}\right.$ 。 $\left.\rho_{\mathrm{E}}^{*}\right) \Psi$. He proves so that (wide) linear Dirac structures in $T \mathrm{E}$ are in bijection with pseudo-Dirac structures on E. Hence, our result in Proposition 5.5 is a generalisation of Li-Bland's result to linear Dirac structures in general VB-Courant algebroids.

Further, our Theorem 5.9 can be formulated as follows in Li-Bland's setting.

Theorem 5.14 In the correspondence of linear Dirac structures with pseudo-Dirac connections in [15], LA-Dirac structures correspond to pseudo-Dirac connections $\left(U, \nabla^{p}\right)$ such that

(1) $U \subseteq \mathrm{E}$ is an isotropic (or 'quadratic') subbundle, i.e. $U^{\perp} \subseteq U$,

(2) $\nabla^{p}$ sends $U^{\perp}$ to zero and so, by Condition (2) in Definition 5.13, has image in $U / U^{\perp} \subseteq \mathrm{E} / U^{\perp} \simeq U^{*}$,

(3) the induced ordinary connection $\overline{\nabla^{p}}: \Gamma\left(U / U^{\perp}\right) \rightarrow \Omega^{1}\left(M, U / U^{\perp}\right)$ is flat.

We propose to call these pseudo-Dirac connections quadratic pseudo-Dirac connections. Note that $\overline{\nabla^{p}}$ equals $\bar{\nabla}: \mathfrak{X}(M) \times \Gamma\left(U / U^{\perp}\right) \rightarrow \Gamma\left(U / U^{\perp}\right) \bar{\nabla}_{X} \bar{u}=\bar{\nabla}_{X} u$, $u \in \Gamma(U)$ and $X \in \mathfrak{X}(M)$, for any metric connection $\nabla: \mathfrak{X}(M) \times \Gamma(U) \rightarrow \Gamma(U)$ such that $[\nabla]=\nabla^{p}$. Such a connection must preserve $U$ by Condition (2) in Proposition 5.9, and so also $U^{\perp}$ since it is metric. The condition $R_{\nabla}\left(X_{1}, X_{2}\right) u \in \Gamma\left(U^{\perp}\right)$ for all $X_{1}, X_{2} \in \mathfrak{X}(M)$ and $u \in \Gamma(U)$ in Proposition 5.9 is then equivalent to $R_{\bar{\nabla}}=0$. 


\subsection{The Manin Pair Associated to an LA-Dirac Structure}

Consider as before an LA-Courant algebroid $\mathbb{E}$ with sides $B$ and $Q$ and with core $Q^{*}$, and an LA-Dirac structure $D$
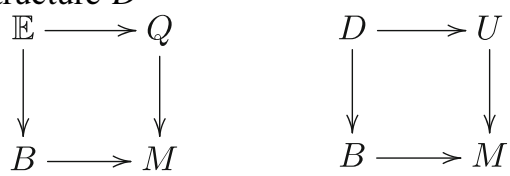

in $\mathbb{E}$ with core $U^{\circ}$. Since $\partial_{Q}$ restricts to a map from $U^{\circ}$ to $U$, we can define the vector bundle

$$
\mathbb{B}=\frac{U \oplus Q^{*}}{\operatorname{graph}\left(-\left.\partial_{Q}\right|_{U^{\circ}}\right)} \rightarrow M .
$$

This vector bundle is anchored by the map

$$
\rho_{\mathbb{B}}: \mathbb{B} \rightarrow T M, \quad \rho_{\mathbb{B}}(u \oplus \tau)=\rho_{Q}\left(u+\partial_{Q} \tau\right)=\rho_{Q}(u)+\rho_{B}\left(\partial_{B} \tau\right) .
$$

Note that this map is well-defined because

$$
\rho_{\mathbb{B}}\left(-\partial_{Q} \tau \oplus \tau\right)=\rho_{Q}\left(-\partial_{Q} \tau+\partial_{Q} \tau\right)=0
$$

for all $\tau \in U^{\circ}$. We will show that there is a symmetric non-degenerate pairing $\langle\cdot, \cdot\rangle_{\mathbb{B}}$ on $\mathbb{B} \times{ }_{M} \mathbb{B}$ and a bracket $\llbracket \cdot, \cdot \rrbracket_{\mathbb{B}}$ on $\Gamma(\mathbb{B})$ such that

$$
\left(\mathbb{B} \rightarrow M, \rho_{\mathbb{B}},\langle\cdot, \cdot\rangle_{\mathbb{B}}, \llbracket \cdot, \cdot \rrbracket_{\mathbb{B}}\right)
$$

is a Courant-algebroid. We define the pairing on $\mathbb{B}$ by

$$
\left\langle u_{1} \oplus \tau_{1}, u_{2} \oplus \tau_{2}\right\rangle_{\mathbb{B}}=\left\langle u_{1}, \tau_{1}\right\rangle+\left\langle u_{2}, \tau_{2}\right\rangle+\left\langle\tau_{1}, \partial_{Q} \tau_{2}\right\rangle .
$$

It is easy to check that this pairing is well-defined and non-degenerate and that the induced map $\mathcal{D}_{\mathbb{B}}: C^{\infty}(M) \rightarrow \Gamma(\mathbb{B})$ given by

$$
\left\langle\mathcal{D}_{\mathbb{B}} f, u \oplus \tau\right\rangle_{\mathbb{F}}=\rho_{\mathbb{B}}(u \oplus \tau)(f)
$$

can alternatively be defined by $\mathcal{D}_{\mathbb{B}} f=0 \oplus \rho_{Q}^{*} \mathbf{d} f$.

Choose as before a Lagrangian splitting of $\mathbb{E}$ that is adapted to $D$, and recall that the linear Courant algebroid structure and the linear Lie algebroid structure on $\mathbb{E}$ are then encoded by a split Lie 2-algebroid and by a self-dual 2-representation, respectively, both denoted as usual. We define the bracket on $\Gamma(\mathbb{B})$ by

$$
\begin{aligned}
\llbracket u_{1} \oplus \tau_{1}, u_{2} \oplus \tau_{2} \rrbracket_{\mathbb{B}}= & \left(\llbracket u_{1}, u_{2} \rrbracket_{U}+\nabla_{\partial_{B}} \tau_{1} u_{2}-\nabla_{\partial_{B}} \tau_{2} u_{1}\right) \oplus\left(\llbracket \tau_{1}, \tau_{2} \rrbracket_{Q^{*}}\right. \\
& \left.+\Delta_{u_{1}} \tau_{2}-\Delta_{u_{2}} \tau_{1}+\rho_{Q}^{*} \mathbf{d}\left\langle\tau_{1}, u_{2}\right\rangle\right) .
\end{aligned}
$$

A quick computation as the one at the end of the proof of Theorem 4.1 show that this bracket does not depend on the choice of Lagrangian splitting.

Theorem 5.15 Let $(D, U, B, M)$ be an LA-Dirac structure in a LA-Courant algebroid $(\mathbb{E}, Q, B, M)$. Then the vector bundle

$$
\mathbb{B}=\frac{U \oplus Q^{*}}{\operatorname{graph}\left(-\left.\partial_{Q}\right|_{U^{\circ}}\right)} \rightarrow M,
$$

with the anchor $\rho_{\mathbb{B}}$, the pairing $\langle\cdot, \cdot\rangle_{\mathbb{B}}$ and the bracket $\llbracket \cdot, \cdot \rrbracket_{\mathbb{B}}$, is a Courant algebroid. Further, $U$ is a Dirac structure in $\mathbb{B}$, via the inclusion $U \hookrightarrow \mathbb{B}, u \mapsto u \oplus 0$. 
The proof of Theorem 5.15 can be found in Appendix A.

Corollary 5.16 Let $(D, U, B, M)$ be an LA-Dirac structure in an LA-Courant algebroid $(\mathbb{E}, B, Q, M)$ (with core $\left.Q^{*}\right)$. The Manin pair $(\mathbb{B}, U)$ defined in Theorem 5.15 and the degenerate Courant algebroid $Q^{*}$ satisfy the following conditions:

(1) There is a morphism $\psi: Q^{*} \rightarrow \mathbb{B}$ of degenerate Courant algebroids and an embedding $\iota: U \rightarrow Q$ over the identity on $M$

(2) $\iota$ is compatible with the anchors: $\rho_{Q} \circ \iota=\left.\rho_{\mathbb{B}}\right|_{U}$,

(3) $\psi\left(Q^{*}\right)+U=\mathbb{B}$ and

(4) $\langle\psi(\tau), u\rangle_{\mathbb{B}}=\langle\iota(u), \tau\rangle$ for all $\tau \in Q^{*}$ and $u \in U$.

Proof Take an LA-Dirac structure $(D, U, B, M)$ in an LA-Courant algebroid $(\mathbb{E}, Q, B, M)$. The morphism $\psi: Q^{*} \rightarrow \mathbb{B}$ defined by $\psi(\tau) \mapsto 0 \oplus \tau$ is obviously a morphism of degenerate Courant algebroids. Conditions (1)-(4) are then immediate.

Conversely take a Manin pair $(\mathbb{B}, U)$ over $M$ satisfying with $Q^{*}$ the conditions in Corollary 5.16 and identify $U$ with a subbundle of $Q$. If $\tau \in U^{\circ} \subseteq Q^{*}$, then $\psi(\tau)$ satisfies

$$
\langle u, \psi(\tau)\rangle_{\mathbb{B}}=\langle u, \tau\rangle=0
$$

for all $u \in U$. Since $U$ is a Dirac structure, we find that $\psi$ restricts to a map $U^{\circ} \rightarrow U$. Conversely, we find easily that $\psi(\tau) \in U$ if and only if $\tau \in U^{\circ}$. Next choose $\tau_{1} \in U^{\circ}$ and $\tau_{2} \in Q^{*}$. Then since $\psi\left(\tau_{1}\right) \in U$,

$$
\left\langle\psi\left(\tau_{1}\right), \tau_{2}\right\rangle=\left\langle\psi\left(\tau_{1}\right), \psi\left(\tau_{2}\right)\right\rangle_{\mathbb{B}}=\left\langle\tau_{1}, \tau_{2}\right\rangle_{Q^{*}}=\left\langle\partial_{Q} \tau_{1}, \tau_{2}\right\rangle,
$$

which shows that $\left.\psi\right|_{U^{\circ}}=\left.\partial_{Q}\right|_{U^{\circ}}$. In particular, $\partial_{Q}$ sends $U^{\circ}$ to $U$, and $U^{\circ}$ is isotropic in $Q^{*}$. Consider the vector bundle map $U \oplus Q^{*} \rightarrow \mathbb{B},(u, \tau) \mapsto u+\psi(\tau)$. By assumption, this map is surjective. Its kernel is the set of pairs $(u, \tau)$ with $u=-\psi(\tau)$, i.e. the graph of $-\left.\partial_{Q}\right|_{U^{\circ}}: U^{\circ} \rightarrow U$. It follows that

$$
\mathbb{B} \simeq \frac{U \oplus Q^{*}}{\operatorname{graph}\left(-\left.\partial_{Q}\right|_{U^{\circ}}: U^{\circ} \rightarrow U\right)} .
$$

Hence, we can use the notation $u \oplus \tau$ for $\overline{u+\psi(\tau)} \in \mathbb{B}$.

In the case of an LA-Courant algebroid ( $\left.T A \oplus_{A} T^{*} A, T M \oplus A^{*}, A, M\right)$ as in Section 3.3.2, for a Lie algebroid $A$, we could show in [11] that Manin pairs as in Corollary 5.16 are in bijection with LA-Dirac structures on $A$. That is, given a Manin pair $(\mathbb{B}, U)$ with an inclusion $U \hookrightarrow T M \oplus A^{*}$ and a degenerate Courant algebroid morphism $A \oplus T^{*} M \rightarrow \mathbb{B}$ satisfying (1)-(4), then via (37), there exists a Lagrangian splitting of $T A \oplus_{A} T^{*} A$ such that the Courant bracket on $\mathbb{B}$ is given by (36).

\section{Compliance with Ethical Standards}

Conflict of interests The author states that there is no conflict of interest.

Funding Information Open Access funding provided by Projekt DEAL. 
Open Access This article is licensed under a Creative Commons Attribution 4.0 International License, which permits use, sharing, adaptation, distribution and reproduction in any medium or format, as long as you give appropriate credit to the original author(s) and the source, provide a link to the Creative Commons licence, and indicate if changes were made. The images or other third party material in this article are included in the article's Creative Commons licence, unless indicated otherwise in a credit line to the material. If material is not included in the article's Creative Commons licence and your intended use is not permitted by statutory regulation or exceeds the permitted use, you will need to obtain permission directly from the copyright holder. To view a copy of this licence, visit http://creativecommonshorg/licenses/by/4. $0 \%$

\section{Appendix A: Proof of Theorem 5.15}

Note that in the following computations, we will make use of the identity $\partial_{Q}=\partial_{Q}^{*}$ without always mentioning it. We begin by proving the following two lemmas.

Lemma A.1 Consider an LA-Courant algebroid $(\mathbb{E}, Q, B, M)$. The bracket $\llbracket \cdot, \cdot \rrbracket_{Q *}$ on sections of the core $Q^{*}$ satisfies the following equation:

$$
\begin{aligned}
R\left(\partial_{B} \tau_{1}, \partial_{B} \tau_{2}\right) q= & -\Delta_{q} \llbracket \tau_{1}, \tau_{2} \rrbracket Q^{*}+\llbracket \Delta_{q} \tau_{1}, \tau_{2} \rrbracket Q^{*}+\llbracket \tau_{1}, \Delta_{q} \tau_{2} \rrbracket Q^{*} \\
& +\Delta_{\nabla_{\partial_{B} \tau_{2}} q} \tau_{1}-\Delta_{\nabla_{\partial_{B} \tau_{1}} q} \tau_{2}-\rho_{Q}^{*} \mathbf{d}\left\langle\tau_{1}, \nabla_{\partial_{B} \tau_{2}} q\right\rangle
\end{aligned}
$$

for all $q \in \Gamma(Q)$ and $\tau_{1}, \tau_{2} \in \Gamma\left(Q^{*}\right)$.

Proof The proof is just a computation using (M1) and (22). We have

$$
\begin{aligned}
& \Delta_{q} \llbracket \tau_{1}, \tau_{2} \rrbracket Q^{*}-\llbracket \Delta_{q} \tau_{1}, \tau_{2} \rrbracket Q^{*}-\llbracket \tau_{1}, \Delta_{q} \tau_{2} \rrbracket Q^{*}+\Delta_{\nabla_{\partial_{B}} \tau_{1} q} \tau_{2} \\
& -\Delta_{\nabla_{\partial_{B} \tau_{2}} q} \tau_{1}+\rho_{Q}^{*} \mathbf{d}\left\langle\tau_{1}, \nabla_{\partial_{B} \tau_{2}} q\right\rangle \\
= & \Delta_{q} \Delta_{\partial_{Q} \tau_{1}} \tau_{2}-\Delta_{q} \nabla_{\partial_{B} \tau_{2}}^{*} \tau_{1}-\Delta_{\partial_{Q}\left(\Delta_{q} \tau_{1}\right)} \tau_{2}+\nabla_{\partial_{B} \tau_{2}}^{*} \Delta_{q} \tau_{1}-\Delta_{\partial_{Q} \tau_{1}} \Delta_{q} \tau_{2} \\
& +\nabla_{\partial_{B}\left(\Delta_{q} \tau_{2}\right)}^{*} \tau_{1}+\Delta_{\nabla_{\partial_{B} \tau_{1}}} \tau_{2}-\Delta_{\nabla_{\partial_{B} \tau_{2}} q} \tau_{1}+\rho_{Q}^{*} \mathbf{d}\left\langle\tau_{1}, \nabla_{\partial_{B} \tau_{2}} q\right\rangle
\end{aligned}
$$

Replacing $\Delta_{q} \Delta_{\partial_{Q} \tau_{1}} \tau_{2}-\Delta_{\partial_{Q} \tau_{1}} \Delta_{q} \tau_{2}$ by $R_{\Delta}\left(q, \partial_{Q} \tau_{1}\right) \tau_{2}+\Delta_{\llbracket q, \partial_{Q} \tau_{1} \rrbracket} \tau_{2}$ and reordering the terms yields

$$
\begin{aligned}
& R_{\Delta}\left(q, \partial_{Q} \tau_{1}\right) \tau_{2}+\Delta_{\llbracket q, \partial_{Q} \tau_{1} \rrbracket-\partial_{Q}\left(\Delta_{q} \tau_{1}\right)+\nabla_{\partial_{B} \tau_{1}} q} \tau_{2}-\Delta_{q} \nabla_{\partial_{B} \tau_{2}}^{*} \tau_{1}+\nabla_{\partial_{B} \tau_{2}}^{*} \Delta_{q} \tau_{1} \\
& +\nabla_{\partial_{B}\left(\Delta_{q} \tau_{2}\right)}^{*} \tau_{1}-\Delta_{\nabla_{\partial_{B} \tau_{2}} q} \tau_{1}+\rho_{Q}^{*} \mathbf{d}\left\langle\tau_{1}, \nabla_{\partial_{B} \tau_{2}} q\right\rangle .
\end{aligned}
$$

Since $R_{\Delta}\left(q, \partial_{Q} \tau_{1}\right) \tau_{2}=\left\langle\mathbf{i}_{\partial_{Q} \tau_{1}} \mathbf{i}_{q} \omega, \partial_{B} \tau_{2}\right\rangle$ by (13), we can now use (22) and $\nabla_{q} \circ \partial_{B}=$ $\partial_{B} \circ \Delta_{q}$ to replace

$$
R_{\Delta}\left(q, \partial_{Q} \tau_{1}\right) \tau_{2}-\Delta_{q} \nabla_{\partial_{B} \tau_{2}}^{*} \tau_{1}+\nabla_{\partial_{B} \tau_{2}}^{*} \Delta_{q} \tau_{1}-\Delta_{\nabla_{\partial_{B} \tau_{2}}} \tau_{1}+\nabla_{\nabla_{q} \partial_{B} \tau_{2}}^{*} \tau_{1}
$$

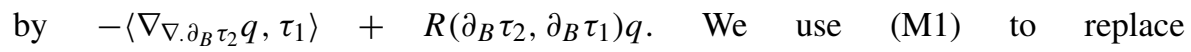
$\Delta_{\llbracket q, \partial_{Q} \tau_{1} \rrbracket-\partial_{Q}\left(\Delta_{q} \tau_{1}\right)+\nabla_{\partial_{B} \tau_{1}} q} \tau_{2}$ by $-\Delta_{\partial_{B}^{*}\left\langle\tau_{1}, \nabla . q\right\rangle} \tau_{2}$. These two steps yield that the right hand side of our equation is

$$
-\left\langle\nabla_{\nabla . \partial_{B} \tau_{2}} q, \tau_{1}\right\rangle+R\left(\partial_{B} \tau_{2}, \partial_{B} \tau_{1}\right) q-\Delta_{\partial_{B}^{*}\left\langle\tau_{1}, \nabla \cdot q\right\rangle} \tau_{2}+\rho_{Q}^{*} \mathbf{d}\left\langle\tau_{1}, \nabla_{\partial_{B} \tau_{2}} q\right\rangle .
$$

To conclude, let us show that

$$
-\left\langle\nabla_{\nabla . \partial_{B} \tau_{2}} q, \tau_{1}\right\rangle-\Delta_{\partial_{B}^{*}\left\langle\tau_{1}, \nabla . q\right\rangle} \tau_{2}+\rho_{Q}^{*} \mathbf{d}\left\langle\tau_{1}, \nabla_{\partial_{B} \tau_{2}} q\right\rangle \in \Gamma\left(Q^{*}\right)
$$


vanishes. On $q^{\prime} \in \Gamma(Q)$, this is

$$
\begin{aligned}
& -\left\langle\nabla_{\nabla_{q^{\prime}}\left(\partial_{B} \tau_{2}\right)} q, \tau_{1}\right\rangle+\left\langle\llbracket \partial_{B}^{*}\left\langle\nabla . q, \tau_{1}\right\rangle, q^{\prime} \rrbracket, \tau_{2}\right\rangle+\rho_{Q}\left(q^{\prime}\right)\left\langle\tau_{1}, \nabla_{\partial_{B} \tau_{2}} q\right\rangle \\
= & -\left\langle\nabla_{\nabla_{q^{\prime}}\left(\partial_{B} \tau_{2}\right)} q, \tau_{1}\right\rangle+\left\langle\Delta_{q^{\prime}} \tau_{2}, \partial_{B}^{*}\left(\left\langle\nabla . q, \tau_{1}\right\rangle\right)\right\rangle \\
= & -\left\langle\nabla_{\nabla_{q^{\prime}}\left(\partial_{B} \tau_{2}\right)} q, \tau_{1}\right\rangle+\left\langle\nabla_{\partial_{B}\left(\Delta_{q^{\prime}} \tau_{2}\right)} q, \tau_{1}\right\rangle=0 .
\end{aligned}
$$

We have used (11) and (2) in the first line, as well as for the first equality. To conclude, we have used $\partial_{B} \circ \Delta_{q^{\prime}}=\nabla_{q^{\prime}} \circ \partial_{B}$ by (12).

Lemma A.2 The bracket on $Q^{*}$ satisfies

$$
\llbracket \rho_{Q}^{*} \mathbf{d} f, \tau \rrbracket Q^{*}=0
$$

for all $f \in C^{\infty}(M)$ and $\tau \in \Gamma\left(Q^{*}\right)$.

Proof By (21), we have $\llbracket \rho_{Q}^{*} \mathbf{d} f, \tau \rrbracket_{Q^{*}}=\nabla_{\partial_{B} \rho_{Q}^{*} \mathbf{d} f} \tau-\Delta_{\partial_{Q} \tau}\left(\rho_{Q}^{*} \mathbf{d} f\right)+$ $\rho_{Q}^{*} \mathbf{d}\left(\left(\rho_{Q} \partial_{Q} \tau\right) f\right)$. But $\partial_{B} \rho_{Q}^{*}=0$ by (11) and $\Delta_{\partial_{Q} \tau}\left(\rho_{Q}^{*} \mathbf{d} f\right)=\rho_{Q}^{*} \mathbf{d}\left(\rho_{Q}\left(\partial_{Q} \tau\right)(f)\right)$ by (3).

Now we check that the bracket $\llbracket \cdot, \cdot \rrbracket_{\mathbb{B}}$ in Theorem 5.15 is well-defined. We have for all $v \in \Gamma\left(U^{\circ}\right), \tau \in \Gamma\left(Q^{*}\right)$ and $u \in \Gamma(U)$ :

$$
\begin{aligned}
\llbracket u \oplus \tau,\left(-\partial_{Q} v\right) \oplus v \rrbracket=\left(-\llbracket u, \partial_{Q} v \rrbracket_{U}+\nabla_{\partial_{B} \tau}\left(-\partial_{Q} v\right)-\nabla_{\partial_{B} v} u\right) \\
\oplus\left(\llbracket \tau, v \rrbracket_{Q^{*}}+\Delta_{u} v-\Delta_{-\partial_{Q} v} \tau+\rho_{Q}^{*} \mathbf{d}\left\langle\tau,-\partial_{Q} v\right\rangle\right) .
\end{aligned}
$$

By (M1), the properties of 2-representations and (21), this is

$$
\begin{aligned}
& \left(-\partial_{Q}\left(\Delta_{u} v\right)+\partial_{B}^{*}\langle v, \nabla . u\rangle+\nabla_{\partial_{B} v u}-\partial_{Q} \nabla_{\partial_{B} \tau}^{*} v-\nabla_{\partial_{B} v} u\right) \\
& \oplus\left(-\Delta_{\partial Q} v+\nabla_{\partial_{B} \tau} v+\rho_{Q}^{*} \mathbf{d}\langle\tau, \partial \widehat{Q} v\rangle+\Delta_{u} v+\Delta_{\partial Q} \tau-\rho_{Q}^{*} \mathbf{d}\left\langle\tau, \partial_{Q} v\right\rangle\right) \\
= & \left(-\partial_{Q}\left(\Delta_{u} v\right)+\partial_{B}^{*}\langle v, \nabla \cdot u\rangle-\partial_{Q} \nabla_{\partial_{B} \tau}^{*} v\right) \oplus\left(\nabla_{\partial_{B} \tau}^{*} v+\Delta_{u} v\right) .
\end{aligned}
$$

Since $v \in \Gamma\left(U^{\circ}\right)$ and $\nabla_{b}$ preserves $\Gamma(U)$ for all $b \in \Gamma(B)$, the section $\langle v, \nabla . u\rangle$ of $B^{*}$ vanishes and we get

$$
\llbracket u \oplus \tau,\left(-\partial_{Q} v\right) \oplus v \rrbracket=\left(-\partial_{Q}\left(\Delta_{u} v+\nabla_{\partial_{B} \tau}^{*} v\right)\right) \oplus\left(\nabla_{\partial_{B} \tau}^{*} v+\Delta_{u} v\right) .
$$

Because $\Delta_{u}$ preserves as well $\Gamma\left(U^{\circ}\right)$, the sum $\nabla_{\partial_{B} \tau}^{*} v+\Delta_{u} v$ is a section of $U^{\circ}$, and so $\llbracket u \oplus \tau,\left(-\partial_{Q} v\right) \oplus v \rrbracket$ is zero in $\mathbb{B}$.

We now check the Courant algebroid axioms (CA1), (CA2) and (CA3). The last one, (CA3), is immediate:

$$
\begin{aligned}
& \llbracket u_{1} \oplus \tau_{1}, u_{2} \oplus \tau_{2} \rrbracket_{\mathbb{B}}+\llbracket u_{2} \oplus \tau_{2}, u_{1} \oplus \tau_{1} \rrbracket_{\mathbb{B}} \\
= & 0 \oplus\left(\rho_{Q}^{*} \mathbf{d}\left\langle\tau_{1}, \partial \tau_{2}\right\rangle+\rho_{Q}^{*} \mathbf{d}\left\langle\tau_{1}, u_{2}\right\rangle+\rho_{Q}^{*} \mathbf{d}\left\langle\tau_{2}, u_{1}\right\rangle\right) \\
= & 0 \oplus \rho_{Q}^{*} \mathbf{d}\left\langle u_{1} \oplus \tau_{1}, u_{2} \oplus \tau_{2}\right\rangle_{\mathbb{B}} \\
= & \mathcal{D}_{\mathbb{B}}\left\langle u_{1} \oplus \tau_{1}, u_{2} \oplus \tau_{2}\right\rangle_{\mathbb{B}} .
\end{aligned}
$$


Next we prove (CA2). We have, using (21) to replace $\llbracket \tau_{1}, \tau_{2} \rrbracket Q^{*}$ by $-\Delta_{\partial_{Q} \tau_{2}} \tau_{1}+$ $\nabla_{\partial_{B} \tau_{1}} \tau_{2}+\rho_{Q}^{*} \mathbf{d}\left\langle\tau_{1}, \partial_{Q} \tau_{2}\right\rangle:$

$\left\langle\llbracket u_{1} \oplus \tau_{1}, u_{2} \oplus \tau_{2} \rrbracket_{\mathbb{B}}, u_{3} \oplus \tau_{3}\right\rangle_{\mathbb{B}}$

$=\left\langle\llbracket u_{1}, u_{2} \rrbracket_{U}+\nabla_{\partial_{B} \tau_{1}} u_{2}-\nabla_{\partial_{B}} \tau_{2} u_{1}, \tau_{3}\right\rangle$

$+\left\langle-\Delta_{\partial_{Q} \tau_{2}} \tau_{1}+\nabla_{\partial_{B} \tau_{1}}^{*} \tau_{2}+\rho_{Q}^{*} \mathbf{d}\left\langle\tau_{1}, \partial_{Q} \tau_{2}\right\rangle+\Delta_{u_{1}} \tau_{2}-\Delta_{u_{2}} \tau_{1}+\rho_{Q}^{*} \mathbf{d}\left\langle\tau_{1}, u_{2}\right\rangle, u_{3}\right.$

$\left.+\partial_{Q} \tau_{3}\right\rangle$.

We sum $\left\langle\llbracket u_{1} \oplus \tau_{1}, u_{2} \oplus \tau_{2} \rrbracket_{\mathbb{B}}, u_{3} \oplus \tau_{3}\right\rangle_{\mathbb{B}}$ with $\left\langle u_{2} \oplus \tau_{2}, \llbracket u_{1} \oplus \tau_{1}, u_{3} \oplus \tau_{3} \rrbracket_{\mathbb{B}}\right\rangle_{\mathbb{B}}$, and replace only in the first summand the term $\left\langle\Delta_{u_{1}} \tau_{2}, \partial_{Q} \tau_{3}\right\rangle$ by $\rho_{Q}\left(u_{1}\right)\left\langle\tau_{2}, \partial_{Q} \tau_{3}\right\rangle-$ $\left\langle\tau_{2}, \llbracket u_{1}, \partial_{Q} \tau_{3} \rrbracket\right\rangle$. This yields

$\left\langle\llbracket u_{1} \oplus \tau_{1}, u_{2} \oplus \tau_{2} \rrbracket_{\mathbb{B}}, u_{3} \oplus \tau_{3}\right\rangle_{\mathbb{B}}+\left\langle u_{2} \oplus \tau_{2}, \llbracket u_{1} \oplus \tau_{1}, u_{3} \oplus \tau_{3} \rrbracket_{\mathbb{B}}\right\rangle_{\mathbb{B}}$

$$
\begin{aligned}
= & \rho_{Q}\left(u_{1}\right)\left\langle u_{2}, \tau_{3}\right\rangle-\left\langle u_{2}, \Delta_{u_{1}} \tau_{3}\right\rangle+\left\langle\nabla_{\partial_{B} \tau_{1}} u_{2}-\nabla_{\partial_{B} \tau_{2}} u_{1}, \tau_{3}\right\rangle \\
& -\left\langle\Delta_{\partial} \tau_{2} \tau_{1}, u_{3}\right\rangle+\left\langle\nabla_{\partial_{B} \tau_{1}}^{*} \tau_{2}, u_{3}\right\rangle+\rho_{Q}\left(u_{3}\right)\left\langle\tau_{1}, \partial_{Q} \tau_{2}\right\rangle+\left\langle\Delta_{u_{1}} \tau_{2}, u_{3}\right\rangle-\left\langle\Delta_{u_{2}} \tau_{1}, u_{3}\right\rangle \\
& +\rho_{Q}\left(u_{3}\right)\left\langle\tau_{1}, u_{2}\right\rangle-\left\langle\Delta_{\partial_{Q} \tau_{2}} \tau_{1}, \partial_{Q} \tau_{3}\right\rangle+\left\langle\nabla_{\partial_{B}}^{*} \tau_{1}, \tau_{Q} \tau_{3}\right\rangle+\rho_{Q}\left(\partial_{Q} \tau_{3}\right)\left\langle\tau_{1}, \partial_{Q} \tau_{2}\right\rangle \\
& +\rho_{Q}\left(u_{1}\right)\left\langle\tau_{2}, \partial_{Q} \tau_{3}\right\rangle-\left\langle\tau_{2}, \llbracket u_{1}, \partial \tau_{3} \rrbracket\right\rangle-\left\langle\Delta_{u_{2}} \tau_{1}, \partial_{Q} \tau_{3}\right\rangle+\rho_{Q}\left(\partial_{Q} \tau_{3}\right)\left\langle\tau_{1}, u_{2}\right\rangle \\
& +\rho_{Q}\left(u_{1}\right)\left\langle u_{3}, \tau_{2}\right\rangle-\left\langle u_{3}, \Delta_{u_{1}} \tau_{2}\right\rangle+\left\langle\nabla_{\partial_{B}} \tau_{1} u_{3}-\nabla_{\partial_{B} \tau_{3}} u_{1}, \tau_{2}\right\rangle \\
& -\left\langle\Delta_{\partial_{Q} \tau_{3}} \tau_{1}, u_{2}\right\rangle+\left\langle\nabla_{\partial_{B} \tau_{1}}^{*} \tau_{3}, u_{2}\right\rangle+\rho_{Q}\left(u_{2}\right)\left\langle\tau_{1}, \partial_{Q} \tau_{3}\right\rangle+\left\langle\Delta_{u_{1}} \tau_{3}, u_{2}\right\rangle-\left\langle\Delta_{u_{3}} \tau_{1}, u_{2}\right\rangle \\
& +\rho_{Q}\left(u_{2}\right)\left\langle\tau_{1}, u_{3}\right\rangle-\left\langle\Delta_{\partial_{Q} \tau_{3}} \tau_{1}, \partial \tau_{2}\right\rangle+\left\langle\nabla_{\partial_{B}}^{*} \tau_{1}, \tau_{Q} \tau_{2}\right\rangle+\rho_{Q}\left(\partial_{Q} \tau_{2}\right)\left\langle\tau_{1}, \partial \tau_{3}\right\rangle \\
& +\left\langle\Delta_{u_{1}} \tau_{3}, \partial_{Q} \tau_{2}\right\rangle-\left\langle\Delta_{u_{3}} \tau_{1}, \partial_{Q} \tau_{2}\right\rangle+\rho_{Q}\left(\partial_{Q} \tau_{2}\right)\left\langle\tau_{1}, u_{3}\right\rangle .
\end{aligned}
$$

We reorder the remaining terms and replace eight times sums like $\rho_{Q}\left(\partial_{Q} \tau_{2}\right)\left\langle\tau_{1}, u_{3}\right\rangle-$ $\left\langle\Delta_{\partial_{Q} \tau_{2}} \tau_{1}, u_{3}\right\rangle$ by $\left\langle\llbracket \partial_{Q} \tau_{2}, u_{3} \rrbracket, \tau_{1}\right\rangle(2)$, and three times sums like $\left\langle\nabla_{\partial_{B}} \tau_{1} u_{2}, \tau_{3}\right\rangle+$ $\left\langle u_{2}, \nabla_{\partial_{B}}^{*} \tau_{1} \tau_{3}\right\rangle$ by $\rho_{B}\left(\partial_{B} \tau_{1}\right)\left\langle u_{2}, \tau_{3}\right\rangle$. This leads to

$$
\begin{aligned}
& \left\langle\llbracket u_{1} \oplus \tau_{1}, u_{2} \oplus \tau_{2} \rrbracket, u_{3} \oplus \tau_{3}\right\rangle_{\mathbb{B}}+\left\langle u_{2} \oplus \tau_{2}, \llbracket u_{1} \oplus \tau_{1}, u_{3} \oplus \tau_{3} \rrbracket\right\rangle_{\mathbb{B}} \\
& =\rho_{Q}\left(u_{1}\right)\left\langle u_{2} \oplus \tau_{2}, u_{3} \oplus \tau_{3}\right\rangle_{\mathbb{B}} \\
& +\left\langle\llbracket \partial_{Q} \tau_{2}, \partial_{Q} \tau_{3} \rrbracket, \tau_{1}\right\rangle+\left\langle\llbracket \partial_{Q} \tau_{2}, u_{3} \rrbracket, \tau_{1}\right\rangle+\left\langle\llbracket u_{3}, \partial_{Q} \tau_{2} \rrbracket, \tau_{1}\right\rangle+\left\langle\llbracket u_{2}, u_{3} \rrbracket, \tau_{1}\right\rangle \\
& +\left\langle\llbracket u_{3}, u_{2} \rrbracket, \tau_{1}\right\rangle+\left\langle\llbracket \partial_{Q} \tau_{3}, u_{2} \rrbracket, \tau_{1}\right\rangle+\left\langle\llbracket \llbracket u_{2}, \partial_{Q} \tau_{3} \rrbracket, \tau_{1}\right\rangle+\left\langle\llbracket \partial_{Q} \tau_{3}, \partial_{Q} \tau_{2} \rrbracket, \tau_{1}\right\rangle \\
& +\rho_{B}\left(\partial_{B} \tau_{1}\right)\left\langle u_{2}, \tau_{3}\right\rangle+\rho_{B}\left(\partial_{B} \tau_{1}\right)\left\langle\tau_{3}, \partial_{Q} \tau_{2}\right\rangle+\rho_{B}\left(\partial_{B} \tau_{1}\right)\left\langle u_{3}, \tau_{2}\right\rangle \\
& -\left\langle\nabla_{\partial_{B} \tau_{2}} u_{1}, \tau_{3}\right\rangle-\left\langle\tau_{2}, \llbracket u_{1}, \partial_{Q} \tau_{3} \rrbracket\right\rangle-\left\langle\nabla_{\partial_{B} \tau_{3}} u_{1}, \tau_{2}\right\rangle+\left\langle\Delta_{u_{1}} \tau_{3}, \partial_{Q} \tau_{2}\right\rangle .
\end{aligned}
$$

The four last terms cancel each other by (M1) and $\partial_{Q}=\partial_{Q}^{*}$. This yields

$$
\begin{aligned}
& \left\langle\llbracket u_{1} \oplus \tau_{1}, u_{2} \oplus \tau_{2} \rrbracket, u_{3} \oplus \tau_{3}\right\rangle_{\mathbb{B}}+\left\langle u_{2} \oplus \tau_{2}, \llbracket u_{1} \oplus \tau_{1}, u_{3} \oplus \tau_{3} \rrbracket\right\rangle_{\mathbb{B}} \\
= & \left(\rho_{Q}\left(u_{1}\right)+\rho_{B} \partial_{B} \tau_{1}\right)\left\langle u_{2} \oplus \tau_{2}, u_{3} \oplus \tau_{3}\right\rangle_{\mathbb{B}}=\rho_{\mathbb{B}}\left(u_{1} \oplus \tau_{1}\right)\left\langle u_{2} \oplus \tau_{2}, u_{3} \oplus \tau_{3}\right\rangle_{\mathbb{B}} .
\end{aligned}
$$

Finally we check the Jacobi identity in Leibniz form (CA1). We will check that

$\mathrm{Jac}_{\llbracket \cdot, \cdot \rrbracket}\left(u_{1} \oplus \tau_{1}, u_{2} \oplus \tau_{2}, u_{3} \oplus \tau_{3}\right)=\left(-\partial_{Q} v\right) \oplus v$

with $v=\left(R\left(\partial_{B} \tau_{1}, \partial_{B} \tau_{2}\right) u_{3}-\left\langle\mathbf{i}_{u_{2}} \mathbf{i}_{u_{1}} \omega, \partial_{B} \tau_{3}\right\rangle\right)+$ cyclic permutations. Since by Proposition $5.9 \mathbf{i}_{u_{2}} \mathbf{i}_{u_{1}} \omega$ has image in $U^{\circ}$ for all $u_{1}, u_{2} \in \Gamma(U)$ and $R\left(b_{1}, b_{2}\right)$ restricts 
to a morphism $U \rightarrow U^{\circ}$ for all $b_{1}, b_{2} \in \Gamma(B)$ by Proposition 5.5, $v$ is a section of

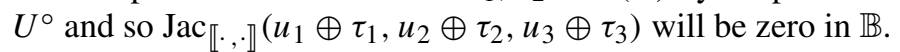

Using (29), (3), (39) and (11), we find

$\llbracket \llbracket u_{1} \oplus \tau_{1}, u_{2} \oplus \tau_{2} \rrbracket, u_{3} \oplus \tau_{3} \rrbracket$

$=\left(\llbracket \llbracket u_{1}, u_{2} \rrbracket_{U}, u_{3} \rrbracket_{U}+\llbracket \nabla_{\partial_{B} \tau_{1}} u_{2}-\nabla_{\partial_{B} \tau_{2}} u_{1}, u_{3} \rrbracket_{U}\right.$

$\left.+\nabla_{\left[\partial_{B} \tau_{1}, \partial_{B} \tau_{2}\right]+\partial_{B}\left(\Delta_{u_{1}} \tau_{2}-\Delta_{u_{2}} \tau_{1}\right)} u_{3}-\nabla_{\partial_{B} \tau_{3}}\left(\llbracket u_{1}, u_{2} \rrbracket_{U}+\nabla_{\partial_{B} \tau_{1}} u_{2}-\nabla_{\partial_{B} \tau_{2}} u_{1}\right)\right)$

$\oplus\left(\llbracket \llbracket \tau_{1}, \tau_{2} \rrbracket Q^{*}, \tau_{3} \rrbracket Q^{*}+\llbracket \Delta_{u_{1}} \tau_{2}-\Delta_{u_{2}} \tau_{1}, \tau_{3} \rrbracket Q^{*}\right.$

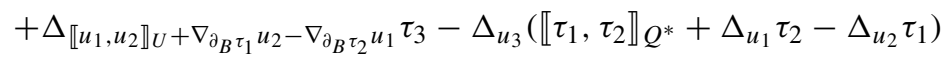

$\left.+\rho_{Q}^{*} \mathbf{d}\left\langle\llbracket \tau_{1}, \tau_{2} \rrbracket Q^{*}+\Delta_{u_{1}} \tau_{2}-\Delta_{u_{2}} \tau_{1}, u_{3}\right\rangle\right)$.

In the same manner, we compute

$\llbracket u_{1} \oplus \tau_{1}, \llbracket u_{2} \oplus \tau_{2}, u_{3} \oplus \tau_{3} \rrbracket \rrbracket$

$=\llbracket u_{1} \oplus \tau_{1},\left(\llbracket u_{2}, u_{3} \rrbracket_{U}+\nabla_{\partial_{B} \tau_{2}} u_{3}-\nabla_{\partial_{B} \tau_{3}} u_{2}\right) \oplus\left(\llbracket \tau_{2}, \tau_{3} \rrbracket Q^{*}\right.$

$\left.+\Delta_{u_{2}} \tau_{3}-\Delta_{u_{3}} \tau_{2}+\rho_{Q}^{*} \mathbf{d}\left\langle\tau_{2}, u_{3}\right\rangle\right) \rrbracket$

$=\left(\llbracket u_{1}, \llbracket u_{2}, u_{3} \rrbracket_{U} \rrbracket_{U}+\llbracket u_{1}, \nabla_{\partial_{B} \tau_{2}} u_{3}-\nabla_{\partial_{B} \tau_{3}} u_{2} \rrbracket_{U}\right.$

$\left.+\nabla_{\partial_{B} \tau_{1}}\left(\llbracket u_{2}, u_{3} \rrbracket_{U}+\nabla_{\partial_{B} \tau_{2}} u_{3}-\nabla_{\partial_{B} \tau_{3}} u_{2}\right)-\nabla_{\left[\partial_{B} \tau_{2}, \partial_{B} \tau_{3}\right]+\partial_{B}\left(\Delta_{u_{2}} \tau_{3}-\Delta_{u_{3}} \tau_{2}\right)} u_{1}\right)$

$\oplus\left(\llbracket \tau_{1}, \llbracket \tau_{2}, \tau_{3} \rrbracket Q^{*} \rrbracket Q^{*}+\llbracket \tau_{1}, \Delta_{u_{2}} \tau_{3}-\Delta_{u_{3}} \tau_{2} \rrbracket Q^{*}+\rho_{Q}^{*} \mathbf{d}\left(\rho_{Q}\left(\partial_{Q} \tau_{1}\right)\left\langle\tau_{2}, u_{3}\right\rangle\right)\right.$

$+\Delta_{u_{1}}\left(\llbracket \tau_{2}, \tau_{3} \rrbracket Q^{*}+\Delta_{u_{2}} \tau_{3}-\Delta_{u_{3}} \tau_{2}\right)+\rho_{Q}^{*} \mathbf{d}\left(\rho_{Q}\left(u_{1}\right)\left\langle\tau_{2}, u_{3}\right\rangle\right)$

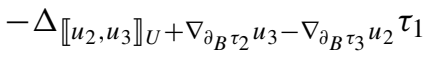

$$
\left.+\rho_{Q}^{*} \mathbf{d}\left\langle\tau_{1}, \llbracket u_{2}, u_{3} \rrbracket_{U}+\nabla_{\partial_{B} \tau_{2}} u_{3}-\nabla_{\partial_{B} \tau_{3}} u_{2}\right\rangle\right)
$$

Since $\mathrm{Jac}_{\llbracket \cdot, \cdot \rrbracket_{U}}\left(u_{1}, u_{2}, u_{3}\right)=0$ and $\partial_{B} \circ \Delta_{q}=\nabla_{q} \circ \partial_{B}$ for all $q \in \Gamma(Q)$, the $U$-term of $\mathrm{Jac}_{\llbracket,, \rrbracket}\left(u_{1} \oplus \tau_{1}, u_{2} \oplus \tau_{2}, u_{3} \oplus \tau_{3}\right)$ equals

$$
\begin{aligned}
& \llbracket \nabla_{\partial_{B} \tau_{1}} u_{2}-\nabla_{\partial_{B} \tau_{2}} u_{1}, u_{3} \rrbracket_{U}
\end{aligned}
$$

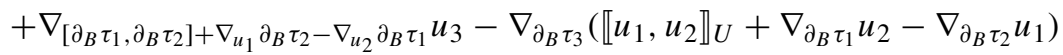

$$
\begin{aligned}
& +\llbracket u_{2}, \nabla_{\partial_{B} \tau_{1}} u_{3}-\nabla_{\partial_{B} \tau_{3}} u_{1} \rrbracket_{U}
\end{aligned}
$$

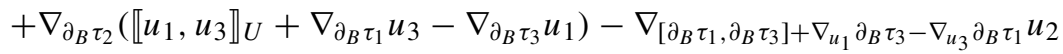

$$
\begin{aligned}
& -\llbracket u_{1}, \nabla_{\partial_{B} \tau_{2}} u_{3}-\nabla_{\partial_{B} \tau_{3}} u_{2} \rrbracket_{U} \\
& -\nabla_{\partial_{B} \tau_{1}}\left(\llbracket u_{2}, u_{3} \rrbracket_{U}+\nabla_{\partial_{B} \tau_{2}} u_{3}-\nabla_{\partial_{B} \tau_{3}} u_{2}\right)+\nabla_{\left[\partial_{B} \tau_{2}, \partial_{B} \tau_{3}\right]+\nabla_{u_{2}} \partial_{B} \tau_{3}-\nabla_{u_{3}} \partial_{B} \tau_{2} u_{1}} .
\end{aligned}
$$

Note that since for any $b_{1}, b_{2} \in \Gamma(B), R\left(b_{1}, b_{2}\right)$ restricts to a section of $\operatorname{Hom}\left(U, U^{\circ}\right)$ (see Section 5.2), the last summand on the right hand side of (M4) vanishes on sections of $U$. By sorting out the terms and using (M4) on sections of $U$, we get

$$
\begin{aligned}
& -R_{\nabla}\left(\partial_{B} \tau_{3}, \partial_{B} \tau_{1}\right) u_{2}-R_{\nabla}\left(\partial_{B} \tau_{1}, \partial_{B} \tau_{2}\right) u_{3}-R_{\nabla}\left(\partial_{B} \tau_{2}, \partial_{B} \tau_{3}\right) u_{1} \\
& +\partial_{Q}\left\langle\mathbf{i}_{u_{2}} \mathbf{i}_{u_{1}} \omega, \partial_{B} \tau_{3}\right\rangle+\partial_{Q}\left\langle\mathbf{i}_{u_{3}} \mathbf{i}_{u_{2}} \omega, \partial_{B} \tau_{1}\right\rangle+\partial_{Q}\left\langle\mathbf{i}_{u_{1}} \mathbf{i}_{u_{3}} \omega, \partial_{B} \tau_{2}\right\rangle .
\end{aligned}
$$


Since $R_{\nabla}=\partial_{Q} \circ R$, this is $-\partial_{Q} v$. We conclude by computing the $Q^{*}$-part of $\mathrm{Jac}_{\llbracket \cdot, \cdot \rrbracket}\left(u_{1} \oplus \tau_{1}, u_{2} \oplus \tau_{2}, u_{3} \oplus \tau_{3}\right)$. Again, because $\mathrm{Jac}_{\llbracket \cdot, \cdot \rrbracket_{Q^{*}}}\left(\tau_{1}, \tau_{2}, \tau_{3}\right)=0$, we get

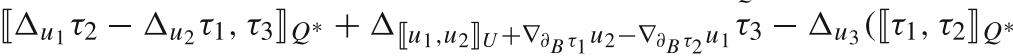

$$
\begin{aligned}
& \left.+\Delta_{u_{1}} \tau_{2}-\Delta_{u_{2}} \tau_{1}\right) \\
& +\rho_{Q}^{*} \mathbf{d}\left\langle\llbracket \tau_{1}, \tau_{2} \rrbracket Q^{*}+\Delta_{\pi_{1} \tau_{2}}-\Delta_{u_{2} \tau_{1}}, u_{3}\right\rangle+\llbracket \tau_{2}, \Delta_{u_{1}} \tau_{3}-\Delta_{u_{3}} \tau_{1} \rrbracket Q^{*} \\
& +\rho_{Q}^{*} \mathbf{d}\left(\rho_{Q}\left(\partial_{Q} \tau_{2}\right)\left\langle\tau_{1}, u_{3}\right\rangle\right) \\
& +\Delta_{u_{2}}\left(\llbracket \tau_{1}, \tau_{3} \rrbracket Q^{*}+\Delta_{u_{1}} \tau_{3}-\Delta_{u_{3}} \tau_{1}\right)+\rho_{Q}^{*} \mathbf{d}\left(\rho_{Q}\left(u_{2}\right)\left(\tau_{1}, u_{3}\right\rangle\right)
\end{aligned}
$$

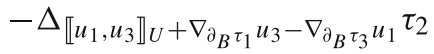

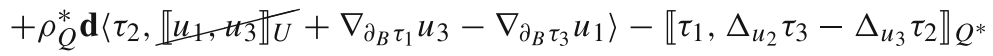

$$
\begin{aligned}
& -\rho_{Q}^{*} \mathbf{d}\left(\rho_{Q}\left(\partial_{Q} \tau_{1}\right)\left\langle\tau_{2}, u_{3}\right\rangle\right) \\
& -\Delta_{u_{1}}\left(\llbracket \tau_{2}, \tau_{3} \rrbracket_{Q^{*}}+\Delta_{u_{2}} \tau_{3}-\Delta_{u_{3}} \tau_{2}\right)-\rho_{Q}^{*} \mathbf{d}\left(\rho_{Q}\left(u_{1}\right)\left\langle\tau_{2}, u_{3}\right\rangle\right) \\
& +\Delta_{\llbracket u_{2}, u_{3} \rrbracket_{U}+\nabla_{\partial_{B}} \tau_{2} u_{3}-\nabla_{\partial_{B}} \tau_{3} u_{2}} \tau_{1}
\end{aligned}
$$

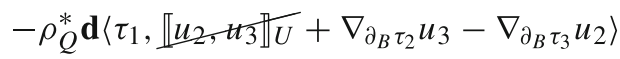

The six cancelling terms cancel by (2). Reordering the terms, we get using Lemma A.1:

$$
\begin{aligned}
& -R_{\Delta}\left(u_{3}, u_{1}\right) \tau_{2}-R_{\Delta}\left(u_{2}, u_{3}\right) \tau_{1}-R_{\Delta}\left(u_{1}, u_{2}\right) \tau_{3} \\
& +R\left(\partial_{B} \tau_{2}, \partial_{B} \tau_{3}\right) u_{1}+R\left(\partial_{B} \tau_{1}, \partial_{B} \tau_{2}\right) u_{3}-R\left(\partial_{B} \tau_{1}, \partial_{B} \tau_{3}\right) u_{2} \\
& -\rho_{Q}^{*} \mathbf{d}\left\langle\tau_{2}, \partial_{Q} \Delta_{u_{3}} \tau_{1}\right\rangle+\rho_{Q}^{*} \mathbf{d}\left\langle\llbracket \tau_{1}, \tau_{2} \rrbracket Q^{*}, u_{3}\right\rangle+\rho_{Q}^{*} \mathbf{d}\left(\rho_{Q}\left(\partial_{Q} \tau_{2}\right)\left\langle\tau_{1}, u_{3}\right\rangle\right) \\
& +\rho_{Q}^{*} \mathbf{d}\left\langle\tau_{2}, \nabla_{\partial_{B} \tau_{1}} u_{3}\right\rangle-\rho_{Q}^{*} \mathbf{d}\left(\rho_{Q}\left(\partial_{Q} \tau_{1}\right)\left\langle\tau_{2}, u_{3}\right\rangle\right) .
\end{aligned}
$$

For the second use of Lemma A.1, we had to replace $-\llbracket \tau_{2}, \Delta_{u_{3}} \tau_{1} \rrbracket Q^{*}$ by $\llbracket \Delta_{u_{3}} \tau_{1}, \tau_{2} \rrbracket Q^{*}-\rho_{Q}^{*} \mathbf{d}\left\langle\tau_{2}, \partial_{Q} \Delta_{u_{3}} \tau_{1}\right\rangle$. This is why we get the first term on the third line. Using (13), we get $v+\rho_{Q}^{*} \mathbf{d} f$ with $f \in C^{\infty}(M)$ defined by

$$
\begin{aligned}
f= & -\left\langle\tau_{2}, \partial_{Q} \Delta_{u_{3}} \tau_{1}\right\rangle+\left\langle\Delta_{\partial_{Q} \tau_{1}} \tau_{2}-\nabla_{\partial_{B} \tau_{2}}^{*} \tau_{1}, u_{3}\right\rangle+\rho_{Q}\left(\partial_{Q} \tau_{2}\right)\left\langle\tau_{1}, u_{3}\right\rangle \\
& +\left\langle\tau_{2}, \nabla_{\partial_{B} \tau_{1}} u_{3}\right\rangle-\rho_{Q}\left(\partial_{Q} \tau_{1}\right)\left\langle\tau_{2}, u_{3}\right\rangle \\
= & \left\langle\tau_{2},-\partial_{Q} \Delta_{u_{3}} \tau_{1}-\llbracket \partial_{Q} \tau_{1}, u_{3} \rrbracket+\nabla_{\partial_{B} \tau_{1}} u_{3}\right\rangle+\rho_{Q}\left(\partial_{Q} \tau_{2}\right)\left\langle\tau_{1}, u_{3}\right\rangle-\left\langle\nabla_{\partial_{B} \tau_{2}}^{*} \tau_{1}, u_{3}\right\rangle .
\end{aligned}
$$

By (M1), the first pairing equals $-\left\langle\tau_{1}, \nabla_{\partial_{B}} \tau_{2} u_{3}\right\rangle$. Hence, we find $f=0$ using $\rho_{B} \circ$ $\partial_{B}=\rho_{Q} \circ \partial_{Q}$. We have proved $\mathrm{Jac}_{\llbracket \cdot, \cdot \rrbracket}\left(u_{1} \oplus \tau_{1}, u_{2} \oplus \tau_{2}, u_{3} \oplus \tau_{3}\right)=\left(-\partial_{Q} v\right) \oplus v$.

\section{References}

1. Courant, T.J., Weinstein, A.: Beyond Poisson structures. In: Action Hamiltoniennes de Groupes. Troisième Théorème de Lie (Lyon, 1986), volume 27 of Travaux en Cours, pp. 39-49. Hermann, Paris (1988)

2. del Carpio-Marek, F.: Geometric structures on degree 2 manifolds. PhD thesis, IMPA, available at www.impa.br/wp-content/uploads/2017/05/Fernando_Del_Carpio.pdf Rio de Janeiro (2015)

3. Drummond, T., Jotz Lean, M., Ortiz, C.: VB-algebroid morphisms and representations up to homotopy. Differ. Geom. Appl. 40, 332-357 (2015)

4. Gracia-Saz, A., Mehta, R.A.: Lie algebroid structures on double vector bundles and representation theory of Lie algebroids. Adv. Math. 223(4), 1236-1275 (2010) 
5. Gracia-Saz, A., Jotz Lean, M., Mackenzie, K.C.H., Mehta, R.A.: Double Lie algebroids and representations up to homotopy. J. Homotopy Relat. Struct. 13(2), 287-319 (2018)

6. Gualtieri, M.: Generalized complex geometry. PhD thesis (2003)

7. Hitchin, N.: Generalized Calabi-Yau manifolds. Q. J. Math. 54(3), 281-308 (2003)

8. Jotz Lean, M.: $\mathbb{N}$-manifolds of degree 2 and metric double vector bundles. arXiv:1504.00880 (2015)

9. Jotz Lean, M.: Dorfman connections and Courant algebroids. J. Math. Pures Appl. 116(9), 1-39 (2018)

10. Jotz Lean, M.: The geometrization of $\mathbb{N}$-manifolds of degree 2. J. Geom. Phys. 133, 113-140 (2018)

11. Jotz Lean, M.: Dirac groupoids and Dirac bialgebroids. J. Symplectic Geom. 17(1), 179-238 (2019)

12. Jotz Lean, M.: Lie 2-algebroids and matched pairs of 2-representations-a geometric approach. Pac. J. Math. 301(1), 143-188 (2019)

13. Jotz Lean, M., Mehta, R.A., Papantonis, T.: Modules and representations up to homotopy of Lie $n$-algebroids. page arXiv:2001.01101 (2020)

14. Li-Bland, D.: Phd thesis: LA-Courant Algebroids and their Applications. arXiv:1204.2796 (2012)

15. Li-Bland, D.: Pseudo-Dirac structures. Indag. Math. (N.S.) 25(5), 1054-1101 (2014)

16. Li-Bland, D., Ševera, P.: Quasi-Hamiltonian groupoids and multiplicative Manin pairs. Int. Math. Res. Not. IMRN 10, 2295-2350 (2011)

17. Liu, Z.-J., Weinstein, A., Xu, P.: Manin triples for Lie bialgebroids. J Differ. Geom. 45(3), 547-574 (1997)

18. Mackenzie, K.C.H.: Duality and triple structures. In: The breadth of symplectic and Poisson geometry, volume 232 of Progr. Math., pp. 455-481. Birkhäuser, Boston (2005)

19. Mackenzie, K.C.H.: General Theory of Lie Groupoids and Lie Algebroids, volume 213 of London Mathematical Society Lecture Note Series. Cambridge University Press, Cambridge (2005)

20. Mackenzie, K.C.H.: Ehresmann doubles and Drinfel'd doubles for Lie algebroids and Lie bialgebroids. J. Reine Angew. Math. 658, 193-245 (2011)

21. Mehta, R.A., Tang, X.: From double Lie groupoids to local Lie 2-groupoids. Bull. Braz. Math. Soc. (N.S.) 42(4), 651-681 (2011)

22. Mehta, R.A., Tang, X.: Symplectic structures on the integration of exact Courant algebroids. J. Geom. Phys. 127, 68-83 (2018)

23. Pradines, J.: Fibrés Vectoriels Doubles et Calcul des Jets non Holonomes, volume 29 of Esquisses Mathématiques [Mathematical Sketches]. Université d'Amiens U.E.R. de Mathématiques, Amiens (1977)

24. Roytenberg, D.: Courant algebroids, derived brackets and even symplectic supermanifolds. ProQuest LLC, Ann Arbor. Thesis (Ph.D.)-University of California, Berkeley (1999)

25. Roytenberg, D.: On the structure of graded symplectic supermanifolds and Courant algebroids. In: Quantization, Poisson Brackets and Beyond (Manchester, 2001), volume 315 of Contemp. Math., pp. 169-185. American Mathematical Society, Providence (2002)

26. Severa, P.: Some title containing the words "homotopy" and "symplectic", e.g. this one. In: Travaux Mathématiques. Fasc. XVI, Trav. Math., XVI, pp. 121-137. University of Luxembourg, Luxembourg (2005)

27. Ševera, P., Širaň, M.: Integration of differential graded manifolds. arXiv e-prints, page arXiv:1506.04898 (2015)

28. Sheng, Y., Zhu, C.: Higher extensions of Lie algebroids. Commun. Contemp. Math. 19(3), 1650034, 41 (2017)

29. Uchino, K.: Remarks on the definition of a Courant algebroid. Lett. Math. Phys. 60(2), 171-175 (2002)

Publisher's Note Springer Nature remains neutral with regard to jurisdictional claims in published maps and institutional affiliations. 